Aus der Klinik für Urologie

(Prof. Dr. med. L.Trojan)

der Medizinischen Fakultät der Universität Göttingen

\title{
Untersuchung des Stellenwerts von transurethraler und suprapubischer Harnableitung in der Therapie von Prostatitis, Epididymitis und Pyelonephritis
}

\author{
INAUGURAL-DISSERTATION \\ zur Erlangung des Doktorgrades \\ der Medizinischen Fakultät der \\ Georg-August-Universität zu Göttingen
}

vorgelegt von

Marlena Schubert geb. Stäblein

aus

Herzberg am Harz

Göttingen 2019 
Dekan:

\section{Betreuungsausschuss}

Betreuer/in:

Ko-Betreuer/in:

\section{Prüfungskommission}

Referent/in:

Ko-Referent/in:

Drittreferent/in:

Datum der mündlichen Prüfung: 12.02.2020
Prof. Dr. med. S. Ahyai

Prof. Dr. med. Dr. rer. nat. H. Eiffert

Prof. Dr. med. S. Ahyai

Prof. Dr. med. Dr. rer. nat. H. Eiffert

Prof. Dr. M. Schön 
Hiermit erkläre ich, die Dissertation mit dem Titel " Untersuchung des Stellenwerts von transurethraler und suprapubischer Harnableitung in der Therapie von Prostatitis, Epididymitis und Pyelonephritis" eigenständig angefertigt und keine anderen als die von mir angegebenen Quellen und Hilfsmittel verwendet zu haben.

Göttingen, den

(Unterschrift) 


\section{Inhaltsverzeichnis}

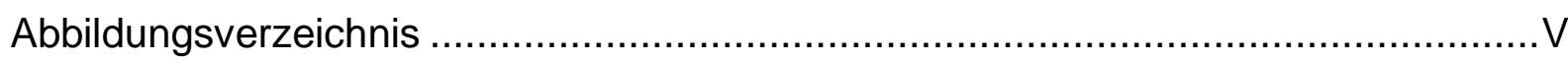

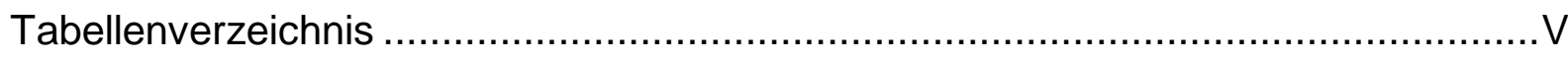

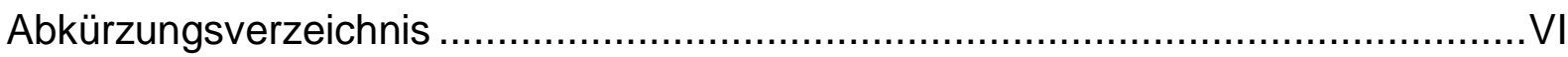

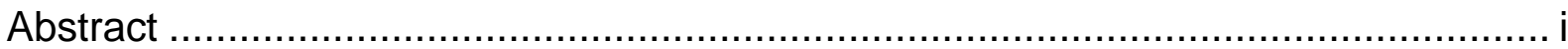

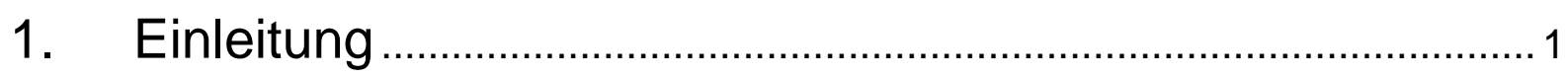

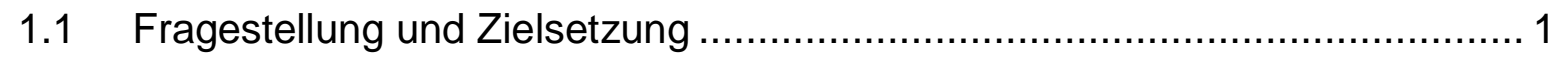

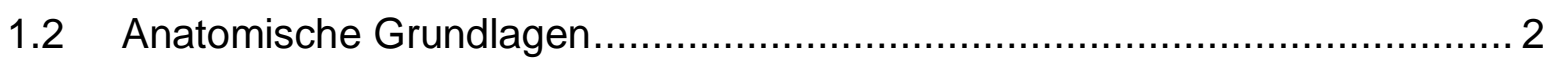

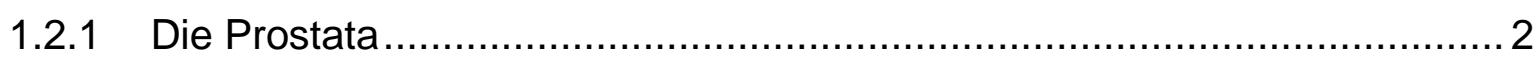

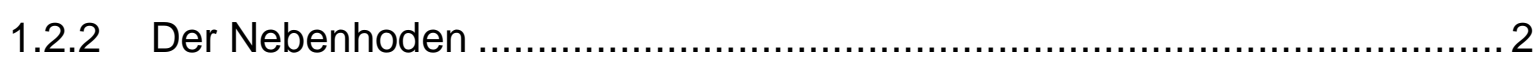

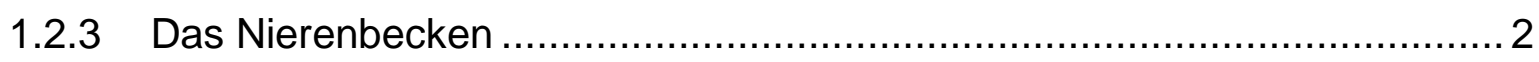

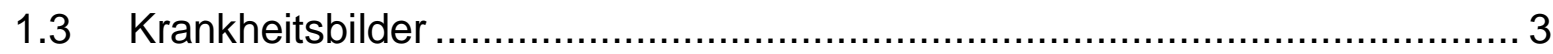

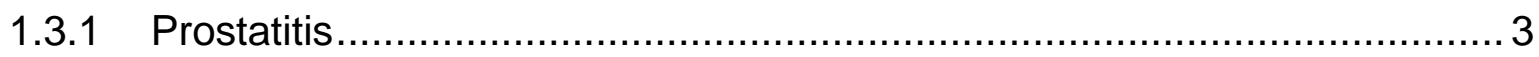

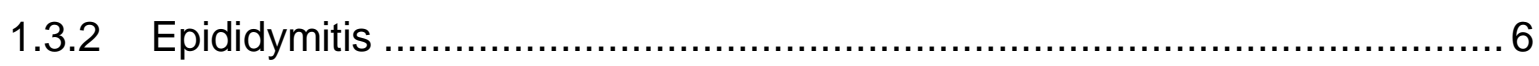

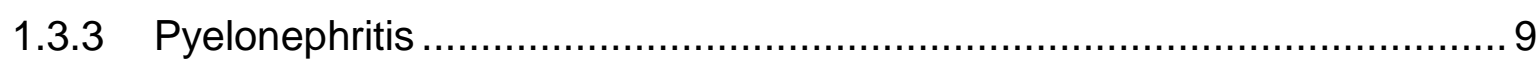

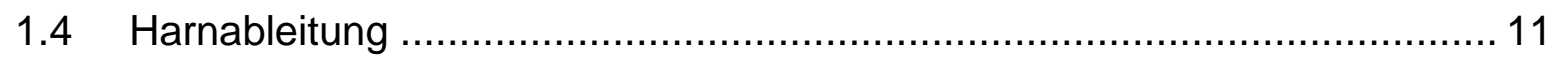

1.4.1 Transurethrale Harnableitung …………..................................... 12

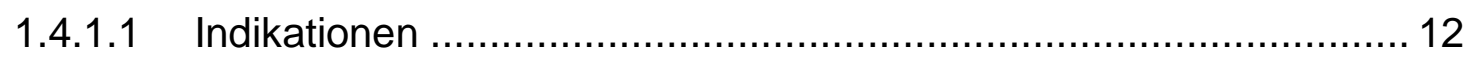

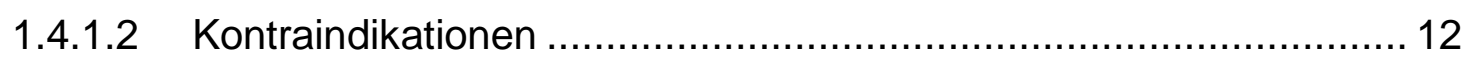

1.4.2 Suprapubische Harnableitung ....................................................... 13

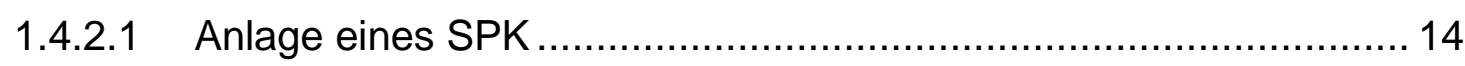

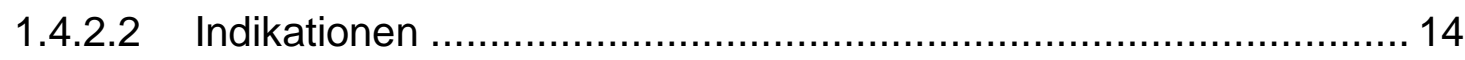

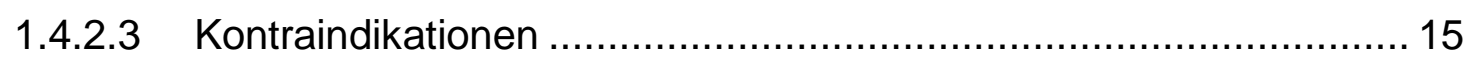

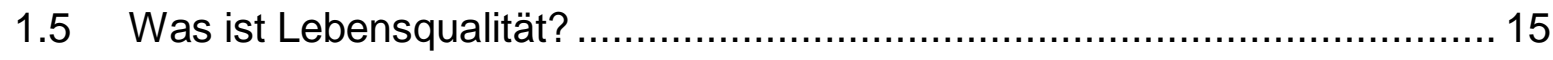

1.6 Verfahren zur Messung der Lebensqualität............................................. 16

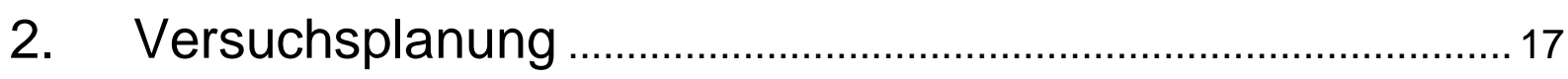

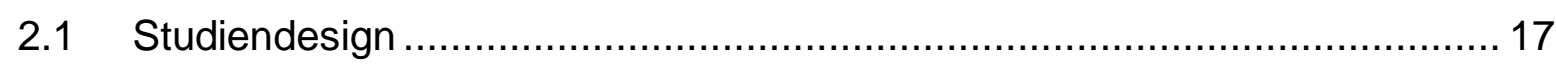

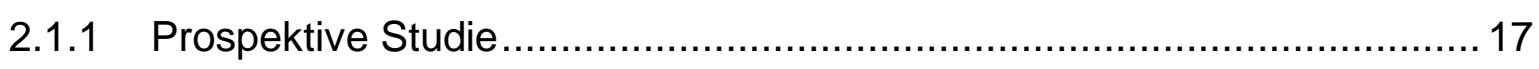

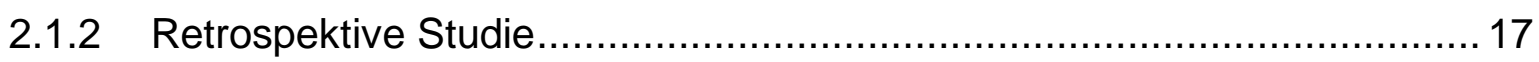

2.2 Studienvorbereitungen / Fallzahlabschätzung ....................................... 18 
3. Material und Methoden ................................................................ 19

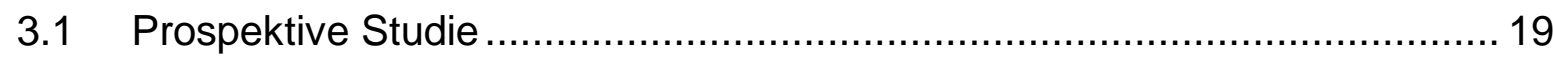

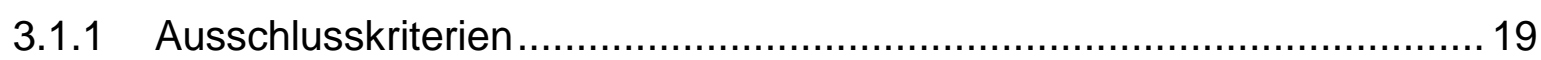

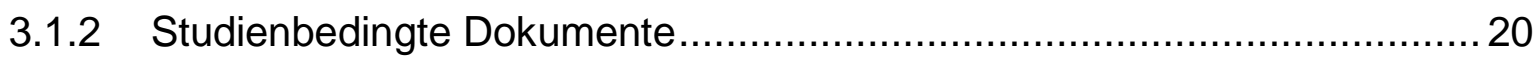

3.1.2.1 Der SF-12-Fragebogen .................................................... 20

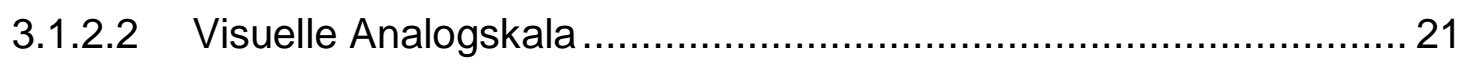

3.1.3 Studiendurchführung Männer mit Prostatitis/Epididymitis ........................2 21

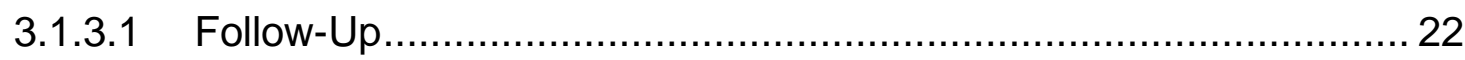

3.1.4 Studiendurchführung Frauen mit Pyelonephritis .................................. 24

3.1.5 Datenerfassung prospektive Studie ................................................ 26

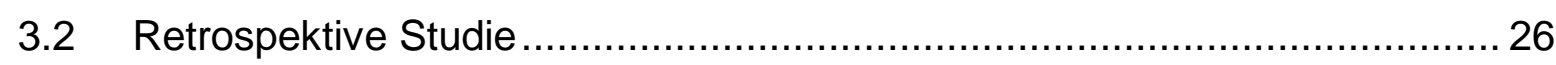

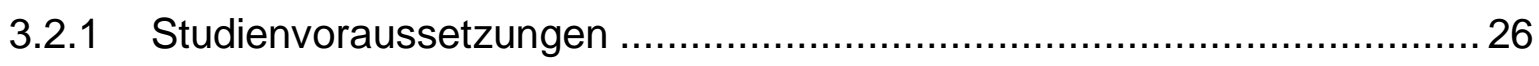

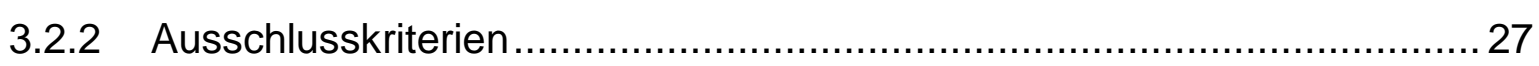

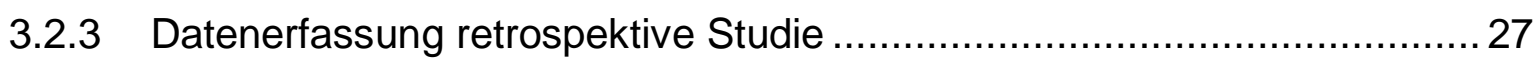

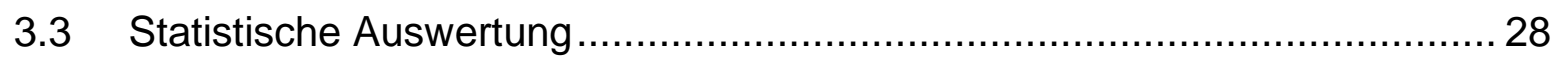

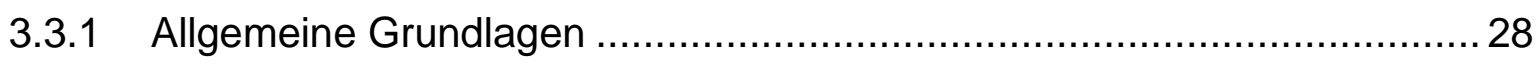

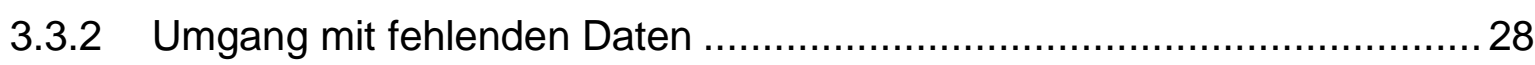

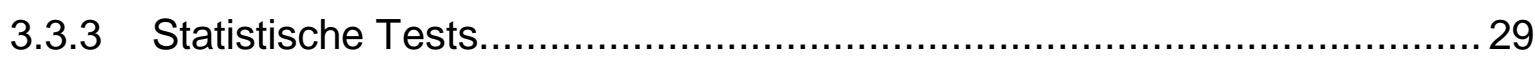

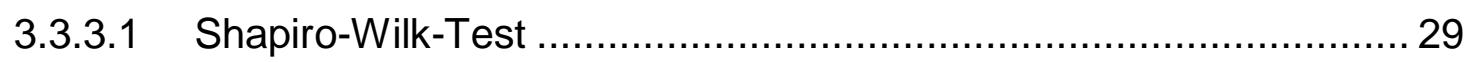

3.3.3.2 Wilcoxon-Rangsummentest ..................................................... 30

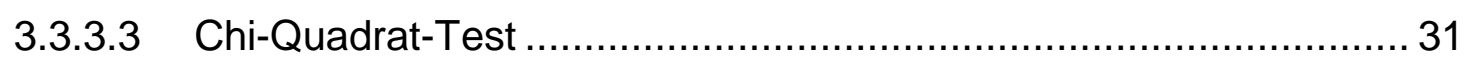

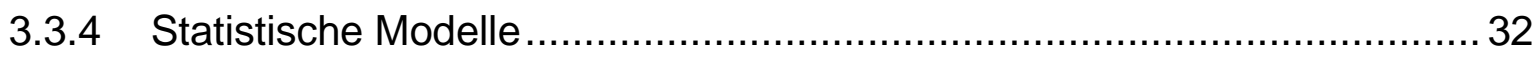

3.3.4.1 Lineare Regression ............................................................ 32

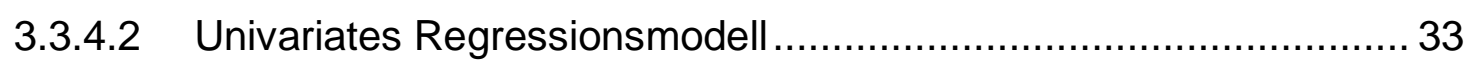

3.3.4.3 Multivariables Regressionsmodell ................................................... 34

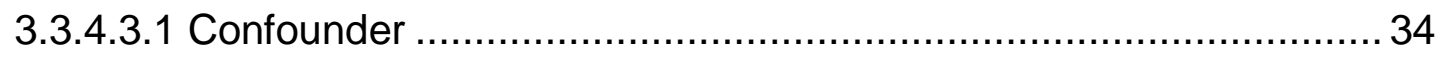

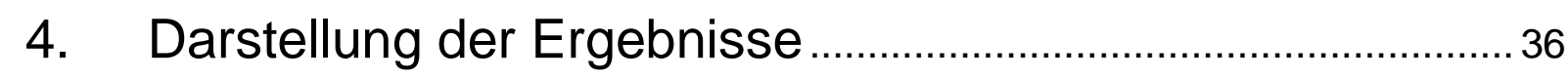

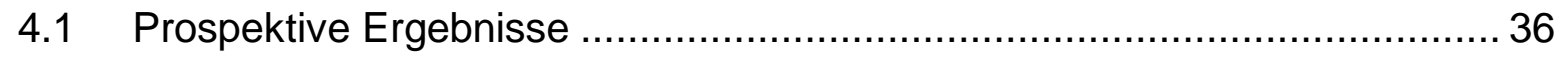

4.1.1 Übersicht Patientenkollektiv Männer .................................................. 36

4.1.1.1 Ergebnisse Qol-Fragebogen und VAS ..................................... 37

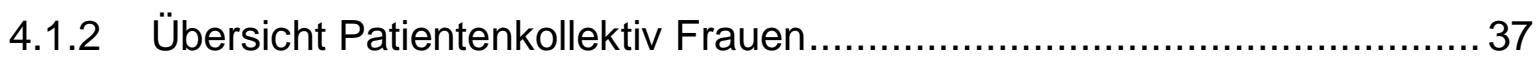

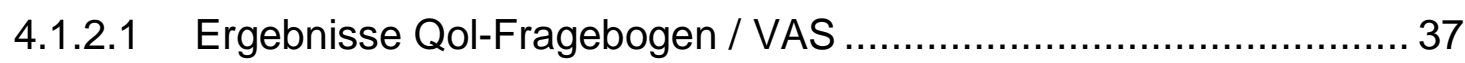




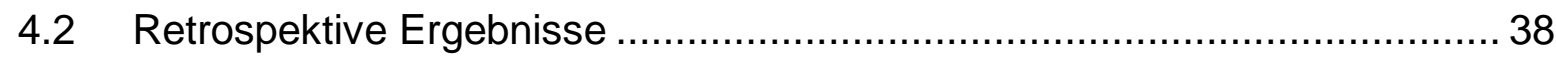

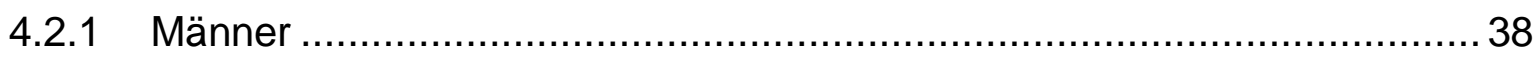

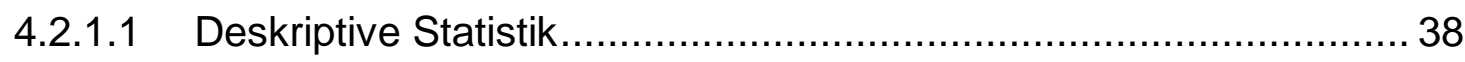

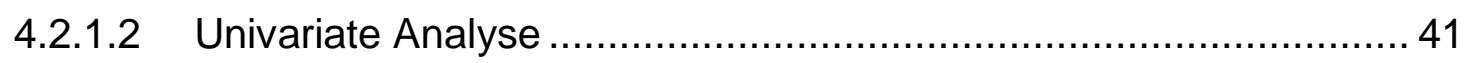

4.2.1.3 Multivariable Analyse .......................................................... 43

4.2.1.4 Outcome Krankenhausaufenthalt Männer > 63 Jahre $(n=19) \ldots \ldots . . .46$

4.2.1.5 Outcome Krankenhausaufenthalt Männer $\leq 63$ Jahre $(n=31) \ldots \ldots . . .47$

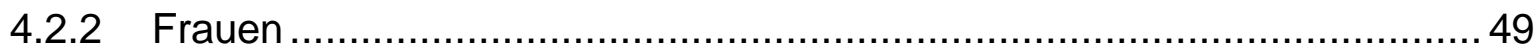

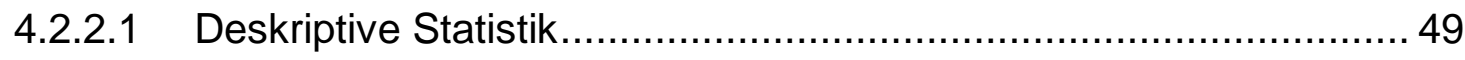

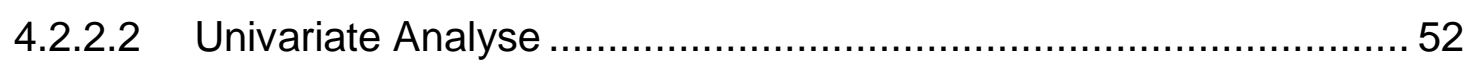

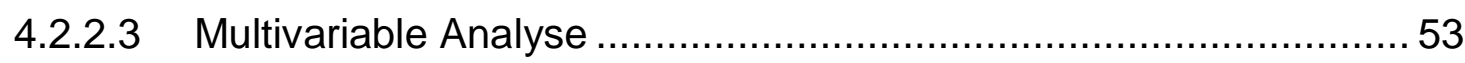

4.2.3 Gemeinsames Patientenkollektiv (Frauen + Männer) …….................... 55

4.2.4 Veränderte Interventionsvariablen ..................................................... 55

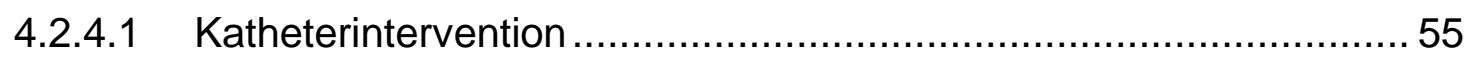

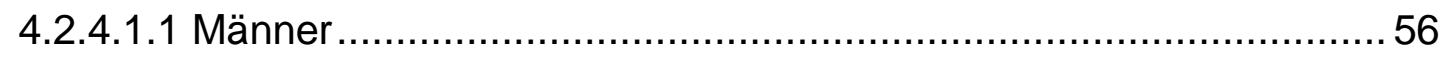

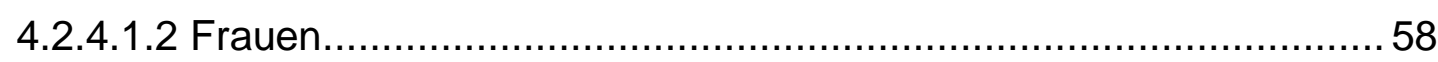

4.2.4.2 Ergebnisse klinischer Parameter ................................................. 60

4.2.4.3 Ergebnisse der Antibiotikatherapie ............................................... 64

4.2.4.4 Betrachtung des Charlson Comorbidity Index .............................. 64

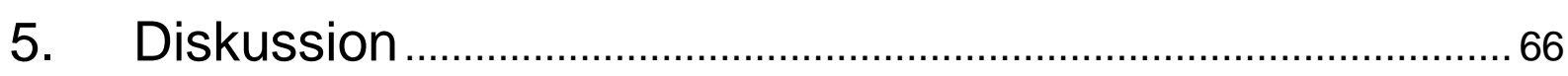

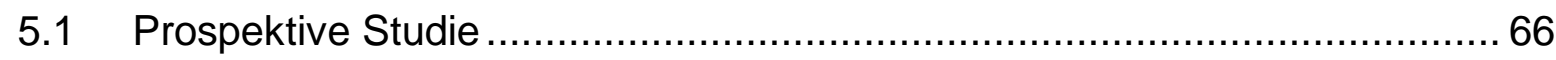

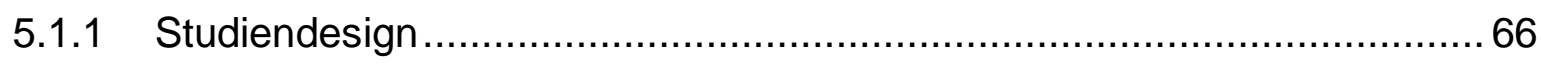

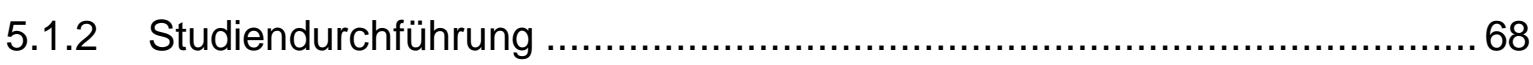

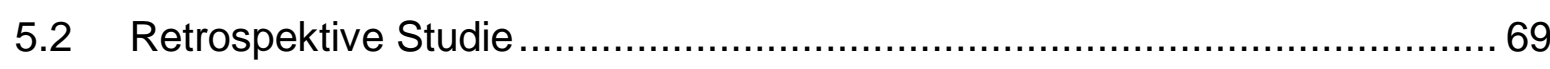

5.2.1 Diskussion der Ergebnisse Männer .................................................. 69

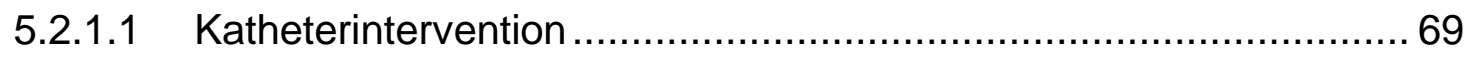

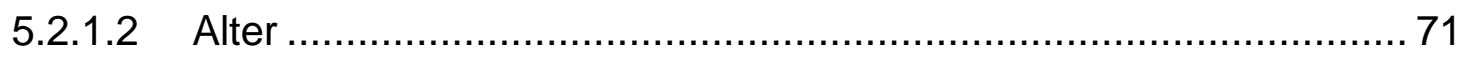

5.2.1.3 Antibiotikatherapie ......................................................... 72

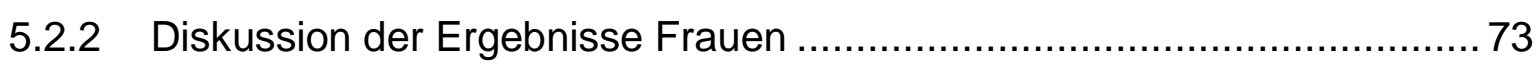

5.2.2.1 Katheterintervention .......................................................... 73

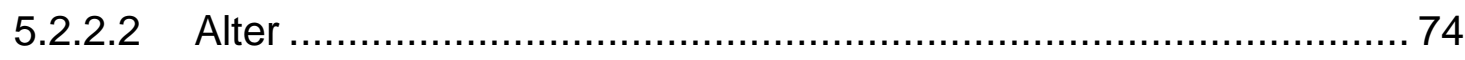

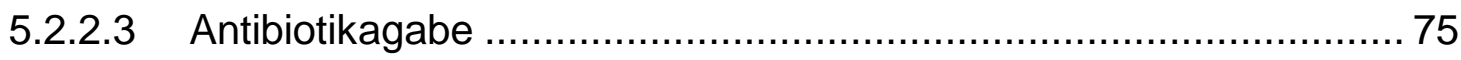




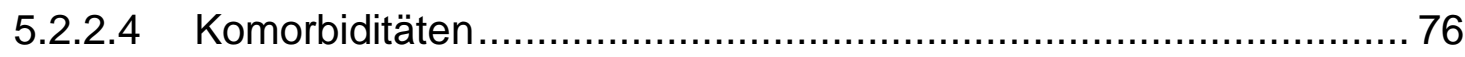

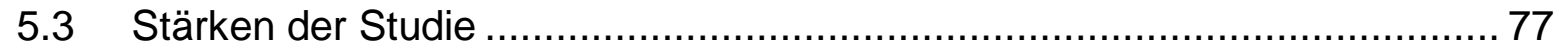

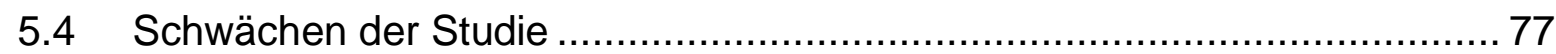

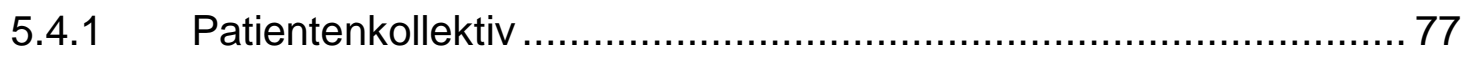

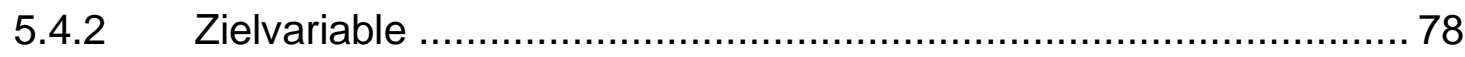

5.4.2.1 Zeitliche Erfassung der SPK-Liegedauer.................................... 78

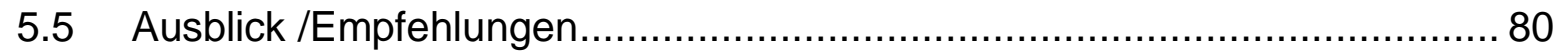

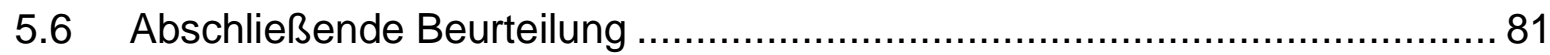

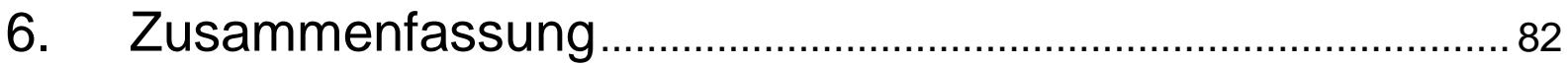

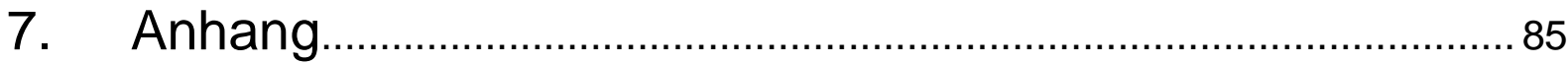

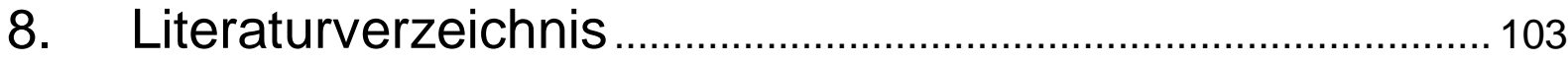




\section{Abbildungsverzeichnis}

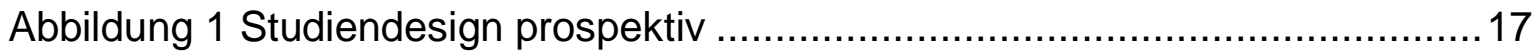

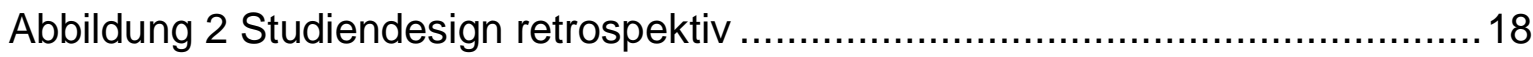

Abbildung 3 Beispiel einer Visuellen Analogskala ...........................................21

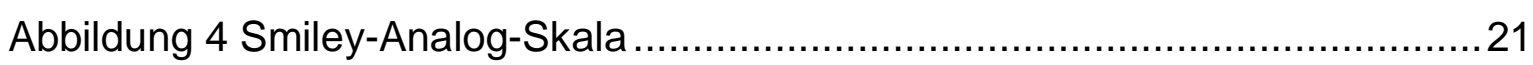

Abbildung 5 Ablaufschema prospektive Studie der Männer ...............................23

Abbildung 6 Ablaufschema prospektive Studie der Frauen ................................25

Abbildung 7 Beispiel grafische Darstellung einer linearen Regression ..................33

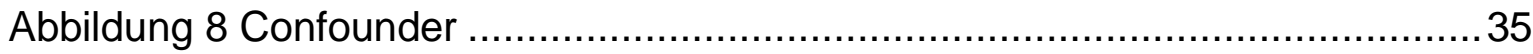

Abbildung 9 Verteilung der Kathetertypen im männlichen Kollektiv ......................56

Abbildung 10 Verteilung Liegedauer SPK und DK der Männer ...........................57

Abbildung 11 Krankenhausaufenthalt der Männer in Kathetergruppen .................58

Abbildung 12 Krankenhausaufenthalt der Frauen mit/ohne Katheter ...................59

Abbildung 13 Männliche CRP-Werte bei Aufnahme nach Alter .............................60

Abbildung 14 Leukozytenwerte Männer bei Aufnahme nach Alter ........................61

Abbildung 15 CRP-Werte der Frauen bei Aufnahme in Kathetergruppen ..............62

Abbildung 16 Leukozytenwerte der Frauen bei Aufnahme in Kathetergruppen ....63

\section{Tabellenverzeichnis}

Tabelle 1 Beispielhafte Berechnung des Wilcoxon-Rangsummentests ..................31

Tabelle 2 Männliches Patientenkollektiv der prospektiven Studie .........................36

Tabelle 3 Weibliches Patientenkollektiv der prospektiven Studie ......................... 37

Tabelle 4 Übersicht aller Parameter der männlichen Studienpatienten .................38

Tabelle 5 Univariate Analyse der Männer ......................................................... 42

Tabelle 6 Lineares multivariables Modell ohne Interaktionsterm ..........................44

Tabelle 7 Multivariable Analyse mit Interaktionsterm ............................................45

Tabelle 8 Subgruppenanalyse der Männer > 63 Jahre......................................46

Tabelle 9 Subgruppenanalyse der Männer $\leq 63$ Jahre ...................................... 48

Tabelle 10 Übersicht aller Parameter der weiblichen Studienpatienten .................49

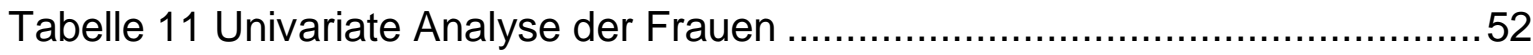

Tabelle 12 Ergebnisse Outcome Krankenhausaufenthalt der Frauen ..................54 


\section{Abkürzungsverzeichnis}

$A B$

BSG

CRP

DK

DRU

EAU

E. coli

GE-Aktivität

HWI

iv

$\mathrm{KI}$

LUTS

Mikrol

MSM

$\mathrm{NIH}$

NLGA

NSAR

PSA

Qol

Ref.

S. aureus

SD

SPK

UMG

VAS

WHO
Antibiotikum

Blutsenkungsgeschwindigkeit

C-reaktives Protein

Dauerkatheter (transurethral)

Digital-rektale Untersuchung

European Association of Urology

Escherichia Coli

Granulozytenesterase-Aktivität

Harnwegsinfekt

intravenös

Konfidenzintervall

Lower Urinary Tract Symptoms

Mikroliter

Men who have sex with men

National Institutes of Health

Niedersächsisches Gesundheitsamt

nichtsteroidale Antirheumatika

Prostata-spezifische Antigen

Quality of Life

Referenzkategorie

Staphylococcus aureus

Standardabweichung

suprapubischer Katheter

Universitätsmedizin Göttingen

Visuelle Analogskala

Weltgesundheitsorganisation 


\section{Abstract}

Im klinischen Alltag sind komplizierte Harnwegsinfektionen wie die Prostatitis und Epididymitis beim Mann oder die Pyelonephritis bei der Frau häufige Hospitalisierungsgründe urologischer Patienten. Sie bedeuten für den betroffenen Patienten nicht nur körperliche Beschwerden wie Schmerzen, sondern bringen auch eine Einschränkung der Lebensqualität mit sich. Laut aktuellen Leitlinien werden diese häufigen Erkrankungen lediglich symptomatisch durch eine angepasste Analgesie und eine Flüssigkeitsgabe sowie zunächst durch eine kalkulierte Antibiotikatherapie behandelt. Die Notwendigkeit einer Katheteranlage ist nicht eindeutig definiert, sondern wird durch den betreuenden Arzt mithilfe der klinischen Parameter des Patienten eingeschätzt und entschieden. In dieser urologischen klinischen Studie geht es um die Therapie von komplizierten Harnwegsinfektionen und die Optimierung sowie Standardisierung ihres derzeitigen Therapieregimes. Zunächst wurde das Studiendesign auf eine prospektive Studie ausgelegt, in der 50 männliche und 50 weibliche Patienten eingeschlossen werden sollten. Durch eine 1:1-Randomisierung sollte diesen Patienten ein Katheter (Männer: suprapubischer Katheter, Frauen: transurethraler Katheter) oder kein Katheter zugeteilt werden. Die eingeschlossenen Patienten sollten im Hinblick auf ihre klinischen Parameter (C-reaktives Protein (CRP), Leukozyten, Körpertemperatur, Krankenhausaufenthalt, Antibiotikagabe etc.) untersucht werden und die Männer in ein Follow-Up, eine Nachsorgeuntersuchung, eingeschlossen werden. Allerdings musste diese Studie aufgrund einer zu geringen Patientenrekrutierung beendet und durch eine retrospektive Studie ersetzt werden.

In der retrospektiven Untersuchung wurden dann wie geplant 50 männliche und 50 weibliche Patienten mit komplizierten Harnwegsinfektionen untersucht. Das Augenmerk wurde aufgrund der statistischen Berechnungen auf die Krankenhausaufenthaltsdauer gelegt.

Hierbei konnte für das männliche Patientenkollektiv gezeigt werden, dass die Anlage eines Katheters zu einem verlängerten Krankenhausaufenthalt von 1,36 Tagen führt. In der Subgruppe der Männer > 63 Jahre konnte kein Zusammenhang zwischen einer Katheteranlage und dem Krankenhausaufenthalt festgestellt werden, wohingegen bei der Subgruppe $\leq 63$ Jahren herausgefunden wurde, dass 
eine Katheteranlage zu einem um 2,0 Tage verlängerten Krankenhausaufenthalt führt.

Bei den weiblichen Patienten konnte kein Zusammenhang zwischen der Katheteranlage und dem Krankenhausaufenthalt gefunden werden.

Zur statistischen Auswertung wurde die Art der Katheteranlage nicht berücksichtigt. Die ursprüngliche Fragestellung, ob die Anlage eines suprapubischen Katheters einen Vorteil für den Patienten birgt, kann somit aufgrund des retrospektiven Studiendesigns nicht abschließend beantwortet werden. Die Durchführung einer erneuten prospektiven, multizentrischen Studie ist in Planung. 


\section{Abstract:}

Complicated urinary tract infections (UTI) such as prostatitis and epididymitis in men or pyelonephritis in women are a common reason for urological hospital admissions. For the patient, this means not only physical discomfort (pain) but also a restriction of their quality of life. According to guidelines, patients are treated symptomatically with adapted analgesia and a calculated antibiotic therapy. So far, it is not clearly defined whether the treatment should also include a urinary catheter and by which criteria the decision about a catheter should be made. Therefore, we designed this clinical trial to optimize and standardize the therapeutic regime. Initially, a prospective trial including 50 male and 50 female patients was planned. By 1:1-randomization, patients were to be assigned to two groups (suprapubic catheter or no catheter). The patients were afterwards to be compared according to several parameters (e.g. CRP, leucocytes, body temperature, hospitalization time, use of antibiotics etc.). However, the primary endpoint of 100 patients was not reached, which is why we instead went for a retrospective design. This also included 50 male and 50 female patients with complicated UTI. Main focus was hospitalization time.

We could find that male patients with catheter were admitted 1.36 days longer compared to patients without catheter. In the subgroup of men $>63$ years no relationship between catheterization and admission time was established whereas in the subgroup $\leq 63$ years patients with a catheter system stay 2.0 day longer in hospital than patients without a catheter.In case of female patients, this correlation could not be found. The initial question of whether a suprapubic catheter would be beneficial could not be answered in the retrospective analysis.

Therefore, a prospective, multicentric study is in planning. 


\section{Einleitung}

Komplizierte Harnwegsinfektionen (HWI) wie Prostatitis, Epididymitis und Pyelonephritis sind häufige Krankheitsbilder der Urologie, die den Alltag der Patienten enorm einschränken können. Durch ein frühes und optimales Therapieregime können die Infektion und die belastenden Symptome schnell gebessert sowie die Lebensqualität der Patienten wiederhergestellt werden.

\section{$1.1 \quad$ Fragestellung und Zielsetzung}

Für die Anwendung eines Katheters bei komplizierten Harnwegsinfektionen, insbesondere eines suprapubischen Katheters (SPK), gibt es keine eindeutig definierten medizinischen Indikationen. Die European Association of Urology (EAU) Guidelines on Urological Infections erwähnen lediglich, dass ein suprapubischer Katheter generell empfohlen ist, er aber zurückhaltend eingesetzt werden soll, um die Gefahr einer chronischen Prostatitis zu verhindern (Yoon et al. 2012).

Die Indikation zur Katheteranlage trifft der betreuende Arzt individuell für den Patienten. Bei einem Harnstau erhält der Patient auf jeden Fall eine dauerhafte Harnableitung. In allen anderen Fällen wäre es von Vorteil, wenn genauere Angaben für den therapeutischen Algorithmus einer Katheterisierung bei komplizierten Harnwegsinfektionen vorhanden wären.

Eine hierzu initial durchgeführte prospektive Studie zur Erfassung von 100 Patienten in der Urologie der Universitätsmedizin Göttingen (UMG) war aufgrund zu geringer Teilnehmerzahlen nicht auszuwerten. Die erhobenen Daten waren nach ersten Analysen vielversprechend, benötigen aber ein größeres Patientenkollektiv, um ein aussagekräftiges Ergebnis zu erzielen.

Aus diesem Grund wurden in dieser Arbeit retrospektiv erhobene Daten von 100 Patienten aus der Klinik für Urologie der Universitätsmedizin Göttingen untersucht. Hierbei wurde analysiert, ob die Anwendung eines Katheters bei komplizierten Harnwegsinfektionen von Männern, insbesondere eines suprapubischen Katheters, einen Benefit für den Patienten in Hinblick auf Krankenhausaufenthalt, Lebensqualität und Entzündungsparameter darstellt. 
Außerdem wurde begleitend bei Frauen mit Pyelonephritis, die sich in stationärer urologischer Behandlung der Universitätsmedizin Göttingen befanden, der potenzielle Nutzen einer transurethralen Katheteranlage untersucht.

\subsection{Anatomische Grundlagen}

\subsubsection{Die Prostata}

Die Prostata ist eine der vier akzessorischen Geschlechtsdrüsen des Mannes. Sie ist kaudal der Harnblase gelegen und umschließt den oberen Teil der Urethra. Die Samenleiter sowie die Ausführungsgänge der Samenblase münden in die Prostata (Lippert 2011,Schünke et al. 2012). Aufgabe der Prostata ist, ein Sekret zu produzieren, welches für die Beweglichkeit der Spermien verantwortlich ist. Außerdem entsteht in der Drüse das Prostata-spezifische Antigen (PSA), welches das Ejakulat dünnflüssiger macht. Dieser Marker wird häufig zur Diagnostik herangezogen. Das produzierte Sekret wird während der Ejakulation durch kräftige Kontraktionen der glatten Muskulatur vollständig ausgestoßen.

\subsubsection{Der Nebenhoden}

Der Nebenhoden (Epididymis) ist ein paarig angelegtes Geschlechtsorgan des Mannes, der sich dorsal des Hodens befindet und anatomisch in Kopf, Körper und Schwanz eingeteilt wird. Der Nebenhoden wird vom Nebenhodengang (Ductus epididymidis) durchzogen, der anschließend in den Samenleiter (Ductus deferens) übergeht.

Die Spermien werden im Hoden gebildet und über Samenkanäle (Ductuli efferentes testes) in den Nebenhoden geleitet. Dort findet innerhalb von zwei Wochen die Reifung der Spermien statt, die über kurze Zeit gespeichert werden, bis sie während der Ejakulation durch die Samenleiter austreten (Lippert 2011).

\subsubsection{Das Nierenbecken}

Die paarig angelegte Niere befindet sich unterhalb des Diaphragmas im Retroperitoneum. Sie ist bohnenförmig und hat eine Länge von ca. $11 \mathrm{~cm}$ sowie eine Breite von $7 \mathrm{~cm}$. 
Anatomisch gliedert sich die Niere in eine äußere Nierenrinde, anschließende Nierenpyramiden und das Nierenmark (Lippert 2011). Über die Pyramiden wird der gebildete Harn in den Nierenkelch geleitet. Von dort fließt er weiter in das Nierenbecken, das als eine Art Sammeltrichter fungiert. Hier wird der Urin aufgefangen und anschließend über den Ureter zur Blase transportiert (Schünke et al. 2012). Weitere zahlreiche Funktionen der Niere sind unter anderem die Regulation des Salz- und Elektrolythaushalts, die Regulation des Säure-Basen-Haushalts sowie die Hormonproduktion (Lippert 2011).

Bei aufsteigenden Harnwegsinfektionen, die bei Frauen aufgrund der „kürzeren“ Länge der Urethra häufiger vorkommen, ist das Nierenbecken durch aufsteigende Keime gefährdet.

\subsection{Krankheitsbilder}

Diese klinische Studie beschäftigt sich mit drei verschiedenen Erkrankungen, die als komplizierte Harnwegsinfektionen zusammengefasst werden können. Dazu gehören die akute Prostatitis und Epididymitis beim Mann sowie die akute Pyelonephritis der Frau, auf die im nachfolgenden Absatz detailliert eingegangen werden soll.

\subsubsection{Prostatitis}

Als Prostatitis bezeichnet man eine Entzündung der Prostata (Margreiter und Shariat 2016).

Die Prävalenz der Prostatitis variiert je nach Studienlage zwischen 9 bis $16 \%$ (Collins et al. 2002; Margreiter und Shariat 2016).

Klassischerweise unterteilt man das Prostatasyndrom nach den National Institutes of Health $(\mathrm{NIH})$ in vier verschiedene Kategorien (Hautmann 2010, Krieger et al. 1999):

Akute bakterielle Prostatitis

Chronische bakterielle Prostatitis

Chronisch abakterielle Prostatitis /

Chronisches Schmerzsyndrom des Beckens

$>\quad$ Asymptomatische entzündliche Prostatitis 
In dieser internationalen Klassifikation ist die chronische bakterielle Prostatitis definiert durch eine mindestens drei Monate bestehende Symptomatik. In der dritten Kategorie, der chronischen abakteriellen Prostatitis, lässt sich zusätzlich kein Erreger nachweisen. Die vierte Kategorie ist ein reiner Zufallsfund ohne entsprechende Symptomatik (Krieger et al. 1999).

Diese Studie beschäftigt sich ausschließlich mit der akuten bakteriellen Prostatitis.

Die akute Prostatitis ist eine schmerzhafte Entzündung der Prostata und stellt die häufigste urologische Erkrankung bei Männern unter 50 Jahren dar (Blacklock 1991). Die Prävalenz der Prostataentzündung weist mit $8-16 \%$ eine große Spannbreite auf (Davis und Silberman 2018).

Die Patienten klagen typischerweise über starke Schmerzen im Bereich von Skrotum, Penis oder Harnblase. Außerdem geht die Erkrankung häufig mit einer Harnwegssymptomatik (Lower Urinary Tract Symptoms=LUTS), bestehend aus Pollakisurie, Dysurie und Algurie einher. Nicht selten beschreiben die Patienten eine Ausstrahlung in den Rücken (EAU-Guidelines Prostatitis/Epididymitis 2015, Ludwig 2010b). Somit ist die Prostatitis zunächst eine klinische Diagnose.

Ursache der akuten Entzündung sind über die Urethra aufsteigende, in der Regel bakterielle Erreger.

Die häufigsten ursächlichen Erreger sind verschleppte gramnegative Enterobakterien wie z. B Escherichia Coli (E. coli) (75 - 80 \%), Pseudomonas aeruginosa (10 $15 \%$ ) und Proteus mirabilis (EAU Guidelines Prostatitis/Epididymitis 2015, Margreiter und Shariat 2016). Zusätzlich müssen sexuell übertragbare Erreger vor allem bei jüngeren Patienten in Erwägung gezogen werden. Selten kann es auch zu einer Infektion durch Mycobakterium tuberculosis kommen (Hautmann 2010). Grampositive Bakterien und atpyische Erreger finden sich häufig bei chronischer Prostatitis (Davis und Silberman 2018).

Risikofaktoren für eine Prostatitis sind ein nicht therapeutisch angegangener Harnwegsinfekt sowie Harnröhrenengen, eine Katheterdauerversorgung, transrektale Prostatabiopsien und Men who have sex with men (MSM) (Margreiter und Shariat 2016).

In 5-10\% der Fälle geht die akute Prostatitis in eine chronische Form über, welche die Lebensqualität der Patienten sehr verschlechtert (Hautmann 2010). Risikofak- 
toren für eine Chronifizierung sind ein vermehrter Alkoholabusus, Diabetes mellitus und ein großes Prostatavolumen (Davis und Silberman 2018).

Es wurde beschrieben, dass eine früh eingesetzte Antibiotikatherapie die Chronifizierung der Prostatitis verhindern kann (Davis und Silberman 2018).

Im diagnostischen Algorithmus steht zunächst, neben der ausführlichen Anamnese, die körperliche Untersuchung sowie die digital-rektale Untersuchung (DRU) aller Patienten im Vordergrund. In der DRU findet sich eine druckschmerzhafte, warme und geschwollene Prostata (Ludwig 2010b).

Anschließend folgen Laboruntersuchungen bezüglich der Entzündungsmediatoren (CRP, Leukozyten, ggf. PCT). Klassischerweise finden sich stark erhöhte Entzündungsparameter mit einer Leukozytose sowie eine erhöhte Blutsenkungsgeschwindigkeit (BSG). Ein Keimnachweis im Urin gelingt jedoch nur in $5-10 \%$ der Fälle (Weidner et al. 1991b).

Zusätzlich kann das Prostata-spezifische Antigen (PSA) im Blut bestimmt werden, welches aufgrund seiner geringen Spezifität lediglich unterstützend eingesetzt werden sollte. Ein normaler PSA-Wert schließt eine akute Prostatitis nicht aus. Ebenso wenig muss ein erhöhtes PSA für ein entzündliches Geschehen sprechen. Neben der Labordiagnostik kann die 4 - Gläser-Probe bzw. heute häufiger verwendet die 2 - Gläser Probe, bestehend aus der Analyse des Mittelstrahlurins und dem Exprimat nach Prostatamassage, hinweisgebend sein. Das Ergebnis der 2 - Gläser-Probe differenziert zwischen der Urethritis (positive erste Probe), Zystitis (positive zweite Probe) und Prostatitis (positive dritte oder vierte Probe) ( Margreiter und Shariat 2016).

Laut den EAU-Leitlinien werden die Ejakulatanalysen aufgrund der geringen Sensitivität, vor allem im Vergleich zur 2 - Gläser-Probe, nicht mehr empfohlen (EAUGuidelines Prostatitis/Epididymitis 2015).

Zur erweiterten Diagnostik kann die transrektale Ultraschalluntersuchung (TRUS) hinzugezogen werden. Hierdurch können gefährliche Komplikationen wie Prostataabszesse erkannt und therapiert werden. Nach den EAU-Leitlinien gehört dieses Verfahren jedoch nicht zur Standarddiagnostik.

Die Therapie der Prostatitis besteht aus einer kalkulierten medikamentösen Therapie. Hauptmedikamentengruppe sind die Fluorchinolone, die aufgrund ihrer gu- 
ten Gewebegängigkeit für die Prostata und ihres breiten Wirkungsspektrums klare Vorteile gegenüber anderen Antibiotika aufweisen (Aktories und Forth 2013).

Alternativ ist für die Initialbehandlung ein Breitspektrumpenicillin oder ein Drittgenerations-Cephalosporin empfohlen. Alle drei Medikamentengruppen können zu Beginn mit einem Aminoglykosid kombiniert werden. Nach einer initial erfolgten intravenösen (iv) Antibiotikatherapie sollte die Antibiotikatherapie oral für 2 - 4 Wochen fortgesetzt werden (Schaeffer et al. 2002)

Symptomatisch wird zudem häufig eine Analgesie mittels nichtsteroidaler Antirheumatika (NSAR) begonnen. Die Notwendigkeit einer suprapubischen Harnableitung, unabhängig von der Stauung der Nierenbeckenkelchsysteme, ist umstritten und soll durch diese Studie genauer beleuchtet werden.

\subsubsection{Epididymitis}

Als Epididymitis bezeichnet man die akute oder chronische Entzündung des Nebenhodens.

Inzidenz und Prävalenz sind trotz zahlreicher Studien unklar (EAU-Guidelines Prostatitis/Epididymitis 2015). Man geht von 25 - 65 Neuerkankungen pro 10000 Erwachsenen pro Jahr aus, gemessen im Vereinigten Königreich, den USA und Kanada (Çek et al. 2017).

Die akute Epididymitis ist die häufigste Erkrankung des Nebenhodens und geht in $15 \%$ der Fälle in eine chronische Form (> 3 Monate) über (EAU-Guidelines Prostatitis/Epididymitis 2015, Ludwig 2010a).

Bei den Krankheitsursachen differenziert man zwischen einer infektiösen sowie nichtinfektiösen Genese (Ludwig 2010a, Rupp und Leslie 2017):

Infektiös:

> Deszendierende HWI Erreger (z. B. Enterobakterien)

> Sexuell übertragbare Erreger (z. B. C. trachomatis, N. gonorrhoeae)

> Hämatogen (Septikämie, Tuberkulös) 
Nichtinfektiös (seltener):

$>$ Nach Verletzung des Sphinkters

$>$ Morbus Behçet

$>$ Nach Vasektomie

$>$ Amiodaron-Nebenwirkung

$>$ Idiopathisch (ca. $40 \%$ )

Das Erregerspektrum richtet sich nach dem Alter der Patienten. Bei Männern < 35 Jahre können häufiger sexuell übertragbare Erreger wie Chlamydia trachomatis oder Neisseria gonorrhoeae nachgewiesen werden. Bei Männern > 35 Jahre finden sich hauptsächlich gramnegative Enterobakterien wie E. coli, Proteus mirabilis oder Klebsiellen (Weidner et al. 1987, Hautmann 2010).

Risikofaktoren für eine Nebenhodenentzündung sind, wie bei der akuten Prostatitis, ein nicht therapeutisch angegangener Harnwegsinfekt sowie Harnröhrenengen, eine Katheterdauerversorgung und MSM (Hautmann 2010). Nach einer transrektalen Prostatabiospie tritt in 4 - $6 \%$ der Fälle eine anschließende Epididymitis auf (Wilbert 2009).

Die Patienten beschreiben plötzliche und massive Schmerzen des Skrotums, die meistens mit einer Schwellung und Überwärmung einhergehen. Außerdem berichten sie häufig über Pollakisurie und Dysurie. Die Betroffenen zeigen zudem häufig hohes Fieber und imponieren durch ein stark ausgeprägtes Krankheitsgefühl. Oft kommt es bei unbehandeltem Verlauf zu einer Mitbeteiligung des Hodens in Form einer Epididymorchitis. Der Nebenhoden kann dann häufig schlecht vom Hoden abgegrenzt werden (Ludwig 2010a).

Komplikationen einer akuten Nebenhodenentzündung können neben der Chronifizierung auch Abszesse, eine Hodenatrophie sowie Infertilität sein (EAUGuidelines Prostatitis/Epididymitis 2015).

Die Verdachtsdiagnose Epididymitis lässt sich mittels Anamnese sowie klinischer Untersuchung erhärten. Hier imponiert ein meist druckschmerzhafter, geschwollener und überwärmter Hodensack. Häufig verringern sich die Schmerzen beim Anheben des Hodens, was als positives Prehn-Zeichen bezeichnet wird und häufig mit einer Nebenhodenentzündung einhergeht. Dieses Zeichen stellt lediglich einen 
Hinweis dar und ist deshalb umstritten. Außerdem wird jeder Patient digital-rektal untersucht.

Im Labor zeigt sich meist eine unspezifische Entzündungsreaktion. Es sollte ebenfalls eine Urinanalyse erfolgen, bei der häufig eine Leukozyturie imponiert (Hautmann 2010).

Im transskrotalen Ultraschall sieht man ggf. einen aufgetriebenen und hyperperfundierten Nebenhoden (Wilbert 2009). Bei bestehendem Fieber sollte ebenfalls an abzunehmende Blutkulturen gedacht werden.

Das Therapieregime der Epididymitis orientiert sich an dem Vorgehen der Prostatitis. Im Vordergrund stehen hier ebenfalls die symptomatische und antibiotische Therapie.

In der antibiotischen Therapie werden laut den Europäischen Leitlinien die Fluorchinolone empfohlen. (Aktories und Forth 2013, EAU-Guidelines Prostatitis/Epididymitis 2015). Diese sind gleichzeitig gut gegen intrazelluläre Erreger wirksam. Hierfür eignen sich neben Ciprofloxacin vor allem Ofloxacin und Levofloxacin. Bei nachgewiesener Chlamydieninfektion kann die Therapie mit Doxycyclin für mindestens 2 Wochen fortgesetzt werden. Alternativ können Makrolide verwendet werden (EAU-Guidelines Prostatitis/Epididymitis 2015).

Hinzu kommt, dass die Patienten strikte Bettruhe halten sollen. Sie sollen den Hoden hochlagern und kühlen. Zur Schmerztherapie eignen sich NSAR.

Während die Anlage eines transurethralen Dauerkatheters aufgrund der Gefahr der Verschleppung von Keimen kontraindiziert ist, kann über die Anlage eines SPK nachgedacht werden. Die Anlage ist indiziert bei vorhandenem Restharn, zusätzlicher Prostatitis oder bei fehlendem Therapieerfolg trotz adäquater Antibiotikatherapie (Ludwig 2010a).

Bereits 1960 konnte Dietz zeigen, dass die Spermienqualität bei Nichtbehandlung einer Nebenhodenentzündung zunehmend schlechter wird (Dietz 1960). Eine Studie 1991 von Osegbe zeigte, dass sich die Spermienqualität nach Behandlung zwar verbessern kann, es aber nach 3 - 6 Monaten zu keiner weiteren Erholung kommt (Osegbe 1991).

Eine neuere Studie 2017 hat gezeigt, das $10 \%$ der Patienten mit akuter Epididymitis anschließend eine Azoospermie und sogar $30 \%$ eine Oligoazoospermie entwickeln (Schuppe et al. 2017). 
Verschiedene Studien weisen darauf hin, dass $40 \%$ der Patienten dauerhaft eine eingeschränkte Spermienkonzentration davontragen (Osegbe 1991; Weidner et al. 1991a; Ludwig und Haselberger 1977).

Deshalb ist es wichtig, ein optimales Therapieregime anzustreben.

\subsubsection{Pyelonephritis}

Die Pyelonephritis ist eine Erkrankung, die häufig aus einer unbehandelten Zystitis hervorgeht und ebenfalls zu den komplizierten Harnwegsinfekten gezählt wird. Dabei sind Frauen, aufgrund der anatomisch kürzeren Urethra und der dadurch häufiger auftretenden Zystitis, häufiger betroffen als Männer (Füssle und Sziegoleit 2001). $75 \%$ der Patienten mit Pyelonephritis weisen vorausgegangene Zystitiden auf (Vahlensieck 2010), bei denen durch eine frühzeitige Antibiotikatherapie eine Pyelonephritis verhindert werden kann (Bleidorn et al. 2010; Gágyor et al. 2015; Gágyor et al. 2012)

Die Prävalenz einer akuten Nierenbeckenentzündung betrug 2012 laut S3-Leitlinie für alle Frauen älter als 12 Jahren 0,16 \% (AWMF-Leitlinie unkomplizierte Harnwegsinfektionen 2017 ,Foxman and Frerichs 1985). Frauen im Alter zwischen 20 - 30 Jahren sind am häufigsten betroffen (Foxman and Frerichs 1985). Bei Patientinnen in der Postmenopause liegen Daten vor, die eine Ein-Jahres-Prävalenz von 1,19 / 1000 Patientinnen angeben (van der Linden et al. 2004).

Durch die enge anatomische Lagebeziehung zwischen Urethra und Anus kommt es häufig zur Verschleppung von Darmbakterien wie z. B. E. coli, Enterokokken, Proteus mirabilis, Klebsiellen sowie Pseudomonas aeruginosa. Die Infektion kann somit durch aufsteigende Keime hervorgerufen werden, seltener auch durch lymphogene oder hämatogene Verschleppung (Petry und Gillitzer 2009).

Die Patienten leiden unter typischen Harnwegssymptomen wie Pollakisurie, Dysurie und Algurie, imponieren teils mit hohem Fieber über $38^{\circ} \mathrm{C}$ und zeigen bei der körperlichen Untersuchung ein klopfschmerzhaftes Nierenlager (Hautmann 2010). Häufig ist die Entzündung von unspezifischen Symptomen wie Schüttelfrost, Übelkeit, Erbrechen und Tachykardie begleitet (Merkle 1997). Bei komplizierten Nierenbeckenentzündungen können ebenfalls unspezifische abdominelle Beschwerden, Übelkeit und Erbrechen sowie Diarrhoen auftreten (Vahlensieck 2010). 
Neben der ausführlichen Anamnese und körperlichen Untersuchung zeigt das Labor eine typische Entzündungskonstellation. Meist findet sich ein erhöhtes CRP und eine erhöhte BSG sowie eine Leukozytose oder seltener eine Leukozytopenie, ggf. kann man einen Anstieg des Kreatinins beobachten (Hautmann 2010).

Der meist bei Aufnahme und typischer Beschwerdesymptomatik abgenommene Urinstatus wird hinsichtlich der Granulozytenesterase-Aktivität (GE-Aktivität), des Proteingehalts sowie auf das Vorhandensein von Leukozyten und Nitrit untersucht. Häufig beobachtet man eine erhöhte GE-Aktivität und eine Nitrit-Positivität.

Eine vor Antibiotikabeginn angelegte Urinkultur kann eventuell den verursachenden Keim aufzeigen und sollte in jedem Fall erfolgen.

Bei fieberhaften Patienten sollte in jedem Falle eine Blutkultur abgenommen werden, um eine schwere Komplikation wie eine Urosepsis frühzeitig zu erkennen und zu behandeln.

Sonographisch kann die Entzündung mit einer Ektasie des Nierenbeckenkelchsystems, einer Schwellung des Nierenparenchyms sowie in der Dopplersonographie mit einer Hyperperfusion der Niere einhergehen (EAU-Guidelines Pyelonephritis 2015).

Aufgrund des hohen Leidensdrucks der Patienten besteht die Therapie zunächst aus Symptomlinderung und Bettruhe sowie kausal aus einer empirischen Antibiotikagabe, Antiphlogistika und Flüssigkeitsgabe.

Eine empirische Antibiotikagabe sollte frühestmöglich begonnen werden, da das Risiko für eventuelle Nierenschäden mit der Dauer einer unbehandelten Erkrankung steigt (AWMF-Leitlinie unkomplizierte Harnwegsinfektionen 2017).

Bei leichten bis mittelschweren Symptomen kann eine orale Antibiotikatherapie in Erwägung gezogen werden. Mittel der ersten Wahl sind die Fluorchinolone (Ciprofloxacin, Levofloxacin), solange die Resistenzrate von E. coli kleiner $10 \%$ ist (Talan et al. 2000). Da laut aktueller S3-Leitlinie 2017 die Resistenzrate für Ciprofloxacin bei $E$. coli bei $15,3 \%$ und bei Cefpodoxim lediglich bei $8,7 \%$ liegt, sollte Ciprofloxacin nicht mehr Mittel der ersten Wahl darstellen (AWMF-Leitlinie unkomplizierte Harnwegsinfektionen 2017). Cephalosporine der Drittgeneration wie Cefpodoxim weisen ein gutes Wirkspektrum und geringe Resistenzraten auf und stellen somit ein besseres Mittel der ersten Wahl dar. Bei Unverträglichkeit gegen die oben genannten Antibiotikagruppen können Aminopenicilline in Kombination mit 
Betalaktamaseinhibitoren verabreicht werden. Sollte nach drei Tagen keine Besserung eingetreten sein, muss auf eine intravenöse Antibiotikagabe umgestellt werden (AWMF-Leitlinie unkomplizierte Harnwegsinfektionen 2017).

Bei schwereren Symptomen muss initial eine stationäre intravenöse Antibiotikatherapie erfolgen. Hierfür werden ebenfalls noch primär, trotz hoher Resistenzentwicklung, Fluorchinolone (Ciprofloxacin, Levofloxacin) eingesetzt. Mittel der zweiten Wahl sind Cephalosporine der Drittgeneration, Aminoglykoside oder Carbapeneme. (EAU-Guidelines Pyelonephritis 2015, Vahlensieck 2010). Gegebenfalls muss nach Erhalt des Antibiogramms das Antibiotikum (AB) resistenzgerecht angepasst werden.

Bei Reflux oder Überlaufblase mit Harnstau sollte umgehend aufgrund des erhöhten Risikos eines vesikoureteralen Reflux eine transurethrale Harnableitung erfolgen, um das weitere Aufsteigen von Keimen sowie eine Urosepsis zu verhindern. Genauere Indikationen für eine Harnableitung sind in den Leitlinien nicht beschrieben. Im klinischen Alltag erhalten jedoch viele Patienten bei oben beschriebenen Symptomen eine dauerhafte Harnableitung. Über die Anlage des Katheters wird individuell für den Patienten vom behandelnden Stationsarzt entschieden. Um den potenziellen Nutzen der Katheteranlage zu überprüfen und ein optimales Therapieregime für Ärzte und Patienten zu erhalten, wurde diese Studie konzipiert.

\subsection{Harnableitung}

Viele medizinische Indikationen machen es notwendig, einen Katheter zu verwenden. Auf der einen Seite steht die diagnostische Möglichkeit der sterilen Uringewinnung (z. B. bei postmenopausalen Frauen, wo ein Mittelstrahlurin häufig keine zuverlässige Aussage erlaubt) im Vordergrund. Der häufigere Grund für eine Katheteranlage ist jedoch therapeutisch. Grundsätzlich unterscheidet man zwei Arten der Harnableitung, transurethral oder suprapubisch. Beide Verfahren werden im nachfolgenden Kapitel genauer vorgestellt. 


\subsubsection{Transurethrale Harnableitung}

Der transurethrale Dauerkatheter (DK) ist die erste Wahl zur Harnableitung und kann in vielen klinischen Situationen notwendig sein.

Vorteil sind die einfache, schnelle Anlage und die vergleichsweise wenigen Kontraindikationen wie urologische Infektionen der unteren Harnwege (Prostatitis und Epididymitis), Harnröhrenengen sowie Harnröhrenverletzungen. Hierbei muss auf einen suprapubischen Katheter zurückgegriffen werden (Sökeland et al. 2000). Außerdem stehen viele verschiedene Kathetergrößen zur Verfügung, sodass eine optimale Anpassung möglich ist (Sökeland et al. 2000).

Nachteil ist, dass ungenügende Hygienebedingungen bei einem lange liegenden Katheter häufiger zu Infektionen führen. Durch die aufsteigenden Keime besteht ein erhöhtes Risiko für eine nachfolgende Prostatitis, Epididymitis oder Pyelonephritis. Im schlimmsten Fall kann durch die Verschleppung von Keimen eine lebensgefährliche Urosepsis entstehen. Dabei haben Frauen ein 2,5 - fach höheres Infektionsrisiko (Sökeland et al. 2000).

Außerdem empfinden manche Patienten einen transurethralen Katheter als störend und einschränkend.

Die Komplikationen bei der Anlage eines transurethralen Katheters reichen von Verletzungen der Harnröhre bis hin zu Verstopfung des Katheters, Harnröhrenstrikturen, aufsteigenden Infektionen oder Viae Falsae (Sökeland et al. 2000).

\subsubsection{Indikationen}

Wie bereits in Kapitel 1.4 erwähnt, gibt es grundsätzlich zwei verschiedene Indikationen für einen transurethralen Dauerkatheter, die diagnostische und therapeutische Indikation. Therapeutisch wird der DK in allen Fachbereichen angewandt.

Die häufigsten Indikationen sind die Anlage bei Harnverhalt, eine prä- bzw. postoperative Anlage oder die Flüssigkeitsbilanzierung vor allem bei älteren Patienten. Auch zur Blasenspülung ist der DK Mittel der ersten Wahl. Außerdem kann der transurethraler Dauerkatheter eventuell bei palliativen Patienten die Lebensqualität verbessern (AWMF-Leitlinie Harndrainage 2015).

\subsubsection{Kontraindikationen}

Obwohl der DK ein gutes Instrument zur dauerhaften Harnableitung darstellt, gibt es einige Kontraindikationen, die eine alternative Harnableitung nötig machen. 
Kontraindikationen für diese Harnableitung sind:

> Urologische Infektionen der ableitenden Harnwege (Prostatitis, Epididymitis)

$>$ Harnröhrenengen

$>$ Harnröhrenverletzungen

$>$ Harnröhrenabriss

\subsubsection{Suprapubische Harnableitung}

Ein suprapubischer Blasenkatheter stellt eine Möglichkeit dar, den Urin des Patienten mittels Punktion dauerhaft über die Bauchdecke aus der Harnblase abzuleiten. Somit ist es möglich die physiologische Harnableitung über die Harnröhre zu umgehen.

Im Gegensatz zum DK besteht der große Vorteil in der einfacheren Hygiene, der besseren Mobilität des Patienten und den selteneren schwerwiegenden Komplikationen (Horgan et al. 1992; Ichsan and Hunt 1987).

Durch die dauerhafte Umgehung der Harnröhre können keine Verletzungen der Harnröhre oder Harnröhrenstrikturen entstehen.

Außerdem wird durch die Anlage eines SPK die Restharnbildung mit Keimreservoir verhindert, sodass das Infektionsrisiko für Prostata und Nebenhoden sinkt. Zusätzlich empfinden Patienten den SPK als geringere Einschränkung im Alltag (Sökeland et al. 2000).

Dennoch gibt es auch einige Nachteile beim SPK zu beachten. Der SPK weist einige Kontraindikationen auf, die eine Anlage verhindern (Kapitel 1.4.2.3). Der SPK muss von einem Arzt unter sterilen Bedingungen gelegt werden und kann nicht an das Pflegepersonal delegiert werden. Außerdem treten zwar weniger Komplikationen auf, diese sind allerdings wesentlich schwerwiegender als beim transurethralen Katheter (Sökeland et al. 2000).

Komplikationen beim SPK können perivesikale oder intravesikale Blutungen, Fehlpunktionen, Verletzungen von Darm oder Gefäßen, Nachblutungen und Infektionen sein (Sökeland et al. 2000). 


\subsubsection{Anlage eines SPK}

Die Anlage eines SPK stellt einen operativen Eingriff dar, der die vorherige Aufklärung des Patienten nötig macht.

Der Patient befindet sich in Rückenlage, wobei der Kopf etwas tiefer liegen sollte. Um Komplikationen durch die Punktion zu vermeiden, ist es wichtig, dass die Harnblase mit mindestens $300 \mathrm{ml}$ Flüssigkeit gefüllt ist (Albrecht et al. 2004). Zunächst palpiert der durchführende Arzt die Harnblase oberhalb der Symphyse. Heutzutage wird zusätzlich standardmäßig der Ultraschall zur genauen Identifizierung der Punktionsstelle zur Hilfe genommen. Anschließend erfolgt eine gründliche Desinfektion der zu punktierenden Stelle und die Punktionsstelle wird mit einem sterilen Tuch abgedeckt.

Nach ausreichender Desinfektion führt der Arzt eine Lokalanästhesie durch. Anschließend erfolgt eine Punktion zwei Querfinger oberhalb der Symphyse senkrecht nach unten perkutan durch die Bauchdecke (Bonkat et al. 2011). Hierüber wird in Seldinger-Technik ein Katheter eingelegt. Dieser wird anschließend fixiert und mit einem sterilen Verband befestigt (Secchi und Ziegenfuß 2009).

\subsubsection{Indikationen}

Hauptindikationen für einen SPK sind beispielsweise die Harnableitung nach einem Eingriff an den ableitenden Harnwegen, bei frustranem Versuch einer transurethralen Katheteranlage oder bei einer Beckenfraktur, bei der man von einer potenziellen Verletzung der Urethra ausgeht (Bonkat et al. 2011). Bei einer voraussichtlichen Katheterliegedauer von mehr als fünf Tagen sollte der SPK dem DK vorgezogen werden (AWMF-Leitlinie Harndrainage 2015). Grund dafür ist eine bei Anlage eines transurethralen Katheters sich ausbildende mukoprurulente Membran zwischen dem Katheter und der Urethralschleimhaut. Diese Membran fungiert als Nährboden für Bakterien, die eine aufsteigende Infektion auslösen können. Durch die Anlage eines SPK bei voraussichtlich längerer Katheterliegedauer kann somit eine Begünstigung der Keimaszendierung vermieden werden (Sökeland et al. 2000; Bubeck 1994).

Ebenfalls weisen die EAU-Leitlinien darauf hin, dass bei Infektionen der Prostata und des Nebenhoden, die mit einer Harnretention einhergehen, neben einer transurethralen Harnableitung ebenfalls eine suprapubische Harnableitung möglich ist (EAU-Guidelines Prostatitis/Epididymitis 2015 ). 


\subsubsection{Kontraindikationen}

Kontraindikationen für einen SPK sind Tumoren im Urogenitaltrakt oder Unterbauch, Blutgerinnungsstörungen oder die Einnahme von blutverdünnenden Medikamenten, die eine Punktion aufgrund des erhöhten Blutungsrisikos unmöglich machen (Hengesbach et al. 2013).

Hauterkrankungen im Punktionsbereich sowie eine verlagerte oder kleinkapazitäre Harnblase stellen ebenfalls Kontraindikationen dar.

Bei Patienten mit starker Vernarbung durch vorrangegangene Operationen sollte ebenfalls auf die Anlage verzichtet werden.

Außerdem stellt eine Schwangerschaft eine Kontraindikation für einen SPK dar (Sökeland et al. 2000).

\subsection{Was ist Lebensqualität?}

Die Weltgesundheitsorganisation (WHO) beschreibt Lebensqualität (Quality of Life, Qol) als die subjektive Wahrnehmung eines Einzelnen, im Hinblick auf die Zufriedenheit mit der Stellung im Leben, in Bezug auf individuelle Ziele, Anliegen und Erwartungen (Harper and Power 1998).

Die Lebensqualität muss als multidimensionales Konzept verstanden werden und umfasst alle wichtigen Lebensbereiche wie körperliche, psychische, funktionale, soziale und mentale Aspekte (Bestmann 2015).

Häufig kommt es zu einer Diskrepanz zwischen der individuell empfundenen und der von anderen vermuteten Lebensqualität.

Die Lebensqualität unterliegt ständigen Veränderungen z. B. entsprechend dem Lebensalter. 


\subsection{Verfahren zur Messung der Lebensqualität}

Immer wieder stellt sich in klinischen Situationen die Frage, wie objektiv die Lebensqualität des Patienten gemessen werden kann. Grundsätzlich wird zwischen Selbst- und Fremdeinschätzung unterschieden. Die Erfassung dieser sensiblen Daten kann durch verschiedene Vorgehensweisen erfolgen. Es ist z. B. möglich, ein Interview mit dem Patienten zu führen, wobei sich die Fragen dann in die Anamnese einbauen lassen. Viel effektiver ist es, den Patienten selber mithilfe eines standardisierten, validierten oder krankheitsspezifischen Fragebogens sein Befinden erfassen zu lassen (Bestmann 2015).

Standardisierte, validierte Fragebögen bieten den Vorteil, dass sie einfach anzuwenden und beliebig oft zu wiederholen sind, um eine Entwicklung festzustellen. Außerdem entfällt der unbeabsichtigte Einfluss des Interviewers bei einem Fragebogen. Die krankheitsspezifischen Fragebögen eignen sich besonders für die Vergleichbarkeit einer Erkrankung in einem bestimmten Kollektiv, die Vergleichbarkeit zwischen verschiedenen Erkrankungen ist nicht gewährleistet. Krankheitsspezifische Fragebögen werden z. B. häufig bei Tumorpatienten benutzt (Bestmann 2015).

Häufig verwendet sind generische Fragebögen, die unabhängig von einer Erkrankung international anwendbar sind. Hierzu zählen beispielsweise das Sickness Impact Profil, der Spitzer Index oder der SF-36-Fragebogen sowie seine Kurzform als SF-12-Fragebogen (Bestmann 2015; Tarlov et al. 1989; Bergner et al. 1981; Spitzer et al. 1981).

Außerdem kann man mithilfe von Rating Scales den Gesundheitszustand erfassen. Bei den Rating Scales hat sich die Schmerzskala etabliert, bei der der Patient auf einer genau definierten Skala angeben soll, wie hoch seine Schmerzen sind. Die Schmerzintensität kann somit durch eine numerische (Zahlen von 0 - 10), eine visuelle Skala oder eine visuelle Smiley-Analog-Skala, die meist bei Kindern verwendet wird, erfragt werden.

In Kapitel 3 Material und Methoden wird genauer auf den für die prospektive Studie ausgewählten SF-12-Fragebogen sowie die Visuelle Analogskala (VAS) eingegangen. 


\section{Versuchsplanung}

\subsection{Studiendesign}

In dieser prospektiv sowie retrospektiv angelegten klinischen Studie soll auf Grundlage der Daten von 100 Patienten untersucht werden, ob eine Katheteranlage (transurethral oder suprapubisch) einen Vorteil in der Therapie komplizierter Harnwegsinfekte bringt.

\subsubsection{Prospektive Studie}

Die klinisch randomisierte prospektive Studie war auf ein Jahr ausgelegt. Die Patientendaten wurden pseudonymisiert erfasst. Insgesamt sollten in der Studie 100 Patienten erfasst werden, die jeweils in zwei Arme (Harnableitung vs. keine Harnableitung) randomisiert wurden.

Eine Übersicht über das Studiendesign findet sich in Abbildung 1.
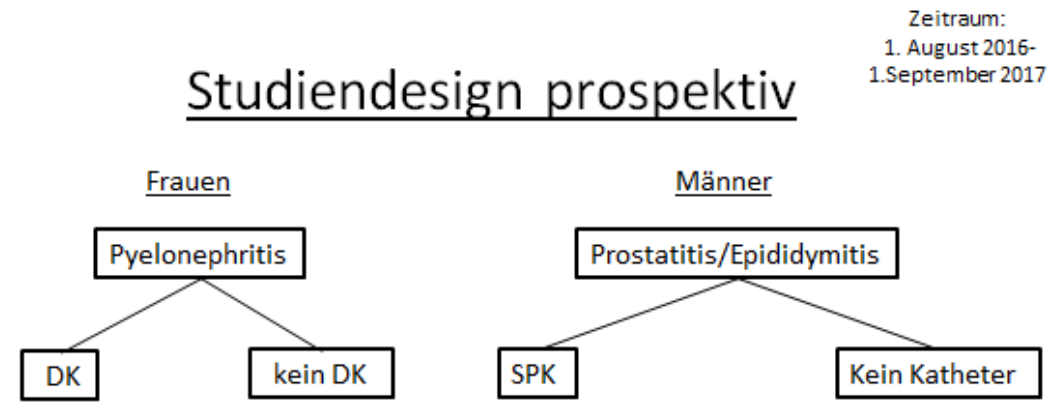

\section{Abbildung 1 Studiendesign prospektiv}

\subsubsection{Retrospektive Studie}

Da sich im Verlauf der prospektiven Studie abzeichnete, dass sich nicht ausreichend Patienten mit Prostatitis, Epididymitis und Pyelonephritis in stationärer urologischer Behandlung befanden, um das geplante Studienziel mit 100 Patienten zu erreichen, wurde entschieden die Studie durch Erfassung von retrospektiven Patientendaten zu erweitern. Eine Übersicht über das retrospektive Studiendesign findet sich in Abbildung 2. 


\section{Studiendesign retrospektiv}

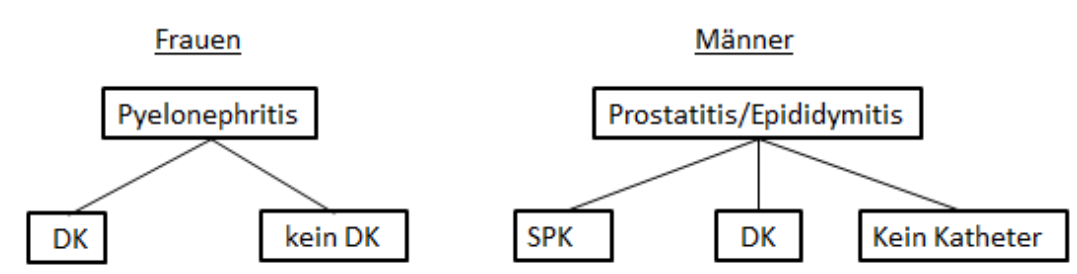

\section{Abbildung 2 Studiendesign retrospektiv}

\subsection{Studienvorbereitungen / Fallzahlabschätzung}

Vor Beginn der Studie wurde das Einverständnis der Ethikkommission Göttingen schriftlich eingeholt. Die Erlaubnis für die prospektive Studie liegt seit dem 16.06.2016 vor (Ethikvotum Nr. 25/4/16). Der zugehörige Ethikantrag befindet sich im Anhang.

Im Vorfeld wurde mit Hilfe des Göttinger Instituts für Medizinische Statistik eine Analyse zur Fallzahlabschätzung der prospektiven Studie durchgeführt. Für die Zuteilung des Patientenkollektivs wurde eine 1:1-Randomisierung benutzt.

Die Analysen wurden auf Grundlage eines erfassten Probekollektives der Universitätsmedizin (UMG, 2. Halbjahr 2015) durchgeführt.

Mittels eines t-Tests wurde errechnet, wie viele Patienten in die Studie eingeschlossen werden müssen, um ein aussagekräftiges Ergebnis mit einem Signifikanzniveau von $\alpha=5 \%$ zu erhalten.

Der t-Test wurde mit G-Power (Version 3.1.9.2, Franz Faul, Universität Kiel, Deutschland) mit einer Effektgröße von 0,57 und einer Power von 80 \% durchgeführt.

Durch die eingereichten Patientendaten ergab sich, dass 19 Patienten erfasst werden müssen, um mittels einer Leukozytendifferenz von 4,07 Tsd./mikroliter (Tsd./mikrol), mit einer Standardabweichung (SD) von 5,9 Tsd./mikrol, ein signifikantes Ergebnis zu erzielen.

Aufgrund dieser Annahme wurde entschieden, dass die bei Planung der Studie überlegte Patientenanzahl von 50 männlichen und 50 weiblichen Patienten (die auf der Hochrechnung der behandelten Patienten des letzten halben Jahres 2015 beruhte) ein ausreichend großes Patientenkollektiv darstellt. 


\section{Material und Methoden}

\subsection{Prospektive Studie}

Da aufgrund einer zu geringen Patientenrekrutierung ausschließlich die retrospektive Studie ausgewertet und analysiert wurde, wird im folgenden Abschnitt nur kurz auf die ursprünglich geplanten Rahmenbedingungen der prospektiven Studie eingegangen.

\subsubsection{Ausschlusskriterien}

Aus der Studie ausgeschlossen wurden Patienten mit jeglicher Kontraindikation für einen Katheter. Hierzu gehören:

\section{Studie der Männer:}

Tumore im Urogenitaltrakt oder Unterbauch

Blutgerinnungsstörungen

Einnahme blutverdünnender Medikamente

Hauterkrankungen im Punktionsbereich

Kleinkapazitäre Harnblase

Starke Vernarbungen

Restharnmenge $>300 \mathrm{ml}$

Harnröhrenengen

Harnröhrenverletzungen

Harnröhrenabriss

Studie der Frauen:

$>\quad$ Harnröhrenengen

> Harnröhrenverletzungen

$>\quad$ Harnröhrenabriss

> Schwangerschaft

$>\quad$ Restharnmenge 


\subsubsection{Studienbedingte Dokumente}

Für die prospektive Studie wurden die Patienten zunächst mittels einer standardisierten Patientenaufklärung über Ziel und Ablauf der Studie informiert (siehe Anhang).

Außerdem wurde separat eine Einverständniserklärung für den Patienten erstellt, die der Patient zu unterzeichnen hatte. Die Einverständniserklärungen waren in einem sicheren Raum aufbewahrt und nur für Mitarbeiter der Studie zugänglich. Die Einverständniserklärung befindet sich ebenfalls im Anhang.

Zur besseren Übersicht für den Patienten wurde ein kurzes Fließschema erstellt. Außerdem stand ein ausführliches Fließschema für ärztliches Personal zur Verfügung, in dem alle Schritte der Studie genauestens aufgelistet waren. Die Ablaufschemata für Ärzte befinden sich in Abbildung 5 und 6 . Die Ablaufschemata für die Patienten befinden sich im Anhang.

Studienpatienten sollten zur Dokumentation nach Einschluss in die Studie und nach der Entlassung den SF-12-Fragebogen sowie eine Visuelle Analogskala ausfüllen.

\subsubsection{Der SF-12-Fragebogen}

Dieser Fragebogen ist als Kurzform des SF-36-Fragebogens ein Messinstrument, welches der Erfassung der subjektiven Lebensqualität der Patienten dient (SF-36Fragebogen 2017). Während der SF-36-Fragebogen 36 Fragen zur Lebensqualität beinhaltet, werden diese in der Kurzform auf 12 Fragen zusammengefasst, ohne dass sich die Aussagekraft wesentlich verändert (Ware et al. 1996).

Der SF-12-Fragebogen umfasst die Selbsteinschätzung der körperlichen Funktion, der Schmerzen, der Vitalität, die soziale und emotionale Rolle, das psychische Wohlbefinden und die allgemeine Gesundheitswahrnehmung (SF-12-Fragebogen 2017). Dabei variieren die Antwortmöglichkeiten zwischen ja und nein sowie einer Einschätzung der Aussagen von z. B. trifft nie, meistens oder immer zu.

Der Fragebogen kann bei allen Patienten ab 14 Jahre angewendet werden (Berth und Balck 2003). 
Von Vorteil ist die einfache und schnelle Anwendbarkeit für den Patienten, die lediglich im Durchschnitt 2,5 Minuten brauchen, um den Fragebogen zu beantworten (Cella et al. 2010).

Aufgrund dieser Vorteile und der dabei trotzdem wesentlichen Aussagekraft wurde entschieden, dieses Messinstrument in die prospektive Studie mit einzubeziehen. Der verwendete standardisierte SF-12-Fragebogen befindet sich im Anhang.

\subsubsection{Visuelle Analogskala}

Visuelle Analogskalen sind ein häufig verwendetes Messinstrument zur Ermittlung von Schmerzen. Wie bereits oben erwähnt, werden unterschiedliche Arten von visuellen Analogskalen verwendet. Die klassische Visuelle Analogskala bezieht sich auf eine meist horizontale Linie, auf der der Patient seine Schmerzen einschätzen soll [Abbildung 3]. Diese Schmerzskala lässt sich auch mit einer SmileyAnalog-Skala verbinden [Abbildung 4].

\section{Abbildung 3 Beispiel einer Visuellen Analogskala}

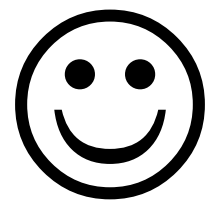

1

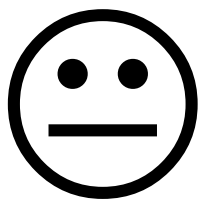

3

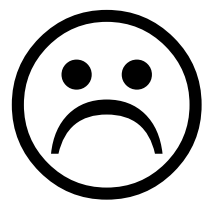

6

Kein Schmerz

Schlimmster vorstellbarer Schmerz

\section{Abbildung 4 Smiley-Analog-Skala}

\subsubsection{Studiendurchführung Männer mit Prostatitis/Epididymitis}

Nach der Diagnosestellung durch den aufnehmenden Arzt wurden die Patienten über die Studie aufgeklärt und auf eine urologische Station der UMG aufgenommen. Bei Einwilligung in die Studie wurden die Patienten anschließend mittels ei- 
ner standardisierten Randomisierungsliste einer der beiden Gruppen (SPK vs. kein Katheter) zugeordnet.

Bei Einschluss der Patienten in die Studie erhielten die männlichen Patienten einen Harnröhrenabstrich, bevor gegebenfalls der suprapubische Katheter gelegt wurde. Anschließend wurde in der Kathetergruppe, nach Aufklärung des Patienten über die Anlage eines SPK, dieser unter sterilen Bedingungen gelegt.

Außerdem wurde am dritten Tag nach Aufnahme eine zusätzliche Urinprobe analysiert. Diese konnte bei den Patienten mit suprapubischem Katheter direkt über den Katheter abgenommen werden.

Die Antibiotikagabe erfolgte standardisiert für 14 Tage nach Leitlinie (Leitlinie Urological Infections 2015). Therapiert wurden alle männlichen Patienten mit dem Fluorchinolon Ciprofloxacin 500 mg 1-0-1. Patienten unter 45 Jahren erhielten zusätzlich Doxycyclin 400 mg 1-0-1.

Während des Krankenhausaufenthaltes wurden die Patienten einmal zu Beginn und zur Entlassung zu ihren Schmerzen mithilfe der Visuellen Analogskala befragt und füllten dazu einen SF-12-Fragebogen aus (siehe Anhang).

Zur Auswertung der Studie sollten neben dem Labor (Leukozyten und CRP) die Ergebnisse des SF-12-Fragebogens, die Visuelle Analogskala, die Länge des Krankenhausaufenthaltes, die verwendeten Antibiotika sowie die nachgewiesenen Erreger erfasst werden.

Als Entlasskriterien galten für die Patienten eine 48 Stunden Fieberfreiheit sowie CRP-Werte $<100 \mathrm{mg} / \mathrm{dl}$.

\subsubsection{Follow-Up}

Die männlichen Probanden erhielten bei Entlassung einen ambulanten Wiedervorstellungstermin. Bei ihnen sollte fünf Tage nach Absetzen der Antibiotika eine Follow-Up-Untersuchung erfolgen. Hier sollte gegebenfalls die suprapubische Harnableitung entfernt, eine Uroflowmetrie sowie ein Ultraschall zur Restharnbestimmung durchgeführt werden.

Für eine bessere Vergleichbarkeit war es notwendig, dass die Patienten am vierten Tag den SPK abstöpselten und in einem mitgegebenen Miktionsprotokoll der Universitätsklinik Göttingen die Restharnmengen dokumentierten, da sich so der 
Restharn genauer bestimmen lässt (siehe Anhang). Außerdem sollten die Patienten beim Follow-Up ein letztes Mal den SF-12-Fragebogen ausfüllen.

Abbildung 5 stellt den geplanten Ablauf zur Durchführung der Studie inklusive der abzunehmenden Parameter detailliert dar.

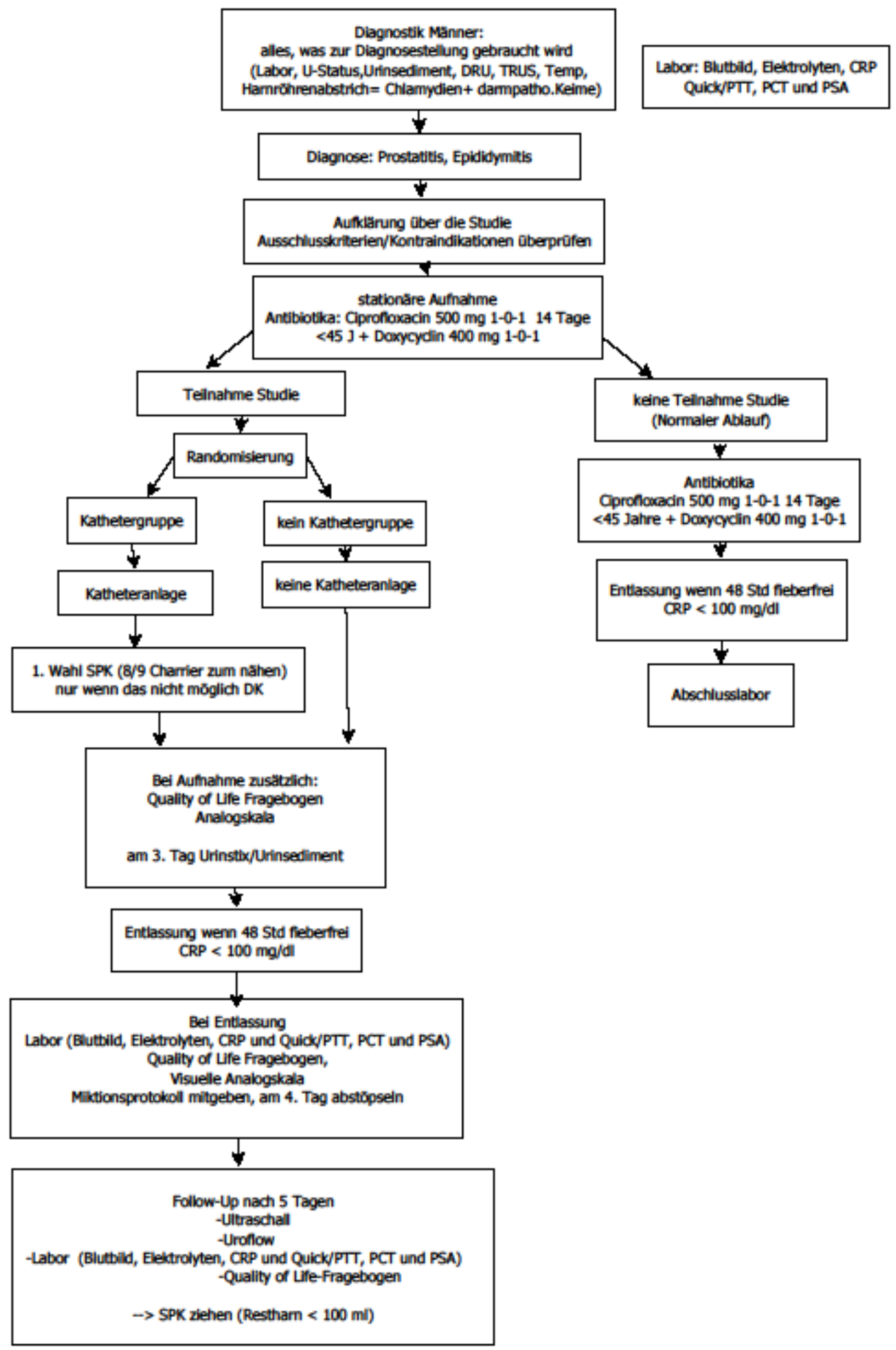




\subsubsection{Studiendurchführung Frauen mit Pyelonephritis}

Frauen, die aufgrund des oben genannten Krankheitsbildes stationär in der Urologie der UMG aufgenommen wurden, wurden ebenfalls über die Möglichkeit der Teilnahme an der Studie aufgeklärt. Bei Zustimmung und unterschriebener Einverständniserklärung wurden die Patientinnen mittels standardisierter Randomisierungsliste einer der beiden Gruppen (transurethraler vs. kein Katheter) zugeordnet. Anschließend erhielten sie gegebenfalls den transurethralen Katheter.

Die Patientinnen wurden gebeten, bei Aufnahme sowie bei Entlassung den SF-12Fragebogen sowie die Schmerzskala auszufüllen. Außerdem wurde ebenfalls am dritten Tag eine Urinprobe analysiert. Die weiblichen Patienten bekamen keine Follow-Up-Untersuchung, da bereits bei Entlassung der Katheter gezogen wurde und eine Abschlussuntersuchung erfolgte.

Frauen mit Pyelonephritis wurden für 14 Tage entweder oral mit Cefpodoxim $200 \mathrm{mg}$ oder alternativ mit einer intravenösen Cephalosporintherapie (Ceftriaxon oder Cefotaxim) 1-0-1 behandelt.

Die Auswertung der Studiendaten sollte analog den Studiendaten der männlichen Probanden ausgewertet werden, war aber aufgrund zu geringer Datensätze nicht aussagekräftig möglich.

Als Entlasskriterien galten für die Patientinnen ebenfalls eine 48 Stunden Fieberfreiheit sowie CRP-Werte $<100 \mathrm{mg} / \mathrm{dl}$.

In Abbildung 6 ist der Studienablauf der Frauen grafisch dargestellt. 


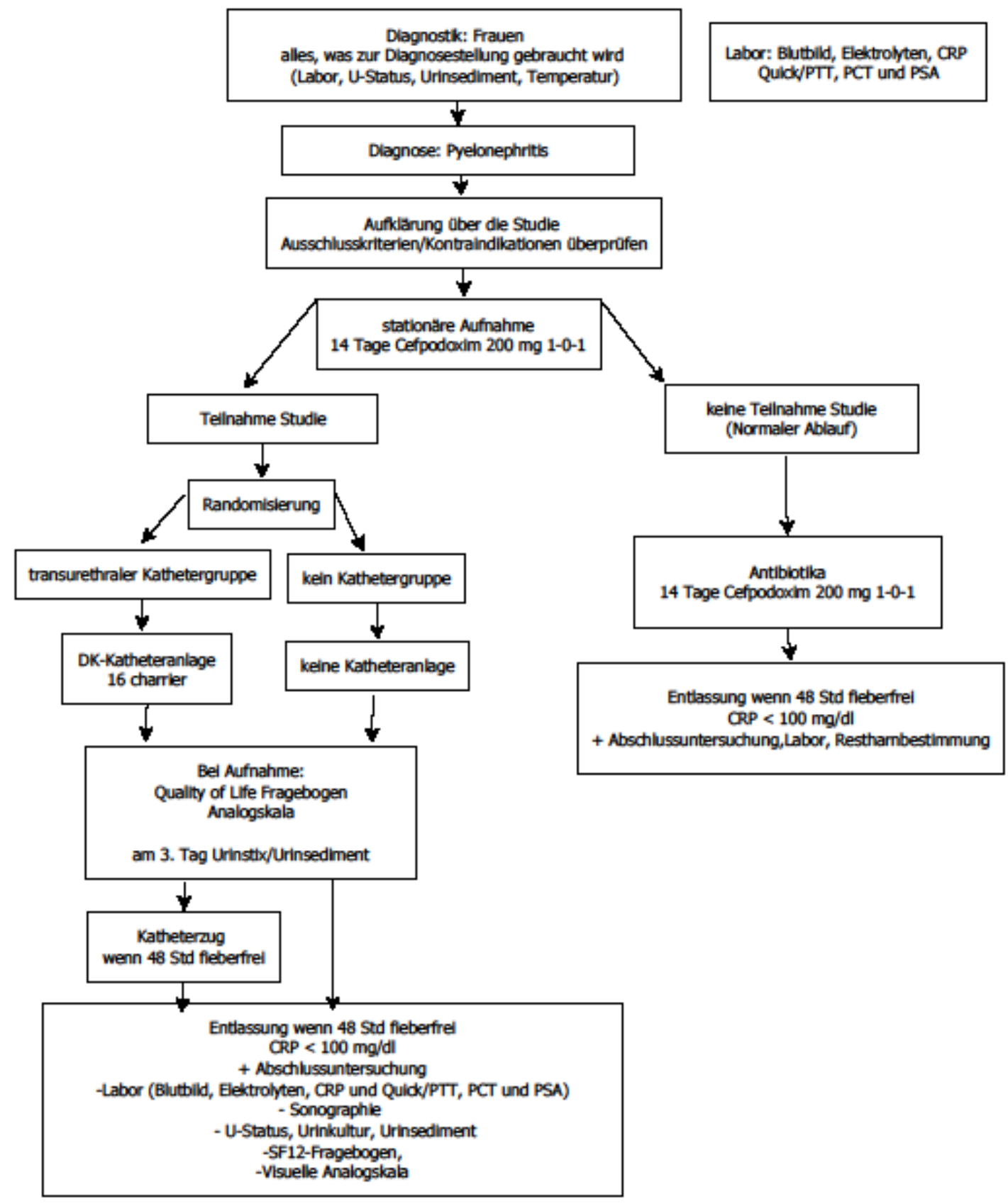

\section{Abbildung 6 Ablaufschema prospektive Studie der Frauen}




\subsubsection{Datenerfassung prospektive Studie}

Für die prospektive Studie wurden folgende Parameter der Patienten erhoben:

> Alter der Patienten

$>$ Art der Erkrankung

> Katheteranlage (ja/nein, Dauer der Katheteranlage)

> Klinische Parameter bei Aufnahme (CRP, Leukozyten, PCT, Temperatur)

> Klinische Parameter bei Entlassung (CRP, Leukozyten, PCT, Temperatur)

$>$ Ergebnisse Harnröhrenabstrich

$>$ Urinstix

> Urinstatus (Erreger sowie Resistenzen), ggf. Urinsediment

$>$ Urinuntersuchung am dritten Krankenhaustag

$>$ Krankenhausauaufenthaltsdauer

$>$ Antibiotikagabe

$>$ Ergebnisse SF-12-Fragebogen

$>$ Ergebnisse Visuelle Analogskala

$>$ Follow-Up-Untersuchung

\subsection{Retrospektive Studie}

\subsubsection{Studienvoraussetzungen}

Aufgrund der Ergänzung der Studie durch retrospektive Daten, wurde der Ethikantrag dementsprechend angepasst und eingereicht (siehe Anhang).

Die Zustimmung der Ethikkommission liegt seit dem 05.05.2017 vor (Antragsnummer 25/4/16).

Die in die Studie eingeschlossenen Patienten befanden sich alle mit einem komplizierten Harnwegsinfekt in stationärer urologischer Behandlung der Universitätsmedizin Göttingen. Die Erfassung der Daten bezieht sich auf die Jahre 2013 bis 2017 . 


\subsubsection{Ausschlusskriterien}

Aus der retrospektiven Studie wurden alle Patienten ausgeschlossen, die nicht mit den anderen Patienten vergleichbar waren. Dazu gehörten:

$>$ Patienten mit Katheterdauerversorgung

> Patienten mit intermittierendem Selbstkatheterismus

$>$ Multimorbide Patienten (> 1 urologische Vorerkrankung)

$>$ Patienten mit aktiver Tumorerkrankung

> Patienten mit Zustand nach Chemotherapie

> Patienten mit schweren Systemerkrankungen

\subsubsection{Datenerfassung retrospektive Studie}

Zur Datenerfassung wurden Frauen mit Pyelonephritis bis zum Jahr 2013 zurückverfolgt. Bei den Männern mit Prostatitis oder Epididymitis wurden die Daten bis 2015 sowie ein Datensatz aus 2014 erhoben. Somit ergibt sich ein Patientenkollektiv von 50 Männern und 50 Frauen mit komplizierten Harnwegsinfektionen.

Für die Studie wurden folgende Parameter aus den Krankenakten der eingeschlossenen Patienten erhoben:

$>$ Alter der Patienten

> Katheteranlage (ja/nein, Dauer der Katheteranlage)

> Klinische Parameter bei Aufnahme (CRP, Leukozyten, PCT, Temperatur)

> Klinische Parameter bei Entlassung (CRP, Leukozyten, PCT, Temperatur)

$>$ Urinstix

> Urinstatus (Erreger sowie Resistenzen), ggf. Urinsediment

> Krankenhausauaufenthaltsdauer

$>$ Antibiotikagabe

> Komorbiditäten (Diabetes, Arterielle Hypertonie, Tumorerkrankung)

$>$ Charlson Comorbidity Index 


\subsection{Statistische Auswertung}

In diesem Kapitel wird zunächst auf die allgemeinen Grundlagen und Annahmen zu dieser Studie eingegangen. Anschließend wird über den Umgang mit fehlenden Daten berichten und detailliert auf die Einzelheiten der statistischen Auswertung eingegangen. Die statistische Auswertung wurde unter der Anleitung von Dr. med. Annemarie Uhlig, MPH, Klinik für Urologie der UMG, durchgeführt.

\subsubsection{Allgemeine Grundlagen}

Die für die Studie relevante Expositionsvariable war die Anlage eines Katheters bzw. der Verzicht auf eine Katheteranlage. Um eine adäquate Power in den statistischen Test zu erreichen, wurde für die statistische Auswertung die Art des Katheters (SPK vs. DK) nicht berücksichtigt

Aufgrund zahlreicher fehlender Angaben zur Leukozytenzahl bei Aufnahme und Entlassung in der retrospektiven Kohorte wurde die Leukozytendifferenz als primärer Endpunkt der Studie verworfen.

Somit musste auch die zugehörige Nicht-Unterlegenheitsstudie und die zugehörige Fallzahlberechnung verworfen werden. Als neuer primärer Endpunkt wurde die Krankenhausaufenthaltsdauer in Tagen gewählt.

Als Nullhypothese $\left(\mathrm{H}_{0}\right)$ für die Studie wurde angenommen, dass die Anlage eines Katheters im Vergleich zu einem Verzicht auf die Katheteranlage zu keinem Unterschied in der Krankenhausaufenthaltsdauer in Tagen führt.

\subsubsection{Umgang mit fehlenden Daten}

Um in den statistischen Analysen eine adäquate Power zu gewährleisten, wurden für einzelne fehlende Daten möglichst konservative Annahmen gemacht:

Bei der Gruppe der Frauen wurde bei zwei Patientinnen mit unbekannter Katheterliegedauer die Aufenthaltsdauer in Tagen als Katheterliegedauer angenommen. Dieser Wert wurde gewählt, um eine möglichst konservative Schätzung zu gewährleisten. Für zwei Patientinnen waren kein CRP-Wert sowie kein LeukozytenWert bei Aufnahme verfügbar; es wurde der nächste verfügbare (Serum-) CRPund (Serum-) Leukozyten-Wert angenommen. Bei einer Patientin wurde für die 
fehlende Körpertemperatur bei Aufnahme der nächste verfügbare Wert (in diesem Fall der Entlasswert) angenommen.

Bei der Gruppe der Männer wurde bei einem Patienten mit unbekannter Katheterliegedauer ebenfalls die Dauer des Krankenhausaufenthalts als Katheterliegedauer angenommen. Für einen Patienten wurde der fehlende CRP-Wert sowie Leukozyten-Wert bei Aufnahme durch den nächsten verfügbaren Wert (einen Tag vor Entlassung) ersetzt. Bei zwei männlichen Patienten waren keinerlei CRP-Werte verfügbar. Bei vier weiteren Patienten waren keine Angaben zur Körpertemperatur verfügbar. Diese Patienten $(n=6)$ wurden aus den entsprechenden Analysen ausgeschlossen.

\subsubsection{Statistische Tests}

Die Datenerhebung wurde mittels Microsoft Excel (Excel 2010; Version 14.0.7190.5000), durchgeführt.

Sämtliche statistischen Analysen wurden mittels R (Version 3.3.1, R Core Development Team, Wien, Österreich) und RStudio (Version 1.0.153, RStudio Inc., Boston, MA, USA) durchgeführt.

Für deskriptive Statistiken wurden kontinuierliche Variablen als Mittelwert mit Standardabweichung sowie kategoriale Variablen als absolute Anzahl und Prozentwerte angegeben.

Das Signifikanzniveau wurde mit alpha $(\alpha)<0,05$ definiert. Alle $p$-Werte beziehen sich auf zweiseitige Tests.

\subsubsection{Shapiro-Wilk-Test}

Der Shapiro-Wilk-Test dient der Überprüfung der Normalverteilung der Gesamtheit einer Stichprobe (Duller 2008). Er wird durchgeführt, wenn es für nachfolgende Tests erforderlich ist, dass eine Normalverteilung vorliegt. Hierzu gehören zum Beispiel der t-Test oder die lineare Regression. Deshalb kann er auch als Vortest betrachtet werden.

Aufgrund seiner hohen Sensitivität wird dieser Test häufig für klinische Studien unabhängig von der Stichprobengröße herangezogen (Duller 2008). Aufgrund der 
Komplexität der Formeln eignet sich dieser Teil vor allem für kleine Stichprobengrößen (Holling 2010; Wetherill 1986).

$\mathrm{HO}$ als Nullhypothese nimmt an, dass eine Normalverteilung vorliegt. $\mathrm{H} 1$ stell die Alternativhypothese dar.

HO: eine Normalverteilung der Kohorte liegt vor

\section{H1: (Alternativhypothese) es liegt keine Normalverteilung vor}

Zur statistischen Berechnung wird nachfolgende Formel verwendet, wobei sich die Teststatistik W aus dem Quotienten der geschätzten Varianz b und der tatsächlichen Varianz (n-1)s ${ }^{2}$ der Stichprobe ergibt (Shapiro und Wilk 1965).

Formel 1 Berechnung Shapiro-Wilk-Test (Shapiro und Wilk 1965)

$$
W=\frac{b^{2}}{(n-1) s^{2}}
$$

Man geht davon aus, dass bei tatsächlicher Normalverteilung beide Varianzen annähernd identisch sind. Bei großer Diskrepanz zwischen geschätzter und tatsächlicher Varianz muss von einer fehlenden Normalverteilung ausgegangen werden (Shapiro und Wilk 1965).

Der Shapiro-Wilk-Test wurde in dieser Arbeit zur Überprüfung der Normalverteilung bei kontinuierlichen Variablen angewendet. Für diese klinische Studie ergab sich dabei keine Normalverteilung. Deshalb wurde im weiteren Verlauf der Wilcoxon-Rangsummentest verwendet.

\subsubsection{Wilcoxon-Rangsummentest}

Der Wilcoxon-Rangsummentest gehört zu den nicht-parametrischen Verfahren der Statistik. Normalverteilte Variablen werden mittels t-Test überprüft, wohingegen nicht normalverteilte Parameter mittels Wilcoxon-Rangsummentest überprüft werden.

Der nichtparametrische Test dient der Überprüfung, ob zwei unabhängige Stichproben derselben Grundgesamtheit angehören. Er untersucht beispielsweise auf einen Unterschied z. B. im Mittelwert oder Median (Wilcoxon 1945). 
Die Berechnungen dieses Tests basieren auf einer Rangbildung. Die einzelnen Daten der beiden zu untersuchenden Patientengruppen (Katheter vs. kein Katheter) werden in einer Tabelle notiert. Eine beispielhafte Berechnung findet sich in Tabelle 1.

Tabelle 1 Beispielhafte Berechnung des Wilcoxon-Rangsummentests

\begin{tabular}{c|c|c|c} 
& Katheter & kein Katheter & Differenz \\
\hline $\begin{array}{c}\text { Krankenhausaufenthalt } \\
\text { Patient 1 }\end{array}$ & 5 & 9 & $\mathbf{- 4}$ \\
\hline $\begin{array}{c}\text { Krankenhausaufenthalt } \\
\text { Patient 2 }\end{array}$ & 4 & 5 & $\mathbf{- 1}$ \\
\hline $\begin{array}{c}\text { Krankenhausaufenthalt } \\
\text { Patient 3 }\end{array}$ & 7 & 3 & $\mathbf{+ 4}$
\end{tabular}

Anschließend bildet man eine Differenz zwischen den einzelnen Werten.

Aufgrund dieser Differenz wird die Rangfolge festgelegt (Wilcoxon 1945). Die Vorzeichen der Differenz werden ebenfalls notiert. Für die positiven Ränge und die negativen Ränge wird jeweils die Summe gebildet. Die kleinere Summe der Ränge wird anschließend weiterverwendet um den Erwartungswert $u_{w}$ der Nullhypothese zu berechnen. $\mathrm{N}$ ist dabei die Anzahl der Paardifferenzen ungleich 0 (Schwarz und Bruderer Enzler 2016a).

\section{Formel 2 Berechnung Wilcoxon-Rangsummentest}

$$
\mu_{\mathrm{w}}=\frac{\mathrm{n} \cdot(\mathrm{n}+1)}{4}
$$

Anschließend wird dieser Wert durch weitere Berechnungen auf Signifikanz überprüft.

Im Rahmen dieser Arbeit wurde dieser Test angewendet, um nicht normalverteilte kontinuierliche Variablen zwischen den unterschiedlichen Patientengruppen zu vergleichen.

\subsubsection{Chi-Quadrat-Test}

Der Chi-Quadrat-Test ( $x^{2}$-Test) oder auch Chi-Quadrat-Homogenitätstest wird verwendet, um einen Zusammenhang zwischen zwei kategorialen Variablen zu errechnen (Du Prel et al. 2010). Hierbei untersucht man den Zusammenhang mehrerer Variablen auf ein Merkmal X (Pearson 2009). Die Variablen dieses Tests 
sind in Tabelle 6 dargestellt (z. B. Alter, Geschlecht, Katheterintervention etc.). Das Merkmal X stellt den Endpunkt Krankenhausaufenthalt in Tagen dar. Man berechnet mittels der nachfolgenden Formel die Teststatistik $\mathrm{X} 2$.

Formel 3 Berechnung Teststatistik Chi-Quadrat (Schwarz und Bruderer Enzler 2016b)

$$
\chi^{2}=\sum_{j=1}^{k} \frac{\left(h_{b j}-h_{e j}\right)^{2}}{h_{e j}}
$$

k stellt die Anzahl der Kategorien (Katheter und kein Katheter) dar, $\mathrm{H}_{\mathrm{bj}}$ die Anzahl der beobachteten und $\mathrm{H}_{\mathrm{ej}}$ die erwartete Häufigkeit dar (Schwarz und Bruderer Enzler 2016b).

Um den berechneten Wert auf Signifikanz zu überprüfen, sucht man in zugehörigen Chi-Quadrat-Verteilungstabellen (Pearson 2009) den kritischen Wert. Wenn der Wert der Teststatistik größer ist als der kritische Wert ist das Ergebnis signifikant (Schwarz und Bruderer Enzler 2016b).

Der Chi-Quadrat-Test wurde in dieser Studie zur Herstellung einer Verbindung zwischen den Variablen verwendet. Die Ergebnisse finden sich in Tabelle 4 der Männer und Tabelle 10 der Frauen.

\subsubsection{Statistische Modelle}

\subsubsection{Lineare Regression}

Die lineare Regression stellt einen statistischen Zusammenhang zwischen zwei Variablen mathematisch dar (Fahrmeir et al. 2009).

Man untersucht den Zusammenhang zwischen einer unabhängigen Variable (Katheterintervention) und einer abhängigen Variable (Krankenhausaufenthaltsdauer) und modelliert eine lineare Funktion durch die Formel

$$
Y 1=a+B^{*} x i
$$

\section{Formel 4 lineare Regression}

- $a=$ Achsenabschnitt

- $B=$ Steigung

- $Y=$ abhängige Variable

- $\quad x=$ unabhängige Variable 
Alternativ kann durch eine Regressionsgerade in einer Grafik [Abbildung 7] die lineare Beziehung hergestellt werden (Draper und Smith 1998).

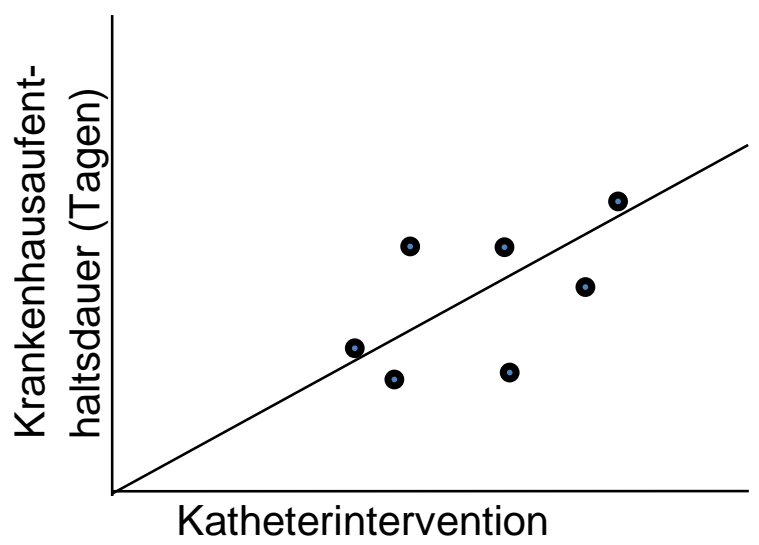

\section{Abbildung 7 Beispiel grafische Darstellung einer linearen Regression}

Man kann bei der linearen Regression zwischen einer einfachen und einer multiplen Regression, je nachdem ob man von einer unabhängigen Variable oder mehreren unabhängigen Variablen ausgeht, differenzieren.

In dieser klinischen Studie erfolgten lineare uni- und multivariable Regressionsanalysen für den primären Endpunkt Krankenhausaufenthalt in Tagen.

Alle linearen Regressionsmodelle wurden auf folgende Annahmen überprüft:

> Lineare Beziehung zwischen abhängigen und unabhängigen Variablen

$>\quad$ Normalverteilung der Residuen

$>\quad$ Erwartungswert des Fehlerterms beträgt 0

> Homoskedastizität bzw. gleichbleibende Varianz der Residuen

$>\quad$ Unabhängigkeit der Residuen

$>$ Keine (perfekte) Multikollinearität zwischen unabhängigen Variablen (Varianzinflationsfaktor (VIF) < 2)

$>\quad$ Normalverteilung der Residuen

\subsubsection{Univariates Regressionsmodell}

Zunächst wurden sämtliche Variablen in univariaten Regressionsmodellen betrachtet. Hierbei wurde die Krankenhausaufenthaltsdauer in Bezug auf eine mögliche Katheteranlage untersucht. 
Nur Variablen, die kleiner als das Signifikanzniveau alpha $<0,05$ waren, wurden in die multivariable Analyse aufgenommen.

\subsubsection{Multivariables Regressionsmodell}

In der multivariablen Regressionsanalyse wurden mehrere unabhängige Variablen untersucht, die Einfluss auf die Zielvariable haben könnten. Hierfür kann man entweder mathematisch mittels einer Formel oder grafisch einen Zusammenhang darstellen.

Für die klinische Studie wurde deshalb in multivariablen Modellen der Einfluss einer Katheteranlage auf die Krankenhausaufenthaltsdauer ermittelt, adjustiert für univariat signifikante Variablen sowie solche Variablen, die als klinische StörgröBen (Confounder) definiert wurden.

\subsection{Confounder}

Confounder sind Störgrößen, bei denen davon ausgegangen wird, dass sie sowohl die unabhängige als auch die abhängige Variable beeinflussen (Miettinen 1974; Valente et al. 2017). Um bei der Effektschätzung in statistischen Modellen den störenden Einfluss auszuschalten, ist eine Adjustierung für bekannte Confounder angezeigt.

Als Confounder wurden das Alter der Patienten bei Diagnosestellung, sowie die Diagnose von Fieber unmittelbar vor oder während des Krankenhausaufenthalts erachtet. Im klinischen Alltag wird bei Fieber aber auch bei Patienten mit einem höheren Alter häufiger ein Katheter gelegt. Gleichzeitig kann aufgrund klinischer Erfahrung davon ausgegangen werden, dass ältere Patienten im Durchschnitt einen längeren Krankenhausaufenthalt haben und das Fieber ebenfalls den Krankenhausaufenthalt verlängert. Die Daten wurden auf Basis der Patientenanamnese oder der gemessenen Körpertemperatur erhoben. 
Abbildung 8 zeigt die vermutete Beziehung zwischen Confoundern und Expositions- sowie Zielvariablen.

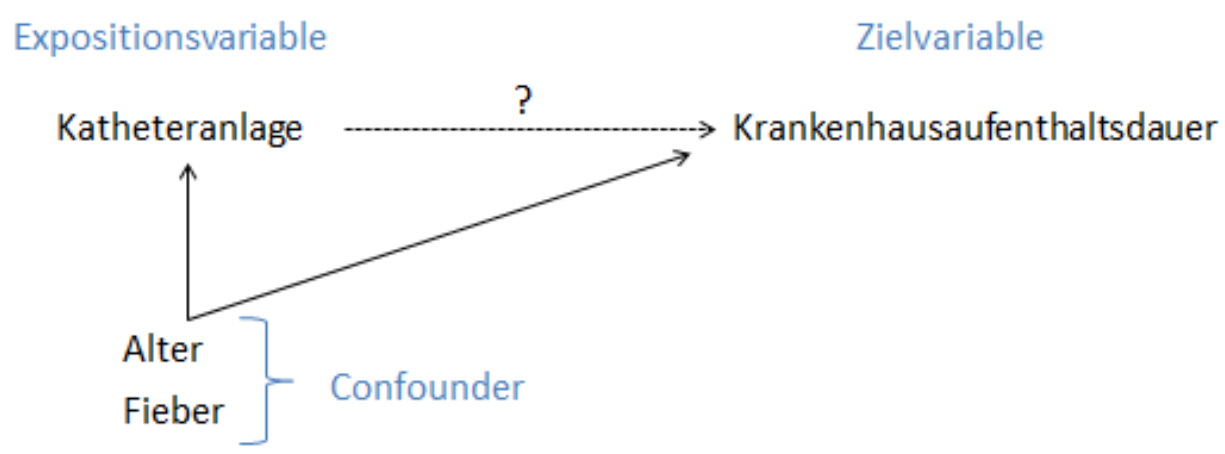

\section{Abbildung 8 Confounder}

Das Schaubild (directed acyclic graph) demonstriert, wie Alter und Fieber möglicherweise einen Confounder für die Beziehung zwischen der primären Expositionsvariablen „Katheteranlage“ und dem Endpunkt „Krankenhausaufenthalt" darstellen. Es ist zum Beispiel denkbar, dass ältere Patienten mit einer größeren Wahrscheinlichkeit einen Katheter erhalten und dass ältere Patienten eine grundsätzlich längere Aufenthaltsdauer haben. Damit wäre der beobachtete Effekt, dass eine Katheteranlage mit einer längeren Krankenhausaufenthaltsdauer einhergeht, zumindest teilweise auf das Patientenalter zurückzuführen. 


\section{Darstellung der Ergebnisse}

\subsection{Prospektive Ergebnisse}

Wie bereits anfangs erwähnt, wurde die ursprünglich nur als prospektive Studie ausgelegte klinische Studie aufgrund eines zu kleinen Patientenkollektivs durch eine retrospektive Betrachtung ersetzt. In diesem Kapitel werden die einzelnen Ursachen für das zu kleine Patientenkollektiv kurz betrachtet sowie die erfassten Daten des Qol-Fragebogens und der VAS dargestellt.

\subsection{1 Übersicht Patientenkollektiv Männer}

Die ursprünglich geplante Anzahl der zu untersuchenden Patienten konnte aufgrund unterschiedlicher Einflüsse nicht erreicht werden. Es stellten sich unerwartet weniger Patienten (lediglich 30 Patienten) mit Prostatitis oder Epididymitis in der UMG vor als angenommen. Erschwerend kam hinzu, dass von diesen 30 Patienten lediglich fünf Patienten hätten eingeschlossen werden können. Diese Patienten wiesen alle Kriterien für den Einschluss in die Studie auf, konnten vom ärztlichen Personal aber nicht eingeschlossen werden. Die anderen Patienten konnten aufgrund unterschiedlicher Gründe nicht miterfasst werden. Lediglich ein Patient konnte eingeschlossen und komplett inklusive Follow-Up erfasst werden. Die einzelnen Patienten sind in der nachfolgenden Tabelle 2 aufgelistet.

Tabelle 2 Männliches Patientenkollektiv der prospektiven Studie

\begin{tabular}{cc}
\hline $\begin{array}{c}\text { Patienten die seit 1. August 2016 (während der Studienzeit) } \\
\text { behandelt wurden }\end{array}$ & 30 \\
\hline Patienten mit Kontraindikationen für einen SPK & 12 \\
\hline Bei Aufnahme aus Indikation heraus Katheter bekommen & 7 \\
\hline Incompliance & 2 \\
\hline Studie abgelehnt & 1 \\
\hline Eugestimmt, aber wieder aus Studie ausgeschlossen & 1 \\
\hline Eorngeschlossen und erfasst & 1 \\
\hline Vorzeitig selbst entlassen & 1 \\
\hline
\end{tabular}




\subsubsection{Ergebnisse Qol-Fragebogen und VAS}

Da lediglich der eine männliche Patient, der komplett in die Studie eingeschlossen werden konnte, den Qol-Fragebogen und die VAS ausgefüllt hat, muss auf die Auswertung der ursprünglich geplanten Fragebögen verzichtet werden.

\subsection{2 Übersicht Patientenkollektiv Frauen}

Bei den Frauen mit Pyelonephritis stellten sich während der laufenden Studie lediglich 16 Patientinnen in der urologischen Klinik der UMG vor. Davon wiesen 7 Patientinnen keine Kontraindikationen auf und hätten somit vom ärztlichen Personal eingeschlossen werden können. 5 Patientinnen konnten vollständig in die prospektive Studie eingeschlossen werden. Die genauen Angaben der Patienten finden sich in Tabelle 3.

Tabelle 3 Weibliches Patientenkollektiv der prospektiven Studie

\begin{tabular}{cc}
\hline $\begin{array}{c}\text { Patienten seit 1. August 2016 in der UMG mit der Diagnose } \\
\text { „akute Pyelonephritis“ }\end{array}$ & 16 \\
\hline In Studie eingeschlossen & 5 \\
\hline Studie abgelehnt & 1 \\
\hline Durch Indikation bereits Katheter bekommen & 3 \\
\hline
\end{tabular}

\subsubsection{Ergebnisse Qol-Fragebogen / VAS}

Aufgrund des zu kleinen Patientenkollektivs von 5 eingeschlossenen Frauen konnte der Quality of Life-Fragebogen nicht adäquat ausgewertet werden. Auch die Visuelle Analogskala konnte aufgrund des zu geringen Patientenkollektivs nicht adäquat ausgewertet werden. 


\subsection{Retrospektive Ergebnisse}

Im folgenden Kapitel werden die Ergebnisse der retrospektiven Studie vorgestellt. Dabei wird das männliche sowie weibliche Patientenkollektiv getrennt voneinander betrachtet und anschließend kurz in einem gesamten Kollektiv bewertet.

\subsubsection{Männer}

\subsubsection{Deskriptive Statistik}

In die retrospektive klinische Studie wurden 50 männliche Patienten eingeschlossen, von denen 34 Patienten einen Katheter bekommen hatten.

Von den 34 männlichen Patienten mit Katheterableitung erhielten 7 Patienten einen SPK und 27 einen transurethralen Katheter bei Aufnahme. Von den 27 Patienten mit transurethraler Harnableitung hatten 5 Patienten durch unzureichende klinische Besserung im Anschluss einen SPK erhalten.

16 Patienten hatten während ihres Krankenhausaufenthaltes keinen Katheter bekommen.

Aufgrund des kleinen Patientenkollektivs wurde für die explorative Statistik lediglich die Frage, ob ein Katheter gelegt wurde berücksichtigt, unabhängig der Art des Katheters.

Die einzelnen Mittelwerte der erfassten Parameter sowie die zugehörigen $p-W e r t e$ sind in nachfolgender Tabelle 4 ausführlich dargestellt. Signifikante p-Werte wurden zur übersichtlicheren Darstellung hervorgehoben.

Tabelle 4 Übersicht aller Parameter der männlichen Studienpatienten

\begin{tabular}{lcccc}
\hline & $\begin{array}{c}\text { Beide } \\
\text { Gruppen } \\
(\mathbf{n}=50)\end{array}$ & $\begin{array}{c}\text { Kein } \\
\text { Katheter } \\
(\mathbf{n = 1 6 )}\end{array}$ & Katheter & p-Wert \\
& $(\mathbf{n = 3 4 )}$ & \\
\hline Geschlecht & $50(100 \%)$ & $16(100 \%)$ & $34(100 \%)$ & \\
Männlich & $59( \pm 16)$ & $49( \pm 19)$ & $64( \pm 11)$ & $\mathbf{0 , 0 0 2}$ \\
\hline Alter & & & & $\mathbf{0 , 0 1 3}$ \\
\hline Alter dichotomisiert & & & &
\end{tabular}




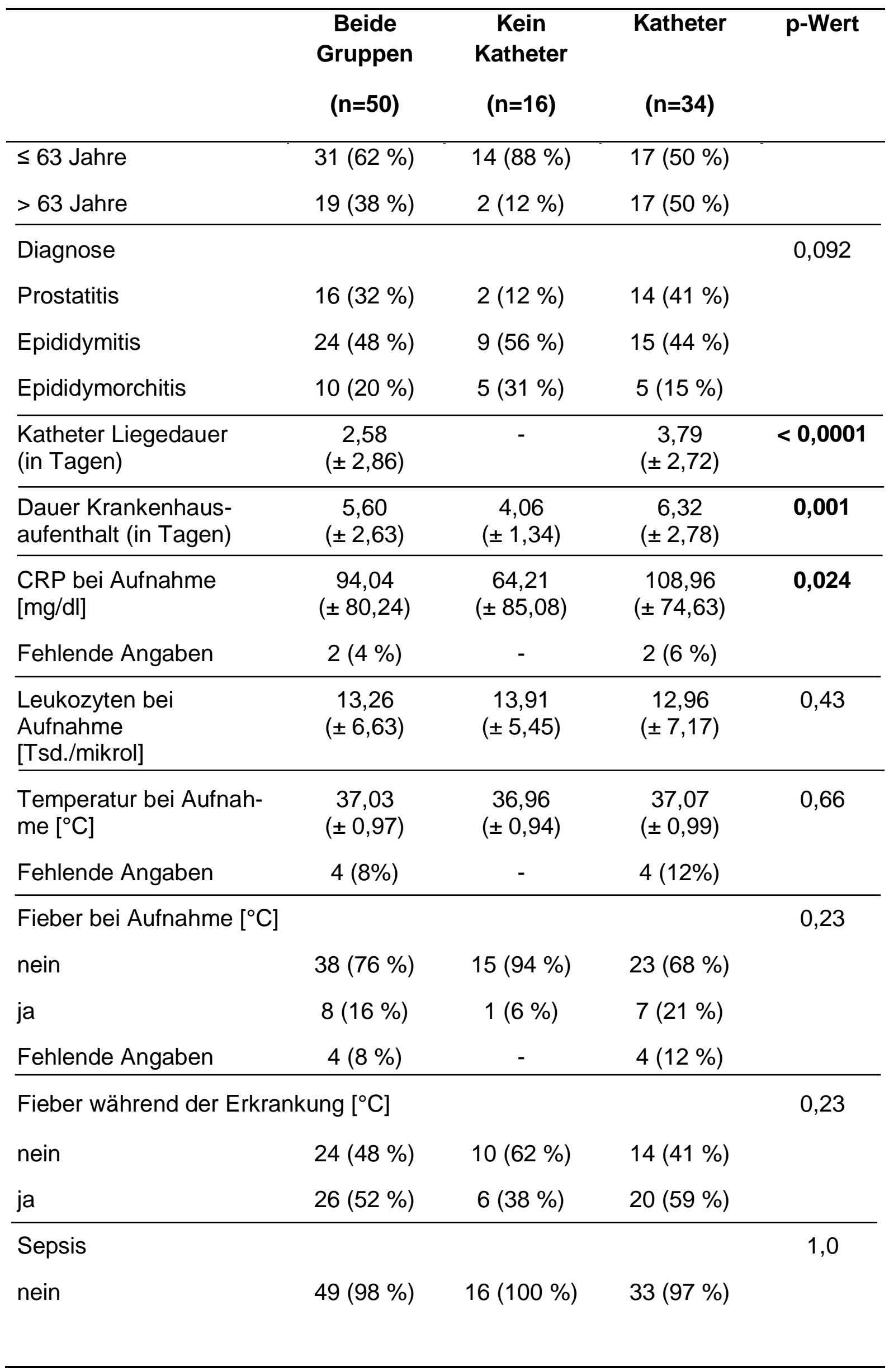




\begin{tabular}{|c|c|c|c|c|}
\hline & $\begin{array}{c}\text { Beide } \\
\text { Gruppen } \\
(n=50)\end{array}$ & $\begin{array}{c}\text { Kein } \\
\text { Katheter } \\
(n=16)\end{array}$ & $\begin{array}{c}\text { Katheter } \\
(n=34)\end{array}$ & p-Wert \\
\hline \multicolumn{5}{|l|}{ Sepsis } \\
\hline ja & $1(2 \%)$ & - & $1(3 \%)$ & \\
\hline Urinkultur bei Aufnahme & & & & 0,79 \\
\hline negativ & $21(42 \%)$ & $7(44 \%)$ & $14(41 \%)$ & \\
\hline E. coli & $19(38 \%)$ & $5(31 \%)$ & $14(41 \%)$ & \\
\hline andere & $10(20 \%)$ & $4(25 \%)$ & $6(18 \%)$ & \\
\hline Verwendetes Antibiotikum & & & & 0,01 \\
\hline Cephalosporine & $3(6 \%)$ & - & $3(9 \%)$ & \\
\hline Fluorchinolone & $29(58 \%)$ & $15(94 \%)$ & $14(41 \%)$ & \\
\hline Carbapeneme & $3(6 \%)$ & $1(6 \%)$ & $2(6 \%)$ & \\
\hline Beta-Lactame & $2(4 \%)$ & - & $2(6 \%)$ & \\
\hline AB gewechselt & $5(10 \%)$ & - & $5(15 \%)$ & \\
\hline andere & $8(16 \%)$ & - & $8(24 \%)$ & \\
\hline $\mathrm{AB}$ resistenzgerecht & & & & 0,043 \\
\hline nein & $8(16 \%)$ & - & $8(24 \%)$ & \\
\hline ja & $42(84 \%)$ & $16(100 \%)$ & $26(76 \%)$ & \\
\hline Antibiotikumwechsel & & & & 0,16 \\
\hline AB gewechselt & $5(10 \%)$ & - & $5(15 \%)$ & \\
\hline nicht gewechselt & $45(90 \%)$ & $16(100 \%)$ & $29(85 \%)$ & \\
\hline Diabetes mellitus & & & & 0,074 \\
\hline nein & $44(88 \%)$ & $12(75 \%)$ & $32(94 \%)$ & \\
\hline ja & $6(12 \%)$ & $4(25 \%)$ & $2(6 \%)$ & \\
\hline Hypertonie & & & & 1,0 \\
\hline nein & $38(76 \%)$ & $12(75 \%)$ & $26(76 \%)$ & \\
\hline ja & $12(24 \%)$ & $4(25 \%)$ & $8(24 \%)$ & \\
\hline
\end{tabular}




\begin{tabular}{|c|c|c|c|c|}
\hline & $\begin{array}{c}\text { Beide } \\
\text { Gruppen } \\
(n=50)\end{array}$ & $\begin{array}{c}\text { Kein } \\
\text { Katheter } \\
(n=16)\end{array}$ & $\begin{array}{c}\text { Katheter } \\
(n=34)\end{array}$ & p-Wert \\
\hline Maligne Erkrankungen & & & & 1,0 \\
\hline nein & $39(78 \%)$ & $13(81 \%)$ & $26(76 \%)$ & \\
\hline ja & $11(22 \%)$ & $3(19 \%)$ & $8(24 \%)$ & \\
\hline Charlson Comorbidity Inder & & & & 0,086 \\
\hline 0 & $9(18 \%)$ & $5(31 \%)$ & $4(12 \%)$ & \\
\hline 1 & $11(22 \%)$ & $6(38 \%)$ & $5(15 \%)$ & \\
\hline 2 & $11(22 \%)$ & $2(12 \%)$ & $9(26 \%)$ & \\
\hline 3 & $4(8 \%)$ & - & $4(12 \%)$ & \\
\hline 4 & $8(16 \%)$ & $1(6 \%)$ & $7(21 \%)$ & \\
\hline 5 & $3(6 \%)$ & - & $3(9 \%)$ & \\
\hline 6 & $2(4 \%)$ & $1(6 \%)$ & $1(3 \%)$ & \\
\hline 7 & $1(2 \%)$ & $1(6 \%)$ & - & \\
\hline 8 & $1(2 \%)$ & - & $1(3 \%)$ & \\
\hline Charlson Comorbidity Index & dichotomisiert & & & 0,006 \\
\hline Hoch $(\geq 2)$ & $30(60 \%)$ & $5(31 \%)$ & $25(74 \%)$ & \\
\hline Niedrig $(<2)$ & $20(40 \%)$ & $11(69 \%)$ & $9(26 \%)$ & \\
\hline
\end{tabular}

\subsubsection{Univariate Analyse}

Zunächst wurde analysiert, welche Variablen statistisch signifikante Prädiktoren für die Krankenhausaufenthaltsdauer sind, um diese anschließend in multivariablen Analysen zu betrachten. Hierbei wurden nur Variablen mit $p<0,05$ als signifikant betrachtet.

In Tabelle 5 sind die Ergebnisse der univariaten Analyse detailliert dargestellt. 
Tabelle 5 Univariate Analyse der Männer

$\begin{array}{cccc}\begin{array}{c}\text { Punktschätzer } \\ \text { Änderung } \\ \text { Kranken- }\end{array} & \begin{array}{c}\text { Unteres 95\% } \\ \text { Konfidenz- } \\ \text { intervall }(\mathrm{KI})\end{array} & \begin{array}{c}\text { Oberes } 95 \% \\ \text { Konfidenz- } \\ \text { intervall }(\mathrm{KI})\end{array} & \mathrm{p} \text {-Wert } \\ \text { hausaufent- } & & \\ \text { haltsdauer } & & \\ \text { [in Tagen] } & & \end{array}$

\begin{tabular}{|c|c|c|c|c|}
\hline \\
\hline \multicolumn{5}{|l|}{$\frac{\text { Diagnose }}{\text { Prostatitis }}$} \\
\hline Epididymitis & $-0,10$ & $-1,79$ & 1,58 & 0,904 \\
\hline Epididymorchitis & $-0,81$ & $-2,92$ & 1,29 & 0,453 \\
\hline $\begin{array}{l}\text { Fieber bei } \\
\text { Aufnahme }\end{array}$ & 0,43 & $-1,52$ & 2,38 & 0,670 \\
\hline $\begin{array}{l}\text { Fieber während des } \\
\text { Krankheitsverlaufes }\end{array}$ & 0,75 & $-0,70$ & 2,21 & 0,316 \\
\hline Sepsis ja & 1,43 & $-3,81$ & 6,67 & 0,595 \\
\hline Urinkultur E. coli & 0,70 & $-0,95$ & 2,34 & 0,409 \\
\hline $\begin{array}{l}\text { Urinkultur } \\
\text { andere }\end{array}$ & 0,96 & $-1,04$ & 2,95 & 0,352 \\
\hline$\frac{\text { Antibiotikum }}{\text { Fluorchinolone }}$ & $-2,25$ & $-4,87$ & 0,36 & 0,098 \\
\hline Carbapeneme & 0,33 & $-3,19$ & 3,85 & 0,854 \\
\hline Beta-Lactame & $-1,17$ & $-5,10$ & 2,77 & 0,564 \\
\hline $\begin{array}{l}\text { Antibiotikum wäh- } \\
\text { rend Therapie ge- } \\
\text { wechselt }\end{array}$ & $-0,47$ & $-3,62$ & 2,68 & 0,773 \\
\hline Weitere Antibiotika & 1,96 & $-0,96$ & 4,88 & 0,195 \\
\hline $\begin{array}{l}\text { Resistenzgerechte } \\
\text { Antibiotikatherapie }\end{array}$ & $-3,60$ & $-5,33$ & $-1,87$ & $<0,001$ \\
\hline Diabetes mellitus & $-1,44$ & $-3,67$ & 0,79 & 0,211 \\
\hline $\begin{array}{l}\text { Maligne Erkrankun- } \\
\text { gen }\end{array}$ & 0,63 & $-1,14$ & 2,4 & 0,488 \\
\hline Charlson niedrig <2 & $-1,58$ & $-3,02$ & $-0,15$ & 0,035 \\
\hline Katheter & 2,26 & 0,82 & 3,70 & 0,003 \\
\hline $\begin{array}{l}\text { Antibiotikum nicht } \\
\text { gewechselt }\end{array}$ & $-0,67$ & $-3,11$ & 1,78 & 0,595 \\
\hline
\end{tabular}




\begin{tabular}{lcccc}
\hline & $\begin{array}{c}\text { Punktschätzer } \\
\text { Änderung } \\
\text { Kranken- } \\
\text { hausaufent- } \\
\text { haltsdauer } \\
\text { [in Tagen] }\end{array}$ & $\begin{array}{c}\text { Unteres 95\% } \\
\text { Konfidenz- } \\
\text { intervall (KI) }\end{array}$ & $\begin{array}{c}\text { Oberes 95\% } \\
\text { Konfidenz- } \\
\text { intervall (KI) }\end{array}$ & p-Wert \\
\hline \hline $\begin{array}{l}\text { Liegedauer Katheter } \\
\text { [in Tagen] }\end{array}$ & 0,53 & 0,32 & 0,74 & $<0,001$ \\
\hline $\begin{array}{l}\text { Leukozytenwert bei } \\
\text { Aufnahme } \\
\text { [Tsd./mikrol] }\end{array}$ & 0,05 & $-0,06$ & 0,16 & 0,395 \\
\hline $\begin{array}{l}\text { Temperatur bei } \\
\left.\text { Aufnahme [ }{ }^{\circ} \mathrm{C}\right]\end{array}$ & $-0,14$ & $-0,92$ & 0,64 & 0,725 \\
\hline $\begin{array}{l}\text { Charlson Comorbi- } \\
\text { dity Index }\end{array}$ & 0,38 & 0,03 & 0,74 & $\mathbf{0 , 0 4 1}$ \\
\hline $\begin{array}{l}\text { CRP bei Aufnahme } \\
\text { [mg/dl] }\end{array}$ & 0,01 & 0,00 & 0,02 & $\mathbf{0 , 0 1 5}$ \\
\hline \begin{tabular}{l} 
Alter \\
\hline
\end{tabular} & 0,05 & 0,01 & 0,1 & $\mathbf{0 , 0 2 4}$ \\
\hline
\end{tabular}

\subsubsection{Multivariable Analyse}

In der anschließend durchgeführten multivariablen Analyse wurde der Endpunkt Krankenhausaufenthalt in Tagen unter Berücksichtigung der Intervention (Katheter vs. kein Katheter) untersucht.

Die Nullhypothese bleibt „Die Anlage eines Katheters führt im Vergleich zu einem Verzicht auf die Katheteranlage zu keinem Unterschied in der Krankenhausaufenthaltsdauer in Tagen".

Außerdem wurden weitere unabhängige, aber im univariaten Modell statistisch signifikante Variablen $(p<0,05)$ in die Berechnung mit einbezogen.

Hierzu gehören:

$>$ Teilgesamtheit Alter (> 63 Jahre, $\leq 63$ Jahre)

> Fieber während des Krankheitsverlaufes

$>$ Resistenzgerechte Antibiotikagabe

> CRP bei Aufnahme 
Als Confounder agieren in diesem Modell, wie in Abbildung 8 dargestellt, das Alter und Fieber während des Krankenhausaufenthaltes, da man davon ausgehen kann, dass Fieber und Alter den Krankenhausaufenthalt verlängern.

Nachfolgende Tabelle 6 zeigt das multivariable lineare Regressionsmodel ohne Interaktionsterm.

Tabelle 6 Lineares multivariables Modell ohne Interaktionsterm

\begin{tabular}{|c|c|c|c|c|}
\hline Variable & $\begin{array}{l}\text { Punktschätzer } \\
\text { Änderung } \\
\text { Krankenhaus- } \\
\text { aufenthalts- } \\
\text { dauer [in Tage] }\end{array}$ & $\begin{array}{l}\text { Unteres } \\
95 \% \\
\text { Konfidenz- } \\
\text { intervall }\end{array}$ & $\begin{array}{c}\text { Oberes } \\
95 \% \\
\text { Konfidenz- } \\
\text { intervall }\end{array}$ & $p$-Wert \\
\hline \multicolumn{5}{|l|}{$\begin{array}{l}\text { Keine Katheteranlage } \\
\text { (Referenzkategorie, } \\
\text { Ref.) }\end{array}$} \\
\hline Katheteranlage & 0,80 & $-0,64$ & 2,24 & 0,281 \\
\hline \multicolumn{5}{|l|}{ Alter $\leq 63$ Jahre (Ref.) } \\
\hline Alter > 63 Jahre & 1,46 & 0,18 & 2,74 & 0,030 \\
\hline \multicolumn{5}{|l|}{$\begin{array}{l}\text { Kein Fieber im } \\
\text { Krankheitsverlauf } \\
\text { (Ref.) }\end{array}$} \\
\hline $\begin{array}{l}\text { Fieber im Krankheits- } \\
\text { verlauf }\end{array}$ & 0,02 & $-1,19$ & 1,23 & 0,972 \\
\hline \multicolumn{5}{|l|}{$\begin{array}{l}\text { Nicht resistenzge- } \\
\text { rechte Antibiotika- } \\
\text { therapie (Ref.) }\end{array}$} \\
\hline $\begin{array}{l}\text { Resistenzgerechte } \\
\text { Antibiotikatherapie }\end{array}$ & $-3,03$ & $-4,63$ & $-1,44$ & 0,001 \\
\hline $\begin{array}{l}\text { CRP bei Aufnahme } \\
{[\mathrm{mg} / \mathrm{dll}]}\end{array}$ & 0,01 & $<0,01$ & 0,02 & 0,048 \\
\hline
\end{tabular}

In nachfolgender Tabelle 7 ist das Ergebnis des linearen Modelles mit Interaktionsterm detailliert dargestellt. 
Tabelle 7 Multivariable Analyse mit Interaktionsterm

\begin{tabular}{|c|c|c|c|c|}
\hline Variable & $\begin{array}{l}\text { Punktschätzer } \\
\text { Änderung } \\
\text { Krankenhaus- } \\
\text { aufenthaltsdauer } \\
\text { [in Tage] }\end{array}$ & $\begin{array}{l}\text { Unteres } \\
95 \% \\
\text { Konfidenz- } \\
\text { intervall }\end{array}$ & $\begin{array}{l}\text { Oberes } \\
95 \% \\
\text { Konfidenz- } \\
\text { intervall }\end{array}$ & $p$-Wert \\
\hline \multicolumn{5}{|l|}{$\begin{array}{l}\text { Keine Katheter- } \\
\text { anlage (Ref.) }\end{array}$} \\
\hline Katheteranlage & 1,36 & 0,06 & 2,66 & 0,047 \\
\hline \multicolumn{5}{|l|}{$\begin{array}{l}\text { Alter } \leq 63 \text { Jahre } \\
\text { (Ref.) }\end{array}$} \\
\hline Alter $>63$ Jahre & 5,45 & 3,04 & 7,87 & $<0,001$ \\
\hline \multicolumn{5}{|l|}{$\begin{array}{l}\text { Kein Fieber im } \\
\text { Krankheitsverlauf } \\
\text { (Ref.) }\end{array}$} \\
\hline $\begin{array}{l}\text { Fieber im Krank- } \\
\text { heitsverlauf }\end{array}$ & $-0,25$ & $-1,32$ & 0,82 & 0,650 \\
\hline \multicolumn{5}{|l|}{$\begin{array}{l}\text { Nicht resistenzge- } \\
\text { rechte Antibiotika- } \\
\text { therapie (Ref.) }\end{array}$} \\
\hline $\begin{array}{l}\text { Resistenzgerechte } \\
\text { Antibiotikatherapie }\end{array}$ & $-0,40$ & $-2,39$ & 1,59 & 0,69 \\
\hline $\begin{array}{l}\text { CRP bei Aufnahme } \\
{[\mathrm{mg} / \mathrm{dl}]}\end{array}$ & 0,01 & $<0,001$ & 0,01 & 0,042 \\
\hline \multicolumn{5}{|l|}{$\begin{array}{l}\text { Alter } \leq 63 \text { und keine } \\
\text { resistenzgerechte } \\
\text { Antibiotikatherapie } \\
\text { (Ref.) }\end{array}$} \\
\hline $\begin{array}{l}\text { Alter }>63 \text { und resis- } \\
\text { tenzgerechte Antibi- } \\
\text { otikatherapie }\end{array}$ & $-5,06$ & $-7,77$ & $-2,35$ & 0,001 \\
\hline
\end{tabular}


In der multivariablen Analyse haben Männer, die einen Katheter bei Aufnahme erhalten haben, im Vergleich zu Männern ohne Katheteranlage einen um 1,36 Tage $(95 \% \mathrm{KI} 0,06$ bis 2,66) statistisch signifikant verlängerten Krankenhausaufenthalt $(p-$ Wert $=0,047)$.

Ebenfalls zeigt sich, dass eine Erhöhung des CRP-Spiegels bei Aufnahme um eine Einheit mit einem um 0,01 Tage verlängerten Krankenhausaufenthalt einhergeht $(95 \% \mathrm{KI}<0,001$ bis 0,013$)$. Mit einem $\mathrm{p}$-Wert von 0,042 ist dieses Ergebnis ebenfalls statistisch signifikant.

Des Weiteren zeigte sich eine statistisch signifikante Interaktion zwischen Patientenalter und Anlage eines Katheters ( $p$-Wert $<0,001$ ). Hieraus lässt sich ableiten, dass der Effekt einer Katheteranlage bei jungen und alten Patienten einen unterschiedlichen Einfluss hat. Daher schlossen sich weitere Subgruppenanalysen des männlichen Kollektivs hinsichtlich des Alters an.

\subsubsection{Outcome Krankenhausaufenthalt Männer > 63 Jahre $(n=19)$}

Das Gesamtkollektiv wurde zur genaueren Betrachtung in zwei Subgruppen unterteilt: Männer $\leq 63$ Jahre und $>63$ Jahre. In der nachfolgenden Tabelle 8 ist die Subgruppenanalyse für Männer $>63$ Jahre dargestellt.

Tabelle 8 Subgruppenanalyse der Männer > 63 Jahre

\begin{tabular}{ccccc}
\hline & Punktschätzer & Unteres & Oberes & p-Wert \\
Änderung & $95 \%$ & $95 \%$ & \\
Kariable & Krankenhaus- & Konfidenz- & Konfidenz- \\
& aufenthaltsdauer & intervall & intervall & \\
& [in Tage] & &
\end{tabular}

Keine Katheteranlage (Ref.)

\begin{tabular}{lllll} 
Katheteranlage & 0,18 & $-2,52$ & 2,87 & 0,900 \\
\hline $\begin{array}{l}\text { Kein Fieber im } \\
\text { Krankheitsverlauf } \\
\text { (Ref.) }\end{array}$ & & & & \\
$\begin{array}{l}\text { Fieber im Krank- } \\
\text { heitsverlauf }\end{array}$ & $-0,34$ & $-2,21$ & 1,52 & 0,721 \\
\end{tabular}




\begin{tabular}{lcccc}
\hline Variable & $\begin{array}{c}\text { Punktschätzer } \\
\text { Änderung } \\
\text { Krankenhaus- } \\
\text { aufenthaltsdauer } \\
\text { [in Tage] }\end{array}$ & $\begin{array}{c}\text { Unteres } \\
95 \% \\
\text { Konfidenz- } \\
\text { intervall }\end{array}$ & $\begin{array}{c}\text { Oberes } \\
95 \% \\
\text { Konfidenz- } \\
\text { intervall }\end{array}$ & p-Wert \\
\hline $\begin{array}{l}\text { Nicht resistenzge- } \\
\text { rechte Antibiotika- } \\
\text { therapie (Ref.) }\end{array}$ & & & & \\
$\begin{array}{l}\text { Resistenzgerechte } \\
\text { Antibiotikatherapie }\end{array}$ & $-5,67$ & $-8,10$ & $-3,23$ & $<0,001$ \\
\hline $\begin{array}{l}\text { CRP-Werte bei } \\
\text { Aufnahme [mg/dl] }\end{array}$ & 0,010 & $-0,002$ & 0,022 & 0,133 \\
\hline
\end{tabular}

In der Subgruppenanalyse der Männer > 63 Jahre zeigte sich, dass sie bei resistenzgerechter Antibiotikagabe eine statistisch signifikante $(p-$ Wert $<0,001)$ verkürzte Krankenhausaufenthaltsdauer von 5,67 Tagen (95\% KI -8,10 bis -3,23) aufweisen im Vergleich zu > 63-Jährigen mit nicht resistenzgerechter Antibiotikagabe.

Für die Krankenhausaufenthaltsdauer der Männer > 63 Jahre ergibt sich mit Katheteranlage keine statistisch signifikante Änderung ( $p$ - Wert $=0,900)$. Die Anlage eines Katheters hat in dieser Subgruppe also keinen Einfluss auf die Krankenhausaufenthaltsdauer.

Außerdem muss festgehalten werden, dass der im Gesamtkollektiv beobachtete Effekt des CRP-Wertes bei Aufnahme in dem Subgruppenkollektiv vermutlich powerbedingt (Kollektivgröße 22 Männer) nicht nachgewiesen werden kann.

\subsubsection{Outcome Krankenhausaufenthalt Männer $\leq 63$ Jahre $(n=31)$}

In der nachfolgenden Tabelle 9 sind die Ergebnisse der Subgruppenanalyse der Männer $\leq 63$ Jahre abgebildet. 
Tabelle 9 Subgruppenanalyse der Männer $\leq 63$ Jahre

\begin{tabular}{|c|c|c|c|c|}
\hline Variable & $\begin{array}{c}\text { Punktschätzer } \\
\text { Änderung Kran- } \\
\text { kenhausaufent- } \\
\text { haltsdauer } \\
\text { [in Tage] }\end{array}$ & $\begin{array}{c}\text { Unteres } 95 \% \\
\text { Konfidenz- } \\
\text { intervall }\end{array}$ & $\begin{array}{c}\text { Oberes } 95 \% \\
\text { Konfidenz- } \\
\text { intervall }\end{array}$ & p-Wert \\
\hline \multicolumn{5}{|l|}{$\begin{array}{l}\text { Keine Katheter- } \\
\text { anlage (Ref.) }\end{array}$} \\
\hline Katheteranlage & 2,0 & 1,81 & 5,48 & 0,009 \\
\hline \multicolumn{5}{|l|}{$\begin{array}{l}\text { Kein Fieber im } \\
\text { Krankheitsverlauf } \\
\text { (Ref.) }\end{array}$} \\
\hline $\begin{array}{l}\text { Fieber im Krank- } \\
\text { heitsverlauf }\end{array}$ & $-0,38$ & $-1,64$ & 0,88 & 0,563 \\
\hline \multicolumn{5}{|l|}{$\begin{array}{l}\text { Nicht resistenzge- } \\
\text { rechte Antibiotika- } \\
\text { therapie (Ref.) }\end{array}$} \\
\hline $\begin{array}{l}\text { Resistenzgerechte } \\
\text { Antibiotikatherapie }\end{array}$ & $-0,04$ & $-1,69$ & 0,88 & 0,563 \\
\hline $\begin{array}{l}\text { CRP bei Aufnah- } \\
\mathrm{me}[\mathrm{mg} / \mathrm{dl}]\end{array}$ & 0,01 & $-0,002$ & 0,01 & 0,167 \\
\hline
\end{tabular}

In dieser Subgruppenanalyse zeigte sich, dass jüngere Männer $\leq 63$ Jahre, die einen Katheter bei Aufnahme erhalten haben, einen statistisch signifikanten $(p-$ Wert $=0,009)$ verlängerten Krankenhausaufenthalt um 2,0 Tage $(95 \% \mathrm{KI} 1,81$ bis 5,48$)$ aufweisen im Vergleich zu denen ohne Katheter.

Der für die Erhöhung des CRP-Spiegels bei Aufnahme beobachtete Effekt ist auch hier, vermutlich powerbedingt (Kollektivgröße 28 Männer), nicht statistisch signifikant $(p-$ Wert $=0,167)$. 


\subsubsection{Frauen}

\subsubsection{Deskriptive Statistik}

In dem weiblichen Patientenkollektiv wurden ebenfalls 50 Studienteilnehmer erfasst, von denen 15 Patientinnen einen Katheter und 35 Patientinnen keinen Katheter bekommen hatten. Die mittlere Liegedauer des Katheters lag bei 4,4 Tagen (SD 2,27 Tage).

In der nachfolgenden Tabelle 10 sind die einzelnen Verteilungen der Parameter, die Mittelwerte sowie $p$ - Werte ausführlich dargestellt.

Tabelle 10 Übersicht aller Parameter der weiblichen Studienpatienten

\begin{tabular}{|c|c|c|c|c|}
\hline & $\begin{array}{l}\text { Beide } \\
\text { Gruppen } \\
(n=50)\end{array}$ & $\begin{array}{c}\text { Kein } \\
\text { Katheter } \\
(n=15)\end{array}$ & $\begin{array}{l}\text { Katheter } \\
(n=35)\end{array}$ & $p$-Wert \\
\hline Geschlecht & & & & 1,0 \\
\hline Weiblich & $50(100 \%)$ & $15(100 \%)$ & $35(100 \%)$ & \\
\hline Alter & $35( \pm 19)$ & $28( \pm 15)$ & $37( \pm 20)$ & 0,12 \\
\hline Alter dichotomisiert & & & & 0,65 \\
\hline$\leq 63$ Jahre & $44(88 \%)$ & $14(93 \%)$ & $30(86 \%)$ & \\
\hline$>63$ Jahre & $6(12 \%)$ & $1(7 \%)$ & $5(14 \%)$ & \\
\hline Diagnose & & & & 0,13 \\
\hline Pyelonephritis rechts & $19(38 \%)$ & $9(60 \%)$ & $10(29 \%)$ & \\
\hline Pyelonephritis links & $21(42 \%)$ & $4(27 \%)$ & $17(49 \%)$ & \\
\hline Pyelonephritis beidseits & $10(20 \%)$ & $2(13 \%)$ & $8(23 \%)$ & \\
\hline $\begin{array}{l}\text { Katheter Liegedauer } \\
\text { [in Tagen] }\end{array}$ & $\begin{array}{c}3,08 \\
( \pm 2,78) \\
\end{array}$ & - & $\begin{array}{c}4,40 \\
( \pm 2,27)\end{array}$ & $<0,0001$ \\
\hline $\begin{array}{l}\text { Dauer Krankenhaus- } \\
\text { aufenthalt [ in Tagen] }\end{array}$ & $\begin{array}{c}5,74 \\
( \pm 2,30) \\
\end{array}$ & $\begin{array}{c}5,07 \\
( \pm 2,12) \\
\end{array}$ & $\begin{array}{c}6,03 \\
( \pm 2,35) \\
\end{array}$ & 0,23 \\
\hline $\begin{array}{l}\text { CRP bei Aufnahme } \\
\text { [mg/dl] }\end{array}$ & $\begin{array}{c}113,494 \\
( \pm 115,52)\end{array}$ & $\begin{array}{c}59,82 \\
( \pm 83,42)\end{array}$ & $\begin{array}{c}136,5 \\
( \pm 122,0)\end{array}$ & 0,009 \\
\hline $\begin{array}{l}\text { Leukozyten bei Auf- } \\
\text { nahme } \\
\text { [Tsd./mikrol] }\end{array}$ & $\begin{array}{c}13,51 \\
( \pm 6,50)\end{array}$ & $\begin{array}{c}10,7 \\
( \pm 4,89)\end{array}$ & $\begin{array}{c}14,71 \\
( \pm 6,79)\end{array}$ & 0,012 \\
\hline
\end{tabular}




\begin{tabular}{|c|c|c|c|c|}
\hline & $\begin{array}{l}\text { Beide } \\
\text { Gruppen } \\
(n=50)\end{array}$ & $\begin{array}{c}\text { Kein } \\
\text { Katheter } \\
(n=15)\end{array}$ & $\begin{array}{l}\text { Katheter } \\
(\mathrm{n}=35)\end{array}$ & $\mathrm{p}$-Wert \\
\hline $\begin{array}{l}\text { Temperatur bei Auf- } \\
\text { nahme }\left[{ }^{\circ} \mathrm{C}\right]\end{array}$ & $\begin{array}{c}37,38 \\
( \pm 1,06)\end{array}$ & $\begin{array}{c}37,15 \\
( \pm 0,81)\end{array}$ & $\begin{array}{c}37,48 \\
( \pm 1,15)\end{array}$ & 0,56 \\
\hline Fieber bei Aufnahme $\left[{ }^{\circ} \mathrm{C}\right]$ & & & & 0,20 \\
\hline nein & $32(64 \%)$ & $12(80 \%)$ & $20(57 \%)$ & \\
\hline ja & $18(36 \%)$ & $3(20 \%)$ & $15(43 \%)$ & \\
\hline Fieber während der Erkran & ung $\left[{ }^{\circ} \mathrm{C}\right]$ & & & 0,011 \\
\hline nein & $13(26 \%)$ & $8(53 \%)$ & $5(14 \%)$ & \\
\hline ja & $37(74 \%)$ & $7(47 \%)$ & $30(86 \%)$ & \\
\hline Sepsis & & & & 1,0 \\
\hline nein & $47(94 \%)$ & $14(93 \%)$ & $33(94 \%)$ & \\
\hline ja & $3(6 \%)$ & $1(7 \%)$ & $2(6 \%)$ & \\
\hline Urinkultur bei Aufnahme & & & & 0,79 \\
\hline negativ & $19(38 \%)$ & $5(33 \%)$ & $14(40 \%)$ & \\
\hline E. coli & $19(38 \%)$ & $7(47 \%)$ & $12(34 \%)$ & \\
\hline andere & $12(24 \%)$ & $3(20 \%)$ & $9(26 \%)$ & \\
\hline Antibiotikum & & & & 0,42 \\
\hline Cephalosporine & $20(40 \%)$ & $7(47 \%)$ & $13(37 \%)$ & \\
\hline Fluorchinolone & $14(28 \%)$ & $5(33 \%)$ & $9(26 \%)$ & \\
\hline Carbapeneme & - & - & - & \\
\hline Beta-Lactame & $2(4 \%)$ & - & $2(6 \%)$ & \\
\hline AB gewechselt & $10(20 \%)$ & $1(7 \%)$ & $9(26 \%)$ & \\
\hline andere & $4(8 \%)$ & $2(13 \%)$ & $2(6 \%)$ & \\
\hline AB resistenzgerecht & & & & 0,14 \\
\hline nein & $11(22 \%)$ & $1(7 \%)$ & $10(29 \%)$ & \\
\hline ja & $39(78 \%)$ & $14(93 \%)$ & $25(71 \%)$ & \\
\hline Antibiotikumwechsel & & & & 0,25 \\
\hline
\end{tabular}




\begin{tabular}{|c|c|c|c|c|}
\hline & $\begin{array}{c}\text { Beide } \\
\text { Gruppen } \\
(n=50)\end{array}$ & $\begin{array}{c}\text { Kein } \\
\text { Katheter } \\
(n=15)\end{array}$ & $\begin{array}{l}\text { Katheter } \\
(n=35)\end{array}$ & $p$-Wert \\
\hline AB gewechselt & $10(20 \%)$ & $1(7 \%)$ & $9(26 \%)$ & \\
\hline nicht gewechselt & $40(80 \%)$ & $14(93 \%)$ & $26(74 \%)$ & \\
\hline Diabetes mellitus & & & & 1,0 \\
\hline nein & $49(98 \%)$ & $15(100 \%)$ & $34(97 \%)$ & \\
\hline ja & $1(2 \%)$ & - & $1(3 \%)$ & \\
\hline Hypertonie & & & & 1,0 \\
\hline nein & $46(92 \%)$ & $14(93 \%)$ & $32(91 \%)$ & \\
\hline ja & $4(8 \%)$ & $1(7 \%)$ & $3(9 \%)$ & \\
\hline Maligne Erkrankung & & & & 1,0 \\
\hline nein & $48(96 \%)$ & $15(100 \%)$ & $33(94 \%)$ & \\
\hline ja & $2(4 \%)$ & - & $2(6 \%)$ & \\
\hline \multicolumn{2}{|c|}{ Charlson Comorbidity Index } & & & 0,88 \\
\hline 0 & $36(72 \%)$ & $13(87 \%)$ & $23(66 \%)$ & \\
\hline 1 & $2(4 \%)$ & - & $2(6 \%)$ & \\
\hline 2 & $3(6 \%)$ & - & $3(9 \%)$ & \\
\hline 3 & $2(4 \%)$ & $1(7 \%)$ & $1(3 \%)$ & \\
\hline 4 & $1(2 \%)$ & - & $1(3 \%)$ & \\
\hline 5 & $2(4 \%)$ & - & $2(6 \%)$ & \\
\hline 6 & $2(4 \%)$ & $1(7 \%)$ & $1(3 \%)$ & \\
\hline 9 & $1(2 \%)$ & - & $1(3 \%)$ & \\
\hline 10 & $1(2 \%)$ & - & $1(3 \%)$ & \\
\hline \multicolumn{3}{|c|}{ Charlson Comorbidity Index dichotomisiert } & & 0,30 \\
\hline Hoch $(\geq 2)$ & $12(24 \%)$ & $2(13 \%)$ & $10(29 \%)$ & \\
\hline Niedrig $(<2)$ & $38(76 \%)$ & $13(87 \%)$ & $25(71 \%)$ & \\
\hline
\end{tabular}




\subsubsection{Univariate Analyse}

Zunächst wurde auch bei dem Patientenkollektiv der 50 Frauen analysiert, welche Variablen statistisch signifikante Prädiktoren für die Krankenhausaufenthaltsdauer sind.

Variablen mit einem $p-$ Wert $<0,05$ in den univariaten Analysen wurden für einen Einschluss in die multivariable Analyse in Betracht gezogen.

In nachfolgender Tabelle 11 ist das Ergebnis der univariaten Analyse der Frauen dargestellt.

Tabelle 11 Univariate Analyse der Frauen

\begin{tabular}{lcccc}
\hline & $\begin{array}{c}\text { Punktschätzer } \\
\text { Änderung } \\
\text { Krankenhaus- } \\
\text { aufenthalts- } \\
\text { dauer } \\
\text { [in Tagen] }\end{array}$ & $\begin{array}{c}\text { Unteres 95\% } \\
\text { Konfidenz- } \\
\text { intervall }\end{array}$ & $\begin{array}{c}\text { Oberes 95\% } \\
\text { Konfidenz- } \\
\text { intervall }\end{array}$ & p- Wert \\
\hline Diagnose & 0,94 & $-0,49$ & 2,37 & 0,21 \\
Pyelonephritis links & 0,94 & $-0,82$ & 2,71 & 0,30 \\
\hline Pyelonephritis beidseits & 0,06 & $-1,28$ & 1,40 & 0,93 \\
\hline Fieber bei Aufnahme & 0,48 & $-0,98$ & 1,94 & 0,52 \\
\hline Fieber während des & 0,63 & $-2,08$ & 3,34 & 0,65 \\
Krankheitsverlaufes & $-1,11$ & $-2,51$ & 0,30 & 0,13 \\
\hline Sepsis & 0,89 & $-0,71$ & 2,48 & 0,28 \\
\hline Urinkultur E. coli & & & & \\
Urinkultur andere & $-0,96$ & $-2,47$ & 0,55 & 0,220 \\
\hline Antibiotikum & 1,90 & $-1,31$ & 5,11 & 0,252 \\
Fluorchinolone & 1,00 & $-0,68$ & 2,68 & 0,249 \\
Beta-Lactame & 1,65 & $-0,72$ & 4,02 & 0,180 \\
Antibiotikum während & $-1,38$ & $-2,89$ & 0,12 & 0,078 \\
\hline $\begin{array}{l}\text { Therapie gewechselt } \\
\text { Weitere Antibiotika }\end{array}$ & 4,35 & $-0,09$ & 8,78 & 0,061 \\
$\begin{array}{l}\text { Resistenzgerechte An- } \\
\text { tibiotikatherapie }\end{array}$ & & & &
\end{tabular}




\begin{tabular}{lcccc}
\hline & $\begin{array}{c}\text { Punktschätzer } \\
\text { Änderung } \\
\text { Krankenhaus- } \\
\text { aufenthalts- } \\
\text { dauer } \\
\text { [in Tagen] }\end{array}$ & $\begin{array}{c}\text { Unteres 95\% } \\
\text { Konfidenz- } \\
\text { intervall }\end{array}$ & $\begin{array}{c}\text { Oberes 95\% } \\
\text { Konfidenz- } \\
\text { intervall }\end{array}$ & p- Wert \\
\hline Maligne Erkrankungen & 4,44 & 1,40 & 7,48 & $\mathbf{0 , 0 0 6}$ \\
\hline Charlson niedrig <2 & $-2,1$ & $-3,48$ & $-0,71$ & $\mathbf{0 , 0 0 5}$ \\
\hline Katheter & 0,96 & $-0,42$ & 2,34 & 0,178 \\
\hline $\begin{array}{l}\text { Antibiotikum nicht ge- } \\
\text { wechselt }\end{array}$ & $-1,08$ & $-2,66$ & 0,51 & 0,189 \\
\hline Liegedauer Katheter & 0,52 & 0,33 & 0,70 & $<\mathbf{0 , 0 0 1}$ \\
\hline $\begin{array}{l}\text { Leukozyten bei Auf- } \\
\text { nahme [Tsd./mikrol] }\end{array}$ & 0,20 & 0,12 & 0,29 & $<\mathbf{0 , 0 0 1}$ \\
\hline $\begin{array}{l}\text { Liegedauer Katheter [in } \\
\text { Tagen] }\end{array}$ & 0,52 & 0,33 & 0,70 & $<\mathbf{0 , 0 0 1}$ \\
\hline $\begin{array}{l}\text { Temperatur bei Auf- } \\
\text { nahme [ }{ }^{\circ} \mathrm{C} \text { ] }\end{array}$ & $-0,14$ & $-0,75$ & 0,47 & 0,649 \\
\hline $\begin{array}{l}\text { Charlson Comorbidity } \\
\text { Index }\end{array}$ & 0,40 & 0,15 & 0,65 & $\mathbf{0 , 0 0 3}$ \\
\hline $\begin{array}{l}\text { CRP bei Aufnahme } \\
\text { [mg/dl] }\end{array}$ & 0,01 & 0,004 & 0,01 & $\mathbf{0 , 0 0 1}$ \\
\hline \begin{tabular}{l} 
Alter \\
\hline
\end{tabular} & 0,03 & $-0,004$ & 0,06 & $\mathbf{0 , 0 9 1}$ \\
\hline
\end{tabular}

\subsubsection{Multivariable Analyse}

In der anschließend auch bei den weiblichen Patientinnen durchgeführten multivariablen Analyse wurde das Outcome Krankenhausaufenthalt in Tagen unter Berücksichtigung der Intervention (Katheter vs. kein Katheter) untersucht.

Die Nullhypothese bleibt „Die Anlage eines Katheters führt im Vergleich zu einem Verzicht auf die Katheteranlage zu keinem Unterschied in der Krankenhausaufenthaltsdauer in Tagen“.

Außerdem wurden weitere unabhängige, aber im univariaten Modell statistisch signifikante Variablen $(p-$ Wert $<0,05)$ in die Berechnung mit einbezogen. 
Hierzu gehörten:

$>$ Alter

$>$ Fieber während des Krankheitsverlaufes

$>$ Leukozyten bei Aufnahme

$>$ Charlson Comorbidity Index

Als Confounder interagiert in dem Modell der Frauen ebenfalls das Alter und Fieber während des Krankenhausaufenthalts.

In der nachfolgenden Tabelle 12 sind die Ergebnisse der multivariablen Analyse des Gesamtkollektivs der Frauen dargestellt.

Tabelle 12 Ergebnisse Outcome Krankenhausaufenthalt der Frauen

\begin{tabular}{|c|c|c|c|c|}
\hline Variable & $\begin{array}{l}\text { Punktschätzer } \\
\text { Änderung } \\
\text { Krankenhaus- } \\
\text { aufenthalts- } \\
\text { dauer } \\
\text { [in Tagen] }\end{array}$ & $\begin{array}{l}\text { Unteres } 95 \% \\
\text { Konfidenz- } \\
\text { intervall }\end{array}$ & $\begin{array}{l}\text { Oberes } 95 \% \\
\text { Konfidenz- } \\
\text { intervall }\end{array}$ & $p$-Wert \\
\hline \multicolumn{5}{|l|}{$\begin{array}{l}\text { Keine Katheter- } \\
\text { anlage (Ref.) }\end{array}$} \\
\hline Katheteranlage & 0,31 & $-0,89$ & 1,51 & 0,614 \\
\hline Alter & $-0,02$ & $-0,05$ & 0,02 & 0,403 \\
\hline \multicolumn{5}{|l|}{$\begin{array}{l}\text { Kein Fieber im } \\
\text { Krankheitsverlauf } \\
\text { (Ref.) }\end{array}$} \\
\hline $\begin{array}{l}\text { Fieber im Krank- } \\
\text { heitsverlauf }\end{array}$ & $-0,91$ & $-2,19$ & 0,37 & 0,170 \\
\hline $\begin{array}{l}\text { Leukozyten bei } \\
\text { Aufnahme }\end{array}$ & 0,20 & 0,12 & 0,28 & $<0,001$ \\
\hline \multicolumn{5}{|l|}{ Charlson } \\
\hline $\begin{array}{l}\text { Comorbidity In- } \\
\text { dex }\end{array}$ & 0,42 & 0,13 & 0,70 & 0,006 \\
\hline
\end{tabular}


Für das Gesamtkollektiv der Frauen zeigte sich kein statistisch signifikanter Zusammenhang zwischen der Anlage eines Katheters und der Krankenhausaufenthaltsdauer in Tagen $(p-$ Wert $=0,614)$.

Es besteht auch kein signifikanter Zusammenhang $(p-$ Wert $=0,403)$ zwischen dem Alter der Patienten und dem Krankenhausaufenthalt.

Man konnte aber erkennen, dass eine Erhöhung der Leukozyten bei Aufnahme mit einem statistisch signifikanten ( $p$ - Wert $<0,001)$ verlängerten Krankenhausaufenthalt von 0,20 Tage (95\% Konfidenzintervall 0,12 bis 0,28$)$ pro Einheit Leukozyten (Tsd./mikrol) einhergeht.

Außerdem geht ein um eine Komorbidität höherer Charlson Comorbidity Index mit einem statistisch signifikant $(p-$ Wert $=0,006)$ verlängerten Aufenthalt um 0,42 Tage (95\% Konfidenzintervall 0,13 bis 0,70 ) einher.

\subsubsection{Gemeinsames Patientenkollektiv (Frauen + Männer)}

Bei Betrachtung sämtlicher männlicher und weiblicher Patienten in einem Kollektiv zeigte sich eine statistisch signifikante Interaktion zwischen den Effekten von Geschlecht und Katheteranlage auf die Krankenhausaufenthaltsdauer.

Aufgrund der erschwerten Interpretation einzelner Punktschätzer innerhalb eines statistischen Modells mit Interaktionen wurden daher separate Analysen für Frauen und Männer durchgeführt.

\subsubsection{Veränderte Interventionsvariablen}

\subsubsection{Katheterintervention}

In diesem Kapitel geht es detailliert um den Einfluss der Kathetertypen, die aufgrund des zu kleinen Patientenkollektivs nicht ausreichend in der statistischen Analyse betrachtet werden konnten. 


\subsection{Männer}

Von den 50 männlichen Patienten erhielten 7 Patienten einen SPK bei Aufnahme, 27 einen transurethralen Katheter und 16 Patienten keine Harnableitung.

Von den 27 Patienten mit transurethraler Harnableitung hatten 5 Patienten aufgrund unzureichender Besserung klinischer Beschwerden und eines fehlenden Rückgangs der Entzündungsparameter im Anschluss einen SPK erhalten.

Weiterhin haben 3 Patienten bei unzureichender Besserung nach SPK-Anlage im Verlauf ihres Krankenhausaufenthaltes einen transurethralen Katheter gelegt bekommen. Eine grafische Übersicht über die Verteilung der verschiedenen Kathetertypen im männlichen Patientenkollektiv findet sich in Abbildung 9.

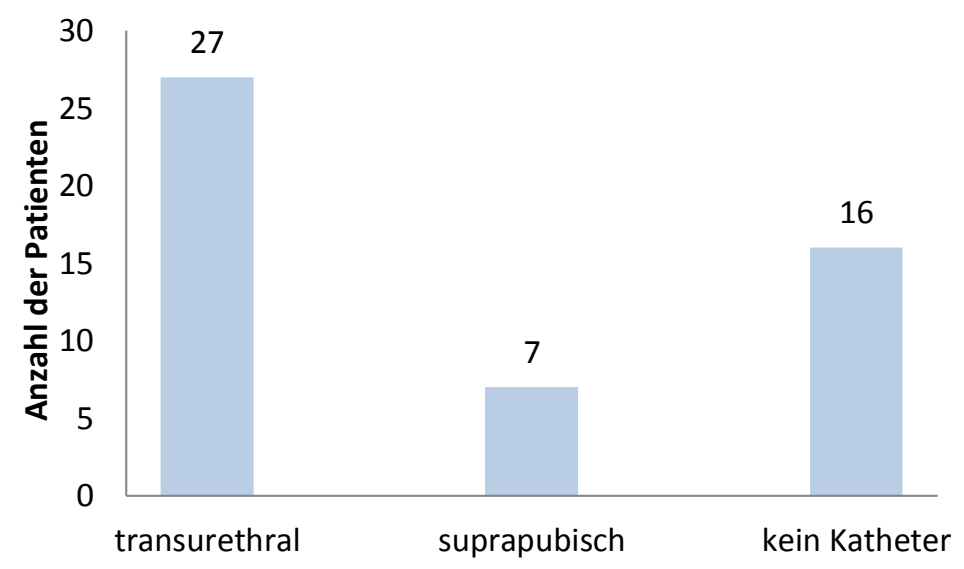

\section{Abbildung 9 Verteilung der Kathetertypen im männlichen Kollektiv}

Interessant ist außerdem die unterschiedliche Liegedauer der verschiedenen Kathetertypen.

Der transurethrale Katheter lag im Mittel 4,42 Tage bei den erfassten 24 Patientendaten (SD 1,613 Tage). Bei 3 Patienten waren keine Angaben zur Katheterliegedauer dokumentiert. Der Median betrug bei Patienten mit transurethralen Katheter 4,5 Tage. Die kürzeste Liegedauer lag bei $1 \mathrm{Tag}$, wohingegen die maximale Liegedauer des transurethralen Katheters 7 Tage betrug.

Bei den 7 Patienten mit SPK konnte lediglich die Liegedauer des Katheters bis zur Entlassung erfasst werden, da der SPK ambulant vom niedergelassenen Urologen entfernt wurde. Die Liegedauer des SPK lag im Mittel bei 4,33 Tagen (SD 2,66 Tage) und wies einen Median von 5 Tagen auf. Die maximale Liegedauer des SPK lag bei 12 Tagen. Die kürzeste Liegedauer lag bei 1 Tag. 
Die beschriebenen Daten sind in nachfolgender Abbildung 10 detailliert dargestellt.

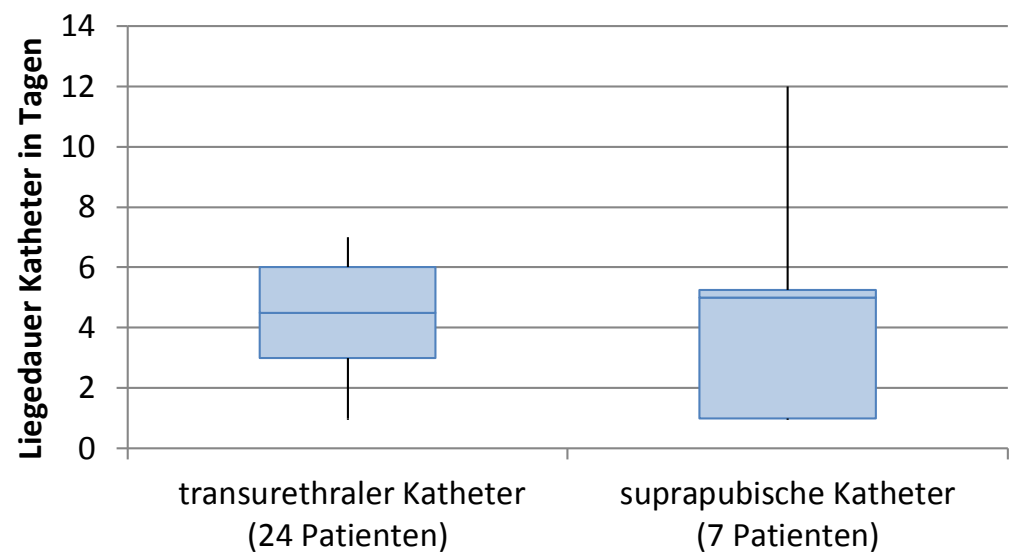

\section{Abbildung 10 Verteilung Liegedauer SPK und DK der Männer}

Außerdem wurde die Krankenhausaufenthaltsdauer der Patienten mit suprapubischem Katheter, mit transurethralem Katheter und fehlender Katheteranlage analysiert.

Patienten ohne Katheteranlage waren im Median 4 Tage hospitalisiert. Die Krankenhausaufenthaltsdauer variiert zwischen dem Minimalwert von 2 bis hin zu einer Dauer von 7 Tagen.

Männer, die bei Aufnahme einen SPK erhalten hatten, waren im Mittel 4,86 Tage im Krankenhaus, bei einer Standardabweichung von 2,67 Tagen. Der Medianwert bei Patienten mit SPK betrug 5 Tage. Die kürzeste Krankenhausaufenthaltsdauer lag bei Patienten mit SPK bei 2 Tagen, der längste Aufenthalt bei 9 Tagen.

Patienten, die einen DK erhalten haben wiesen den längsten Krankenhausaufenthalt von im Mittel 6,70 Tagen auf (SD 2,73 Tage). Die kürzeste Aufenthaltsdauer mit transurethralem Katheter lag bei 3 Tagen, wohingegen die längste Krankenhausaufenthaltsdauer 14 Tage betrug. Eine übersichtliche Darstellung der Krankenhausaufenthaltsdauer findet sich in Abbildung 11. 


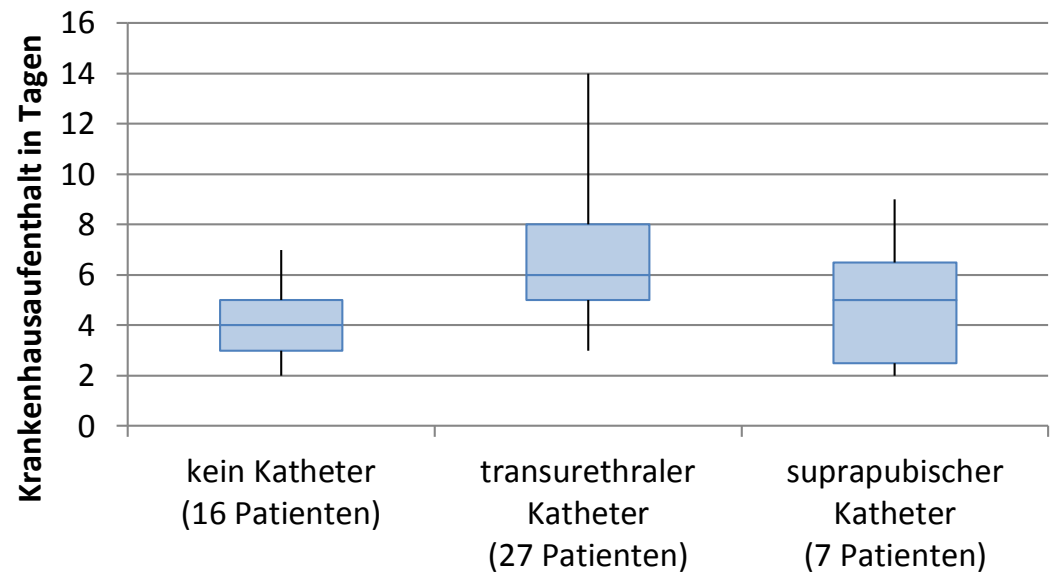

\section{Abbildung 11 Krankenhausaufenthalt der Männer in Kathetergruppen}

Berechnet man allerdings mittels Wilcoxon-Rangsummentest die Auswirkungen der verschiedenen Kathetertypen auf die Krankenhausaufenthaltsdauer ergibt sich für die Krankenhausaufenthaltsdauer keine statistisch signifikante Änderung zwischen einem SPK und einem DK.

Man muss also festhalten, dass der Kathetertyp bei den männlichen Patienten mit Harnableitung keinen statistisch signifikanten Einfluss auf die Krankenhausaufenthaltsdauer ( $p$ - Wert Wilcoxon-Rangsummentest $=0,2021$ ) hat.

\subsection{Frauen}

Bei dem Patientenkollektiv der 50 Frauen erhielten 35 Patientinnen einen transurethralen Katheter, wohingegen 15 Patientinnen ohne Katheter blieben.

Im Mittel lag der transurethrale Katheter bei den Frauen 4,56 Tage, mit einer Standardabweichung von 2,21 Tagen. Bei 2 Patientinnen fehlte die Angabe über die genaue Liegedauer, weshalb diese zur Berechnung nicht berücksichtigt werden konnten.

Die Patientinnen mit Katheter waren im Mittel 6,03 Tage im Krankenhaus (SD 2,35 Tage). Die maximale Krankenhausaufenthaltsdauer bei Patientinnen mit transurethralem Katheter lag bei 11 Tagen. Die kürzeste Aufenthaltsdauer in diesem Patientenkollektiv lag bei 2 Tagen. Der Median für Frauen mit transurethralem Katheter lag bei 6 Tagen. 
Die Frauen ohne Katheteranlage waren lediglich 5,07 Tage im Krankenhaus (SD 2,12 Tage). Die kürzeste Krankenhausaufenthaltsdauer bei Patientinnen ohne Katheter lag bei 2 Tagen, wohingegen der längste Krankenhausaufenthalt bei 9 Tagen lag. Der Median für die Patientinnen ohne Katheteranlage lag bei 5 Tagen. Zur genaueren Betrachtung sind die oben beschriebenen Werte in der Abbildung 12 detailliert dargestellt.

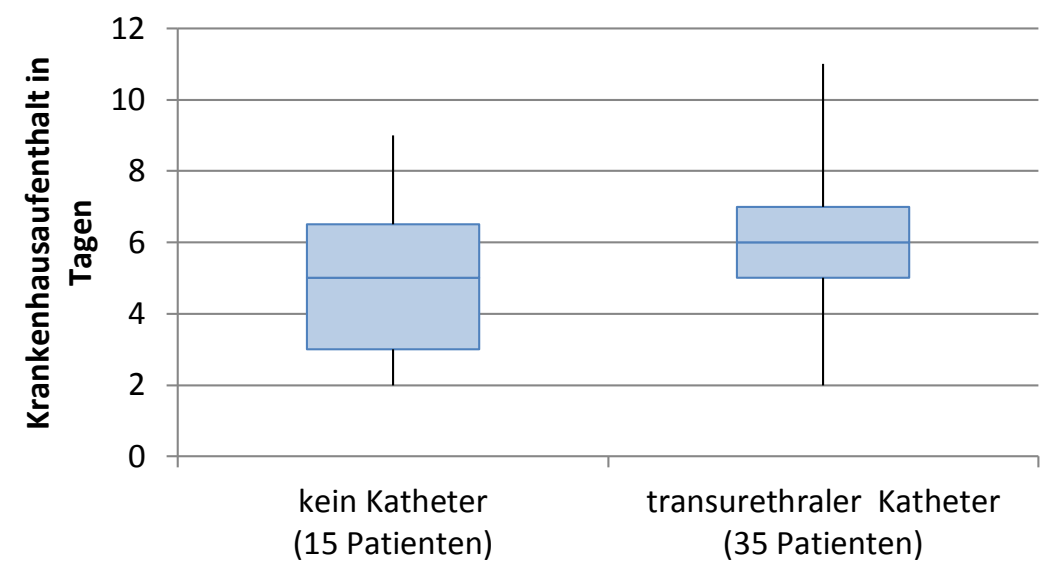

Abbildung 12 Krankenhausaufenthalt der Frauen mit/ohne Katheter 


\subsubsection{Ergebnisse klinischer Parameter}

Männer:

Bei der stationären Aufnahme lag der CRP-Mittelwert aller 50 Patienten bei 94,04 mg/dl (SD 80,24 mg/dl). Der p-Wert für CRP bei Aufnahme, bezogen auf die Krankenhausaufentshaltsdauer in Tagen, betrug 0,02.

Patienten die einen Katheter bekommen hatten, zeigten im Mittel einen CRP-Wert bei Aufnahme von 108,96 mg/dl (SD 74,63 mg/dl), wohingegen Patienten die keinen Katheter bekommen hatten, einen CRP-Wert bei Aufnahme von 64,21 mg/dl aufwiesen (SD 85,08 mg/dl). Dabei fehlten zwei CRP-Wert-Angaben der jüngeren Patienten, weshalb diese beiden Werte als fehlend betrachtet wurden und nicht in die Berechnung mit eingingen.

Betrachtet man bei den Männern die CRP-Werte bei Aufnahme in den beiden Subgruppen, ergibt sich für die jüngeren Männer $\leq 63$ Jahre ein Mittelwert von 78,4 mg/dl (SD 81,35 mg/dl) bei Aufnahme. Der Median für dieses männliche Patientenkollektiv lag bei 69,4 mg/dl. Der niedrigste gemessene CRP-Wert bei Aufnahme lag bei dem jüngeren Kollektiv bei $3 \mathrm{mg} / \mathrm{dl}$, wohingegen der höchste gemessene CRP-Wert bei 297,6 mg/dl lag.

Die Männer > 63 Jahre wiesen dagegen bei Aufnahme einen höheren CRPMittelwert von 112,53 mg/dl (SD 76,62 mg/dl) auf. Der Median dieser Altersgruppe lag bei 117,5 mg/dl. Der niedrigste gemessene CRP-Wert lag in diesem Kollektiv bei $5 \mathrm{mg} / \mathrm{dl}$, der maximale CRP-Wert bei $233,4 \mathrm{mg} / \mathrm{dl}$.

Die einzelnen Werte der beiden Altersgruppen sind in nachfolgender Abbildung 13 dargestellt.

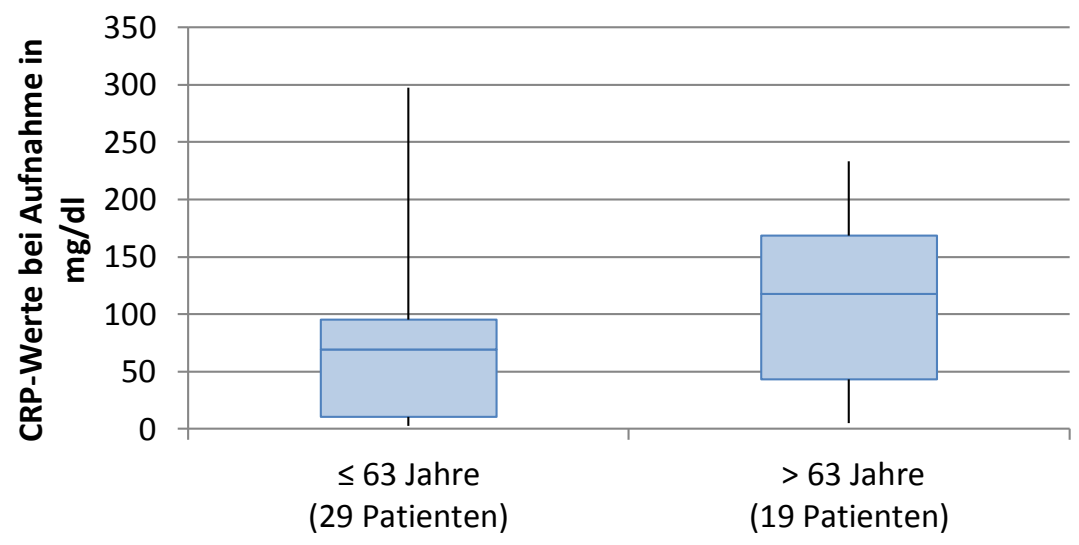


Der Serum-Leukozytenwert lag bei Aufnahme von allen 50 Patienten im Mittel bei 13,26 Tsd./mikrol mit einer SD von 6,63 Tsd./mikrol. Patienten, die einen Katheter bei Aufnahme bekommen hatten, wiesen im Mittel einen Serum-Leukozytenwert von 12,96 Tsd./mikrol (SD 7,17 Tsd./mikrol) bei Aufnahme auf. Patienten ohne Katheteranlage hatten im Mittel einen Leukozytenwert bei Aufnahme von 13,91 Tsd./mikrol, mit einer SD von 5,45 Tsd./mikrol.

Betrachtete man die Leukozytenwerte bei Aufnahme nach dem Alter aufgeschlüsselt ergibt sich für die 31 Männer $\leq 63$ Jahre im Mittel ein Wert von 13,95 Tsd./mikrol (SD 6,41 Tsd./mikrol), wohingegen bei 19 Männern > 63 Jahren bei Aufnahme ein Mittelwert von 12,38 Tsd./mikrol (SD 6,94 Tsd./mikrol) gemessen wurde.

Patienten $\leq 63$ Jahre hatten einen Median von 12,8 Tsd./mikrol Leukozyten bei Aufnahme. Der Minimalwert dieses Kollektivs betrug 4,57 Tsd./mikrol, wohingegen der Maximalwert der Leukozyten bei Aufnahme 27,7 Tsd./mikrol betrug.

Patienten > 63 Jahre hatten einen niedrigeren Median von 11,15 Tsd./mikrol bei Aufnahme. Das gemessene Spektrum der Leukozytenwerte reicht hier von einem Minimalwert von 4,1 Tsd./mikrol Leukozyten bei Aufnahme bis zu einem Maximalwert von 33,8 Tsd./mikrol.

Die grafische Darstellung zu den oberen beschriebenen Leukozytenwerten, aufgeschlüsselt nach dem Patientenalter, findet sich in nachfolgender Abbildung 14.

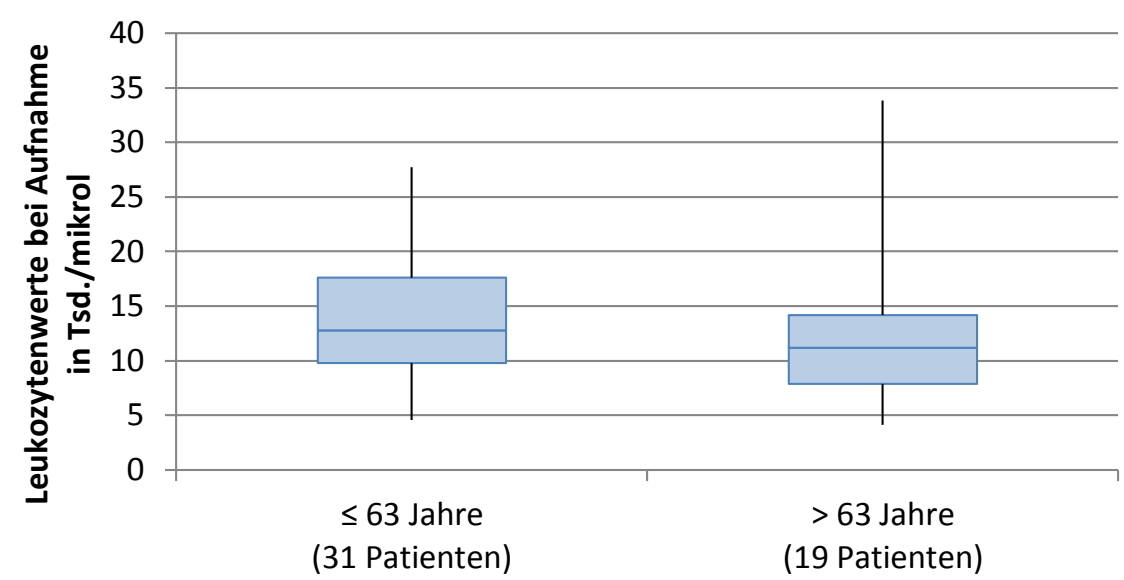

Abbildung 14 Leukozytenwerte Männer bei Aufnahme nach Alter 
Männer, die bei Aufnahme eine Katheteranlage erhalten hatten, wiesen im Mittel eine Körpertemperatur bei Aufnahme von $37,48{ }^{\circ} \mathrm{C}$ auf, wohingegen Patienten ohne Katheteranlage eine Temperatur von $37,15^{\circ} \mathrm{C}$ aufwiesen.

Frauen:

Bei der stationären Aufnahme lag der CRP-Mittelwert aller 50 Frauen bei 113,49 mg/dl, der bei einer Standardabweichung von 115,52 mg/dl nicht aussagekräftig ist. Bei den Frauen, die eine Katheteranlage bei stationärer Aufnahme erhalten hatten, lag der Serum-CRP-Mittelwert bei 136,50 mg/dl (SD 122,00 mg/dl). Der Median dieses Kollektives lag bei $81,8 \mathrm{mg} / \mathrm{dl}$. Die CRP-Werte der Frauen mit Katheteranlage variieren vom Minimalwert $0,7 \mathrm{mg} / \mathrm{dl}$ bis hin zu dem Maximalwert $422,1 \mathrm{mg} / \mathrm{dl}$.

Die Patientinnen, die keinen Katheter bei Aufnahme erhalten hatten, wiesen einen CRP-Mittelwert von 59,82 mg/dl auf (SD 83,42 mg/dl). Der p-Wert für die CRPWerte lag bei 0,01. Der Medianwert der Frauen ohne Katheteranlage lag bei 23,6 mg/dl. Der Minimalwert lag bei Patientinnen ohne Katheteranlage bei $0,2 \mathrm{mg} / \mathrm{dl}$, wohingehen der Maximalwert bei 255,3 mg/dl lag.

Eine ausführliche Darstellung der ermittelten CRP-Werte bei Aufnahme im weiblichen Patientenkollektiv findet sich in Abbildung 15.

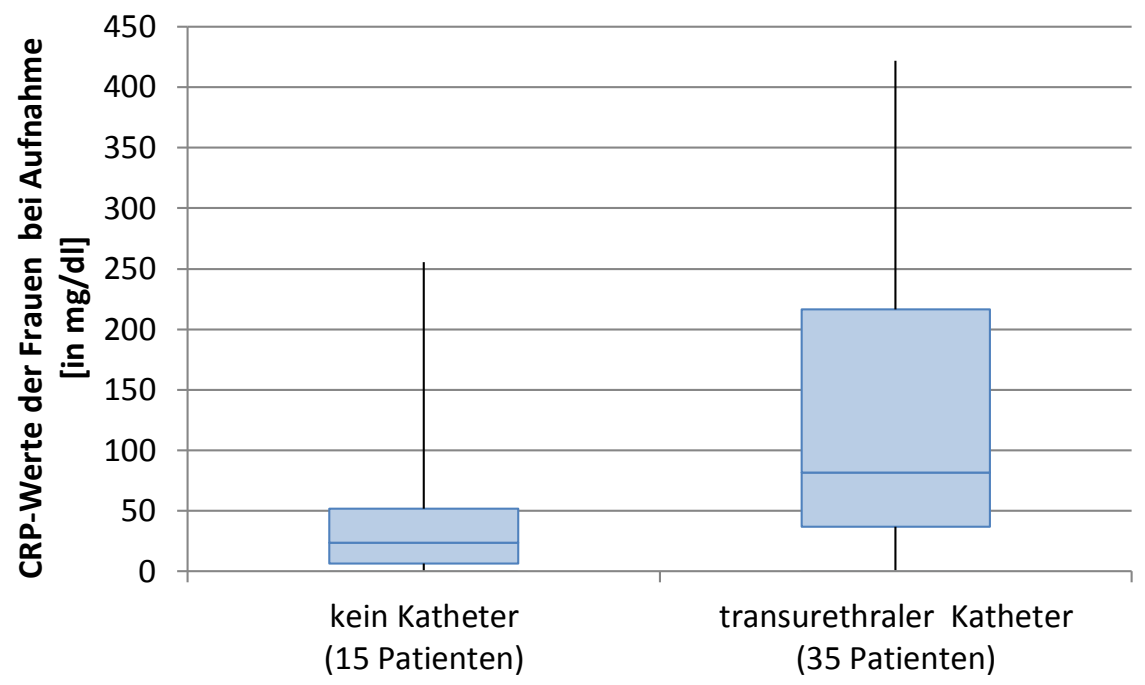

Abbildung 15 CRP-Werte der Frauen bei Aufnahme in Kathetergruppen 
Im gesamten Patientenkollektiv betrug der Mittelwert der Leukozyten bei Aufnahme 13,51 Tsd./mikrol (SD 6,50 Tsd./mikrol).

Patientinnen, die einen Katheter bei Aufnahme erhalten hatten, wiesen einen Mittelwert von 14,71 Tsd./mikrol (SD 6,79 Tsd./mikrol) auf. Die Leukozytenwerte variierten dabei vom Minimum 5,6 Tsd./mikrol bis hin zum Maximum von 41,8 Tsd./mikrol. Der Median dieses Patientenkollektivs mit Katheteranlage lag bei 14,2 Tsd./mikrol.

Patientinnen, die keinen Katheter bei Aufnahme erhalten hatten, wiesen im Mittel einen Leukozytenwert von 10,70 Tsd./mikrol (SD 4,89 Tsd./mikrol) auf. Der Minimalwert lag bei 4,6 Tsd./mikrol, wohingegen der Maximalwert bei 24,5 Tsd./mikrol lag. Der Median betrug bei Patientinnen ohne Katheteranlage 9,8 Tsd./mikrol.

Der p-Wert der Leukozytenwerte bei Aufnahme betrug 0,01. Die oben beschriebenen Werte sind grafisch in Abbildung 16 dargestellt.

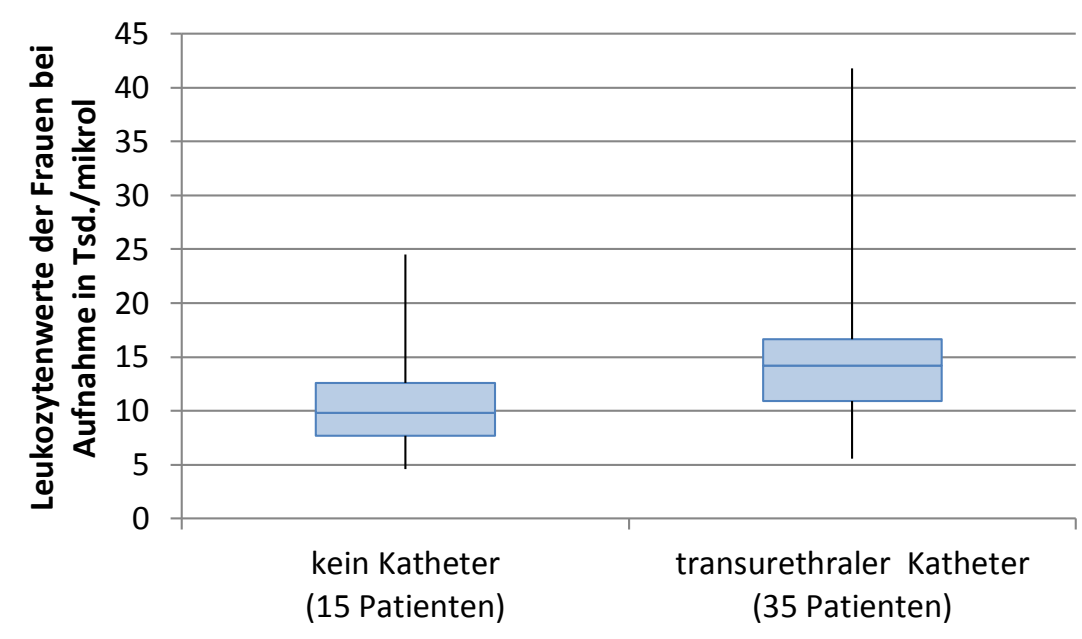

Abbildung 16 Leukozytenwerte der Frauen bei Aufnahme in Kathetergruppen

Außerdem wiesen Frauen, die einen Katheter bei Aufnahme erhalten hatten, eine höhere Körpertemperatur von im Mittel $37,48{ }^{\circ} \mathrm{C}$ auf, wohingegen Patientinnen ohne Katheter im Mittel eine Temperatur von $37,15^{\circ} \mathrm{C}$ aufwiesen. Der zugehörige p-Wert lag bei 0,56 . 


\subsubsection{Ergebnisse der Antibiotikatherapie}

Aufgrund der Verwendung zahlreicher Antibiotika wurde für die statistische Auswertung die Einteilung in „resistenzgerecht verwendetes Antibiotikum“ und „nicht resistenzgerecht verwendetes Antibiotikum" verwendet.

Acht männliche Patienten erhielten bei stationärer Aufnahme keine resistenzgerechte Antibiotikatherapie. Diese wurde dann im Verlauf an den Erreger angepasst. Somit hatten $84 \%$ der männlichen Patienten direkt bei Aufnahme eine resistenzgerechte Antibiotikagabe erhalten.

Das häufigste verwendete Antibiotikum war Ciprofloxacin (52\%).

Bei den 50 Frauen haben zu Beginn der Antibiotikatherapie 10 Patienten keine resistenzgerechte Antibiotikagabe erhalten, die im Verlauf des Krankenhausaufenthaltes jedoch angepasst wurde. Somit haben $80 \%$ der Patientinnen direkt bei Aufnahme ein resistenzgerechtes Antibiotikum erhalten.

Die beiden am häufigsten verwendeten Antibiotika der weiblichen Patienten waren Ciprofloxacin (26\%) und Cefuroxim (26\%).

Die Resistenzlage in Niedersachsen wird vom Niedersächsischen Gesundheitsamt (NLGA) jährlich erfasst. Im stationären Bereich lag die Resistenzrate 2017 bei dem am häufigsten für Harnwegsinfektionen relevanten Keim E. coli bei 21,8 \% im Hinblick auf die Wirksamkeit von Ciprofloxacin. Bei allen Keimen, die in der Harnröhre nachgewiesen werden konnten, lag die Resistenzrate für Ciprofloxacin ambulant sowie stationär $>60 \%$. Auch Cefuroxim wies 2017 eine hohe Resistenzrate bei $E$. coli von 46,6\% auf. Die geringsten Resistenzraten waren bei Fosfomycin sowie Nitrofurantoin zu finden (Niedersächsisches Landesgesundheitsamt 2017).

\subsubsection{Betrachtung des Charlson Comorbidity Index}

Um den Charlson Comorbidity Index übersichtlicher zu betrachten, wurde dieser in 2 Kategorien eingeteilt (niedrig (0 und 1 Punkt) sowie hoch (2-8 Punkte)).

Bei den Männern wiesen somit 30 Patienten einen hohen Charlson Comorbidity Index auf, von denen 5 Patienten einen Katheter bei Aufnahme bekommen hatten. Die restlichen 25 Patienten hatten keine Katheteranlage erhalten. Das mittlere Alter der Patienten mit hohem Charlson Comorbidity Index betrug 67,8 Jahre. 
20 Patienten wiesen einen niedrigen Charlson Comorbidity Index auf. Davon hatten 11 Patienten einen Katheter und 9 Patienten keine Katheteranlage erhalten. Im Mittel waren die Patienten mit niedrigem Index 45,7 Jahre alt.

12 Patientinnen wiesen einen hohen Charlson Comorbidity Index von $\geq 2$ auf, von denen 10 Patientinnen einen Katheter und lediglich 2 keinen Katheter bei Aufnahme bekommen hatten. Von den restlichen 38 Patientinnen mit niedrigem Charlson Comorbidity Index erhielten 25 Patientinnen einen Katheter und 13 keinen Katheter.

Bei der statistischen Auswertung der Frauen konnte gezeigt werden, dass ein um eine Komorbidität höhere Charlson Comorbidity Index mit einem statistisch signifikant verlängerten Krankenhausaufenthalt um 0,41 Tage einher geht. 


\section{Diskussion}

Im klinischen Alltag sind komplizierte Harnwegsinfektionen ein häufig auftretendes Krankheitsbild, dessen derzeitiges Therapieschema einer genaueren Betrachtung bedarf. Deshalb wurden im Rahmen dieser unizentrischen klinischen Studie die Krankheitsbilder Prostatitis und Epididymitis bei stationären männlichen Patienten, sowie die Pyelonephritis bei stationären weiblichen Patienten, bezüglich einer Katheterintervention untersucht.

Aktuell werden die Patienten nach EAU-Leitlinie lediglich durch eine symptomatische Therapie in Form von Analgesie sowie durch eine entsprechende Antibiotikagabe behandelt. Bei Vorstellung der Patienten entscheidet der zuständige Arzt, ob die Anlage eines Katheters einen Vorteil für den Patienten darstellt. Ein einheitliches Vorgehen für die Indikation einer Katheteranlage ist nicht beschrieben.

Für die Patienten bedeutet diese Erkrankung neben einer körperlichen Einschränkung außerdem zum Teil eine enorme Einschränkung der Lebensqualität.

Deshalb ist es für die Patienten und Ärzte von großem Interesse, ein optimales und einheitliches Therapieregime anzustreben.

In diesem Kapitel wird detailliert sowohl auf den Nutzen als auch auf die Problematik der prospektiven sowie retrospektiven Studie eingegangen und anschlieBend eine Beurteilung der erhobenen Ergebnisse durchgeführt.

\subsection{Prospektive Studie}

\subsubsection{Studiendesign}

Vor Beginn jeder Studie muss ein ausführliches Studiendesign ausgearbeitet werden, da dieses die Grundlage für die anschließende Auswertung bildet und letztendlich die Aussagekraft der Studie definiert (Röhrig et al. 2009). Altman et al. haben 1983 für die Studien medizinischer Forschung fünf Eckpfeiler festgelegt, die für eine gute Studie zu beachten sind. Dazu gehören neben der ausführlichen Planung die Durchführung, Dokumentation, Analyse und letztendlich die Publikation der Studie (Altman et al. 1983). 
Für die Planung einer Studie sollte ausreichend Zeit eingeplant werden, damit alle wichtigen Punkte einer Studie genau definiert werden und Fehler vermieden werden können. Nur so kann ein aussagekräftiges Ergebnis einer Studie erreicht werden (Röhrig et al. 2009). Wie Röhring et al. 2009 vorgeschlagen haben, werden in diesem Abschnitt die Punkte Fragestellung, Studienpopulation, Studientyp und Messverfahren in Bezug auf die prospektive Studie betrachtet.

Von essenzieller Bedeutung für die Studie ist die Fragestellung, die laut Röhring et al. 2009 genauestens die Zielgruppe definieren sollte. Die Zielgruppe für die klinische Studie wurde vor Beginn genau definiert. Es sollten nur Patienten mit Prostatitis, Epididymitis und Pyelonephritis eingeschlossen werden, die sich in urologischer stationärer Behandlung der UMG befanden. Es wurden genaue Ein- und Ausschlusskriterien definiert, sodass das zu untersuchende Patientenkollektiv eindeutig festgelegt war.

Die Studienpopulation muss eine geeignete Stichprobengröße aufweisen, damit von dem Probekollektiv auf die Grundgesamtheit geschlossen werden kann (Röhrig et al. 2009). Für diese klinische Studie wurde mithilfe eines Probekollektives die Anzahl der Studienteilnehmer ermittelt. Trotz dieser Hochrechnung erwies sich das Kollektiv für den festgelegten Zeitraum von einem Jahr als zu groß, weshalb keine 100 Patienten in die Studie eingeschlossen werden konnten. Es hatten sich unerwartet deutlich weniger Patienten in der urologischen Klinik vorgestellt als in den Vorjahren.

Man hätte rückblickend für den Einschluss von 100 Patienten entweder einen längeren Studienzeitraum wählen oder aber die Durchführung einer multizentrischen Studie in Erwägung ziehen müssen.

Der Studientyp der prospektiven Studie wurde bewusst gewählt, um die Anwendung einer Katheterintervention durch primäre Daten zu untersuchen und durch festgesetzte Studieneckpunkte (z. B. Urinstatus am dritten Tag) aussagekräftige Ergebnisse zu erzielen.

Röhrig et al. 2009 weisen ebenfalls auf geeignete Messinstrumente hin, die für eine gut durchgeführte Studie von essenzieller Bedeutung sind. Die für die klinische prospektive Studie ausgewählten klassischen Messverfahren (Laborparameter, Temperaturmessung, Urinstatus) sowie die Messverfahrung zur Ermittlung der 
Lebensqualität (SF-12-Fragebogen und VAS) zeigen die gut durchdachte Planung des Studiendesigns.

Somit kann festgehalten werden, dass die Studie zunächst gut geplant wurde und wichtige Inhalte eines guten Studiendesigns enthalten hat. Die geringe Studienteilnehmerzahl hätte durch eine multizentrische Studie verhindert werden können. Allerdings konnten einige Patienten auch aufgrund von Hindernissen in der Studiendurchführung nicht in die Studie eingeschlossen werden (siehe Kapitel 5.1.2).

\subsubsection{Studiendurchführung}

Die Durchführung bzw. der Ablauf der Studie sollte durch die erstellten Ablaufschemata für Männer und Frauen gewährleistet werden. Durch diese sollte den Ärzten durch eindeutig definierte Aussagen die Durchführung erleichtert und ein standardisiertes Vorgehen erreicht werden.

Da sich die Patienten mit komplizierten Harnwegsinfektionen erfahrungsgemäß häufig nachts, das heißt bei einem diensthabenden Arzt, vorstellen, wurde häufig aufgrund von hoher Arbeitsbelastung verpasst, die Patienten über die Studie zu informieren und einzuschließen.

Häufiger wurde direkt ein Katheter bei Aufnahme gelegt, was eine spätere Randomisierung sowie Aufnahme der Patienten in die Studie unmöglich machte.

Im Nachhinein kann man deshalb sagen, dass es von Vorteil für die prospektive Studie gewesen wäre, wenn bei entsprechenden Krankheitsbildern kein Katheter bis zum nächsten Morgen bis zur Frühbesprechung der Urologen gelegt worden wäre. Somit wäre ein Ausscheiden aus der Studie durch einen bereits bei Aufnahme gelegten Katheter nicht möglich gewesen. 


\subsection{Retrospektive Studie}

In nachfolgenden Kapiteln werden detailliert die im vierten Kapitel beschriebenen Ergebnisse sowie die Eigenschaften und Probleme der Studie diskutiert.

\subsubsection{Diskussion der Ergebnisse Männer}

\subsubsection{Katheterintervention}

Etienne et al. untersuchten 2008 aus einem Patientenkollektiv von 371 Patienten mit akuter Prostatitis, ob es einen Unterschied in der therapeutischen Vorgehensweise verschiedener Krankenhausabteilungen bei akuter Prostatitis gibt. Dafür wurden retrospektive Daten aus zwei französischen Krankenhäusern verglichen und ausgewertet. Die Studie konnte zeigen, dass es je nach Krankenhaus unterschiedliche Therapieregime bei Prostatitis gibt, wobei sich diese Studie nur auf die verwendete Antibiotikatherapie bezieht. Die Studie weist auch auf die unterschiedlichen Kriterien zur Diagnosestellung einer Prostatitis hin, die je nach Krankenhaus und vorstelligem Patientenkollektiv variieren. Allgemein kommt diese Studie zu dem Schluss, dass zwar ein ähnliches Therapieregime angewendet wird, die genaue Therapie aber je nach Krankenhaus variiert. Die Katheterintervention wurde in dieser Studie nicht beobachtet (Etienne et al. 2008).

Des Weiteren konnten keine vergleichbaren Studien zur Untersuchung der Katheterintervention bei komplizierten Harnwegsinfektionen gefunden werden.

Bei dieser retrospektiven Studie ergab sich bei den Männern, dass Patienten mit einem Katheter einen signifikant längeren Krankenhausaufenthalt von 1,36 Tagen im Vergleich zu Männern ohne Katheteranlage aufweisen.

Das könnte zum Beispiel daran liegen, dass es durch die Katheteranlage häufiger zu Infektionen kommt (Saint and Chenoweth 2003; Warren 2001) oder zum Beispiel nach Zug des Katheters Irritationen bei der Blasenentleerung entstehen (Sökeland et al. 2000), die einen verlängerten Krankenhausaufenthalt mit sich bringen. Außerdem ist ein transurethraler Katheter in der Literatur bei Prostatitis oder Epididymitis eher nicht empfohlen, was zur Verzerrung der Ergebnisse geführt haben könnte (Sökeland et al. 2000).

Am kürzesten waren Patienten im Krankenhaus, die keinen Katheter bei Aufnahme erhalten hatten (4,06 Tage). Allerdings sollte man bei diesen Patienten 
genauer die klinischen Parameter (Temperatur bei Aufnahme, Fieber, CRP- sowie Leukozytenwerte) betrachten. Diese klinischen Parameter sind in Tabelle 4 ausführlich dargestellt.

Die klinischen Parameter der männlichen Patienten ohne Katheter zeigen, dass diese im Vergleich zu Patienten, die während ihrer Erkrankung einen Katheter erhalten hatten, tatsächlich "gesünder" erscheinen. Die Patienten ohne Katheterintervention weisen eine niedrigere Körpertemperatur bei Aufnahme, ein niedrigeres CRP und seltener Fieber auf als Patienten, die einen Katheter bekommen hatten. Ausschließlich der Leukozytenwert im Mittel war geringfügig höher.

Allerdings ergibt sich lediglich für das CRP bei Aufnahme eine statistische Signifikanz von $p=0,024$.

Außerdem hatten Patienten mit erhöhtem Charlson Comorbidity Index häufiger einen Katheter bekommen als Patienten mit einem niedrigeren Index. Dieses unterstützt ebenfalls die Beobachtungen, dass die Katheteranlage mit dem Gesundheitszustand der Patienten bei Aufnahme einhergeht.

Aufgrund der retrospektiven Analyse der Patienten liegt hier wahrscheinlich ein Selektionsbias des Patientenkollektivs vor. Patienten, die bei stationärer Aufnahme geringere klinische Symptome bzw. Laborparameter aufwiesen, hatten häufiger keinen Katheter bei Aufnahme erhalten als Patienten, die schwerere körperliche Symptome oder höhere Entzündungsparameter im Labor aufwiesen. Um diesen Selektionsbias zu umgehen, ist eine prospektive Studie inklusive Randomisierung unumgänglich.

Man muss außerdem bedenken, dass bei einem kleinen Patientenkollektiv nicht alle Parameter, für die ein geringer Unterschied besteht, mit in die Studie einbezogen werden konnten und somit nicht für alle Variablen adjustiert wurde.

Außerdem wurde lediglich von den zwei Confoundern Fieber bei Krankenhausaufenthalt und Alter ausgegangen. Es wäre möglich, dass weitere Confounder vorhanden waren, möglicherweise auch von Parametern die in der klinischen Studie nicht erfasst wurden. 


\subsubsection{Alter}

Das Alter der Patienten spielt für die Krankenhausaufenthaltsdauer eine wichtige Rolle. Durch gleichzeitig steigende Komorbiditäten geht ein erhöhtes Alter häufig mit einem verlängerten Krankenhausaufenthalt einher.

Diesen Effekt zeigten Librero et al. 1999 mit der Untersuchung des Einflusses des Charlson Comorbidity Index auf die stationäre Verweildauer. Die Studie konnte zeigen, dass ein erhöhter Index mit einem verlängerten Krankenhausaufenthalt einhergeht (Librero et al. 1999).

Somit würde man davon ausgehen, dass ältere Patienten, die häufiger Komorbiditäten haben, in der Regel einen längeren stationären Krankenhausaufenthalt aufweisen.

Interessanterweise konnte in dieser Studie gezeigt werden, dass Männer mit Prostatitis oder Epididymitis $\leq 63$ Jahre, die bei Aufnahme einen Katheter erhalten hatten (unabhängig davon, ob SPK oder DK), einen verlängerten Krankenhausaufenthalt von 2,0 Tagen aufweisen, im Vergleich zu gleichaltrigen Patienten ohne Katheter.

Schaut man sich die klinischen Parameter der Subgruppen an erkennt man, dass das jüngere männliche Patientenkollektiv im Vergleich nicht wesentlich gesünder oder kränker erscheint. Das jüngere Patientenkollektiv hatte im Mittel zwar einen CRP-Wert bei Aufnahme von 76,84 mg/dl, wohingegen die Männer > 63 Jahre einen Wert von 112,53 mg/dl aufwiesen, allerdings zeigten sich im Hinblick auf Temperatur und Leukozytenwerte bei Aufnahme keine wesentlichen Unterschiede des Gesundheitszustandes.

Wenn man weiterhin davon ausgeht, dass ein erhöhtes Alter den Krankenhausaufenthalt verlängert und die Patienten klinisch nicht kränker anzusehen sind, kann der Effekt des verlängerten Krankenhausaufenthaltes bei den jüngeren Patienten möglicherweise direkt auf die Katheteranlage und deren potenzielle Komplikationen zurückzuführen sein.

Eine Katheteranlage birgt immer das Risiko für Verletzungen, subjektive Beeinträchtigung der Patienten, Komplikationen nach Entfernung des Katheters und am häufigsten für Infektionen (Sökeland et al. 2000). Einige Bakterien (z. B. Staphylococcus aureus (S. aureus)) haben im Laufe der Zeit gelernt, einen Biofilm zu 
bilden. Sie schließen sich wie ein Netzwerk zusammen, sind somit widerstandsfähiger und schlechter durch Antibiotika behandelbar (Saint und Chenoweth 2003). Biofilme bilden sich häufig im Krankenhaus an medizinischen Instrumenten und Geräten. Katheterdrainagen stellen somit einen Risikofaktor für Biofilme und somit für Infektionen dar. Harnwegsinfektionen sind mit die häufigsten nosokomialen Infektionen (Esposito et al. 2008).

Patienten > 63 Jahre hatten häufiger einen transurethralen Katheter bekommen, wohingegen vor allem Patienten $\leq 63$ Jahre einen SPK bekommen hatten.

Somit wäre es möglich, dass die verlängerte Krankenhausaufentshaltsdauer bei Patienten mit Katheterdrainage mit den oben beschriebenen Komplikationen zusammenhängt.

Bei den älteren Männern > 63 Jahre konnte kein statistischer Zusammenhang zwischen der Katheteranlage und dem Krankenhausaufenthalt festgestellt werden. Möglicherweise liegt das an dem kleinen Patientenkollektiv und der damit einhergehenden zu geringen Power.

\subsubsection{Antibiotikatherapie}

Die Antibiotikatherapie stellt bei den komplizierten Harnwegsinfektionen den momentan wichtigsten therapeutischen Stellenwert dar (EAU-Guidelines Prostatitis/Epididymitis 2015, Gujadhur und Aning 2015).

Bei der Subgruppenanalyse der Männer > 63 Jahre wurde mittels statistischer Berechnungen erfasst, dass dieses Patientenkollektiv bei resistenzgerechter Antibiotikagabe einen verkürzten Krankenhausaufenthalt von 5,06 Tagen aufweist. Diese Beobachtung stimmt mit dem allgemeinen medizinischen Verständnis überein, dass nur eine resistenzgerechte Antibiotikatherapie die Erreger bekämpft und den Zustand des Patienten dadurch schneller verbessert. 


\subsubsection{Diskussion der Ergebnisse Frauen}

\subsubsection{Katheterintervention}

Für das Therapieregime der akuten Pyelonephritis, bezogen auf die Anwendung eines Katheters, konnten keine vergleichbaren Studien gefunden werden. Das Hauptaugenmerk bisheriger Studien richtete sich auf die korrekte Antibiotikatherapie und das Erreger- sowie Resistenzspektrum (Etienne et al. 2014; Efstathiou et al. 2003).

Bei dem Patientenkollektiv dieser Studie konnte kein statistischer Zusammenhang zwischen der Katheteranlage und der Krankenhausaufenthalsdauer festgestellt werden. Das kann auf der einen Seite daran liegen, dass das Patientenkollektiv der 50 Frauen zu gering ist oder auf der anderen Seite damit zusammenhängen, dass die Katheteranlage tatsächlich keinen Einfluss auf die Länge des Krankenhausaufenthaltes hat. Um diese Aussage definitiv treffen zu können, muss diese Form der Studie an einem größeren Kollektiv, am besten in Form einer multizentrischen Studie, untersucht werden

Obwohl es keinen statistisch signifikanten Zusammenhang zwischen dem Krankenhausaufenthalt und der Katheteranlage in diesen Berechnungen gab, erkennt man in den absoluten Zahlen einen Trend.

Im Mittel blieben Frauen, die bei Aufnahme einen Katheter bekommen hatten, 6 Tage im Krankenhaus, wohingegen Frauen die keinen Katheter erhalten hatten, 5 Tage im Krankenhaus blieben. Allerdings muss hier ebenfalls genauer betrachtet werden, ob der Effekt wirklich auf die Katheterlage zurückzuführen ist oder ob die Patienten ohne Katheter nicht so schwer von dem Krankheitsbild betroffen waren. Dafür ist es wichtig, sich die klinischen Parameter der Patienten erneut anzuschauen.

Die klinischen Parameter der Patientinnen mit und ohne Katheter finden sich in Tabelle 10.

Hier kann man erkennen, dass die Patientinnen ohne Katheter in allen klinischen Parametern niedrigere Werte aufweisen als die Patientinnen, die einen Katheter erhalten hatten. Somit könnte man annehmen, dass die Patientinnen ohne Katheter aufgrund ihres allgemein besseren Gesundheitszustandes einen kürzeren Krankenhausaufenthalt aufwiesen als Patientinnen, die einen Katheter gelegt 
bekommen hatten. Um einen Selektionsbias auszuschließen, muss dieser Effekt ebenfalls in einer prospektiven Studie untersucht werden.

Außerdem lässt sich mit diesen Erkenntnissen nicht die Frage beantworten, ob eine Katheteranlage zu einer schnelleren Genesung der Patienten führt. Dafür müsste durch Randomisierung der Patientinnen, wie ursprünglich geplant, die Katheteranlage zugeteilt werden, um den Effekt, dass nicht so schwer betroffene Patienten keinen Katheter erhalten und somit durch ihren klinischen Zustand einen kürzen Krankenhauaufenthalt aufweisen, auszuschließen. Die Randomisierung würde ebenfalls einen möglichen Selektionsbias ausschließen.

\subsubsection{Alter}

Muneishi et al. untersuchten 2016 an 106 Patientinnen mit Pyelonephritis, die in einem japanischen Krankenhaus zwischen 2007 und 2012 stationär behandelt wurden, welche Risikofaktoren zu einem verlängerten Krankenhausaufenthalt führen. Sie konnten zeigen, dass Frauen mit septischem Schock, malignen urologischen Erkrankungen, einem Alter über 65 Jahre oder neurogenen Blasenstörungen einen verlängerten Krankenhausaufenthalt aufweisen. Somit stellt in diesem Patientenkollektiv das Alter > 65 Jahre einen Risikofaktor für einen verlängerten Krankenhausaufenthalt für Patienten mit Pyelonephritis dar (Muneishi et al. 2016).

Efstathiou et al. untersuchten 2003 retrospektiv an 225 Patienten mit Pyelonephritis (102 Männer und 120 Frauen) die Risikofaktoren für einen verlängerten stationären Krankenhausaufenthalt. Sie konnten mit dieser Studie ebenfalls zeigen, dass bei Frauen $>65$ Jahre mit Diabetes mellitus oder einer langen Katheterlage während des Krankenhausaufenthalts mit einem verlängerten Aufenthalt gerechnet werden sollte, wenn es initial zu einer nicht optimalen Antibiotikatherapie gekommen war (Efstathiou et al. 2003).

In dieser klinischen retrospektiven Studie konnte bei Frauen mit Pyelonephritis $(n=50)$ kein Zusammenhang zwischen dem Alter und dem Krankenhausaufenthalt festgestellt werden. Möglicherweise liegt das an der zu geringen Studienteilnehmerzahl, durch die ein potenzieller statistischer Zusammenhang nicht hergestellt werden konnte. Auf der anderen Seite muss man erwähnen, dass in dieser Studie 
das mittlere Patientenalter 35 Jahre betrug (SD 19 Jahren), und lediglich 7 Frauen $>65$ Jahren mit in die Studie eingeschlossen werden konnten. In der Studie von Muneishi et al. waren 69 von 106 Patienten älter als 65 Jahre. Somit kann ein potenzieller Effekt des Alters in Bezug auf die Krankenhausaufenthaltsdauer aufgrund eines jüngeren Patientenkollektivs vermutlich nicht nachgewiesen werden.

\subsubsection{Antibiotikagabe}

Die Leitlinien zur Antibiotikatherapie komplizierter Harnwegsinfektionen sind aufgrund der Resistenzentwicklung der Erreger einem ständigen Wandel unterworfen.

Etienne et al. untersuchten 2014 in einer Studie die Resistenzraten von E. coli bei 156 Pyelonephritispatientinnen. Es konnte gezeigt werden, dass $82 \%$ der Pyelonephritiden von E. coli ausgelöst wurden. In der Therapie zeigten sich Resistenzraten von $15 \%$ bei den Fluorchinolonen sowie $6 \%$ bei den Cephalosporinen der dritten Generation. Außerdem konnte gezeigt werden, dass das Risiko für Resistenzen mit den Risikofaktoren der Patienten ansteigt (Etienne et al. 2014).

Laut der neuen S3-Leitlinie 2017 (AWMF-Leitlinie unkomplizierte Harnwegsinfektionen 2017) hängt die Therapie der Pyelonephritispatienten weiterhin entscheidend von der Resistenzrate der Erreger ab. Das bisher häufig bei Pyelonephritis verwendete Fluorchinolon Ciprofloxacin ist aufgrund der hohen Resistenzrate in Niedersachen bei E. coli von aktuell 15,3\% nicht mehr Mittel der ersten Wahl. Stattdessen sollte auf andere Antibiotika wie Cephalosporine der dritten Generation (Cefpodoxim) mit einer momentanen Resistenzrate in Niedersachsen von $8,7 \%$ bei $E$. coli zurückgegriffen werden. Das in der retrospektiven Studie ebenfalls häufig verwendete Antibiotikum Cefuroxim sollte aufgrund aktuell hoher Resistenzraten in Niedersachen von $43,9 \%$ bei E. coli ebenfalls nicht mehr zur Therapie der Pyelonephritis verwendet werden (AWMF-Leitlinie unkomplizierte Harnwegsinfektionen 2017).

Alternativ kommen aktuell Aminoglykoside oder Carbapeneme zum Einsatz, die jedoch ausschließlich intravenös verabreicht werden können. Gegebenfalls muss nach Erhalt des Antibiogramms das Antibiotikum resistenzgerecht angepasst werden. 
In dieser retrospektiven Analyse wurden am häufigsten die beiden Antibiotika Cefuroxim und Ciprofloxacin verwendet, um die Pyelonephritis zu therapieren. Ciprofloxacin und Cefuroxim stellen aktuell zwar keine leitliniengerechte Therapie mehr dar, sind aber aufgrund der retrospektiven Datenerhebung bis 2013 Mittel der ersten Wahl bei Pyelonephritis gewesen. Deshalb ist es von enormer Bedeutung, das Antibiotikaregime stetig zu überprüfen und an die regionalen Gegebenheiten anzupassen.

\subsubsection{Komorbiditäten}

Wie in Kapitel 5.2.2.2 beschrieben, konnte die Studie von Efstathiou et al. 2013 einige Komorbiditäten als Risikofaktoren für einen verlängerten Krankenhausaufenthalt bei Patientinnen mit Pyelonephritis feststellen.

Librero et al. untersuchten 1999 an Daten aus 106,67 Krankenhausaufenthalten aus Valencia (Spanien) unabhängig von dem Krankheitsbild einen möglichen Zusammenhang zwischen der Krankenhausaufenthaltsdauer und dem Charlson Comorbidity Index. Es konnte festgestellt werden, dass die Krankenhausaufenthaltsdauer bei erhöhtem Charlson Comorbidity Index generell ansteigt. Außerdem steigt mit erhöhtem Index die Krankenhausmortalität (Librero et al. 1999).

Bei der statistischen Auswertung dieser klinischen Studie der Frauen konnte ebenfalls gezeigt werden, dass ein um eine Komorbidität höherer Charlson Comorbidity Index mit einem statistisch signifikant verlängerten Krankenhausaufenthalt für weibliche Patienten um 0,41 Tage einhergeht. Somit zeigt diese retrospektive Studie ähnliche Ergebnisse wie die beiden großen Studien von Efstathiou und Librero. 


\subsection{Stärken der Studie}

Die Planung sowie der Ablauf der prospektiven Studie wurden ausführlich durchdacht und durch ein genaues Studiendesign beschrieben. Der Gedanke, die Patienten inklusive Follow-Up zu untersuchen und zu erfassen, zeigt die weitreichenden Überlegungen. Patientenaufklärung und Einverständniserklärung wurden extra für die Studie erstellt und befinden sich im Anhang.

Die erstellten Patientenmappen, die jeder Patient bei Aufnahme erhalten sollte und die neben der Aufklärung auch die VAS und den SF-12-Fragebogen enthielten, sollten die Patienten von der Studie überzeugen und den Ärzten als Hilfe für die Aufklärung über die Studie dienen. In einer größer angelegten Studie stellt diese Art Hilfsmittel sicherlich für Patient und Arzt eine Erleichterung dar.

Der Studiengedanke, also die Untersuchung des Therapieregimes bei komplizierten Harnwegsinfektionen in der Urologie, ist sehr innovativ und für den klinischen Altag enorm wichtig. Es konnten keine vergleichbaren Studien gefunden werden, in denen die Anwendung eines Katheters, insbesondere des SPK, bei komplizierten Harnwegsinfektionen untersucht wurde.

\subsection{Schwächen der Studie}

\subsubsection{Patientenkollektiv}

Wie bereits mehrfach beschrieben, stellt das kleine Patientenkollektiv den größten Kritikpunkt der retrospektiven Studie dar. In die retrospektive Studie wurden 50 männliche und 50 weibliche Patienten der Jahre 2013 - 2017 eingeschlossen. Da die Daten der Urologie seit 2015 digitalisiert erfasst werden, ließen sich diese Datensätze gut und fast vollständig erheben. Je weiter man allerdings im Aktenarchiv der UMG in den Jahren zurückgeht, desto schwieriger war es, die geforderten Patientendaten vollständig zu erheben. Aufgrund dieser lückenhaften Dokumentation der vergangenen Jahre flossen lediglich die ursprünglich geplanten 50 Patientendaten in die Berechnungen der Studie ein. 


\subsubsection{Zielvariable}

Die ursprünglich geplante Fragestellung, der Anlage eines ausschließlich suprapubischen Katheters, konnte aufgrund des zu kleinen Patientenkollektivs nicht statistisch ausgewertet werden. Zur statistischen Auswertung wurde lediglich die Zielvariable zusammenfassend als Katheteranlage (SPK und DK) betrachtet.

Schaut man sich die Verteilung der Katheter bei den 50 männlichen Patienten an, erkennt man, dass 27 Patienten einen transurethralen Katheter und 7 Patienten einen SPK bei Aufnahme erhalten hatten.

Die Patienten die einen transurethralen Katheter erhalten hatten, waren im Vergleich zu den Patienten die einen SPK bei Aufnahme bekommen hatten länger im Krankenhaus (SPK 4,86 vs. DK 6,70 Tage)

Das könnte daran liegen, dass die Ableitung bei komplizierten Harnwegsinfektionen über einen transurethralen Katheter zu einer Verschleppung der Keime führt und eine zusätzliche Reizung der betroffenen Stelle darstellt (Sökeland et al. 2000; Manski 2017).

Die ursprünglich geplante 1:1-Randomisierung sollte dafür sorgen, dass zwei gleichgroße Patientenkollektive mit jeweils 25 Patienten mit und ohne Katheter entstehen, um eine optimale Vergleichbarkeit der Versuchsgruppen gewährleisten zu können. Aufgrund der ausschließlich retrospektiv durchgeführten Studie ist die Vergleichbarkeit der einzelnen Kathetergruppen aufgrund einer unterschiedlichen Gruppengröße somit nicht gewährleistet.

Eine abschließende Aussage bezüglich der Anlage einer suprapubischen Harnableitung kann somit nicht getroffen werden.

\subsubsection{Zeitliche Erfassung der SPK-Liegedauer}

Während der Erfassung der Patientendaten stellte sich heraus, dass die Katheterliegedauer der Patienten mit SPK-Anlage lediglich bis zum Zeitpunkt der Entlassung berechnet werden konnte. Die Patienten werden in den meisten Fällen mit liegendem SPK aus dem Krankenhaus entlassen und werden gebeten, sich zur Nachsorge beim niedergelassenen Urologen vorzustellen, der dann den suprapubischen Katheter entfernt. Somit gibt es keinen Nachweis über die korrekte Liegedauer des SPK. Hierbei wäre es für eine nächste Studie notwendig, die 
ambulant behandelnden Urologen mit einzubeziehen, um ein korrektes und genaues Ergebnis der Liegezeit zu erhalten. Allerdings gestaltet sich dieses wahrscheinlich aufgrund des Klinikalltags und der stetig steigenden Arbeitsbelastung der Ärzte als schwierig umsetzbar. Eine andere Möglichkeit wäre, die Patienten wieder in die Klinik einzubestellen, um dort den Katheter zu entfernen und den genauen Zeitpunkt zu dokumentieren. Dieses würde allerdings auch eine Mehrbelastung für die Klinikärzte bedeuten. Außerdem stellt sich die Frage, ob die Patienten diesen Termin einhalten oder z. B. das Krankenhaus / den behandelnden Arzt wechseln und somit wichtige Studiendaten verloren gehen. 


\subsection{Ausblick /Empfehlungen}

Die Ergebnisse der retrospektiven Studie gilt es in einer größer angelegten klinischen prospektiven Studie zu überprüfen. Um ein ausreichend großes Patientenkollektiv für statistische Berechnungen zu erhalten, würde eine multizentrisch angelegte Studie einen großen Vorteil darstellen. Außerdem kann durch die prospektive Studie, wie ursprünglich geplant, durch 1:1-Randomisierung sichergestellt werden, dass kein Selektionsbias vorhanden ist. Gegebenenfalls könnte man auch mithilfe einer dritten Patientengruppe die Anwendung des transurethralen Dauerkatheters mituntersuchen.

Eines der größeren Probleme dieser Studie war es, dass die Patienten nicht bei Aufnahme sofort auf die Studie hingewiesen und nach Prüfung aller Kontraindikationen sofort eingeschlossen wurden. Durch klar definierte Abläufe des ärztlichen Personals, z. B., dass jeder Patient mit einer komplizierten Harnwegsinfektionen erst nach der Vorstellung in der Frühbesprechung am nächsten Morgen einen Katheter zugeteilt bekommt, kann gewährleistet werden, dass der Patient nicht durch eine vorzeitige Katheteranlage aus der Studie ausscheidet. Indikationen für eine sofortige Katheteranlage müssen natürlich berücksichtigt werden.

Außerdem sollte, je nach Größe und Auslastung des Krankenhauses, darüber nachgedacht werden, ambulante Urologen mit in die Studie einzubeziehen, um ein vollständiges Follow-Up der Patienten zu gewährleisten. 


\subsection{Abschließende Beurteilung}

Obwohl es in der Durchführung dieser geplanten prospektiven Studie zu einigen unerwarteten Problemen gekommen ist, zeigt die Betrachtung der retrospektiven Daten, dass der Gedanke hinter der Studie, nämlich den Stellenwert der Katheteranlage bei komplizierten Harnwegsinfektionen zu untersuchten, ein wichtiger Ansatz zur Optimierung des Therapieregimes von komplizierten Harnwegsinfektionen darstellt. Für betroffene Patienten bedeutet eine komplizierte Harnwegsinfektion eine Einschränkung der Lebensqualität, die es durch eine optimale Behandlung zu beseitigen gilt.

Es konnte keine vergleichbare Studie zu dem Thema der Katheterintervention bei Prostatitis/Epididymitis gefunden werden, was zeigt, dass ein wichtiger innovativer Gedanke hinter der Studie steckt und weshalb eine erneut angelegte prospektive Studie zu diesem Thema wichtige Erkenntnisse für diese Krankheitsbilder bringen kann.

Deshalb wird der Ansatz dieser Studie als extrem wichtig eingeschätzt. 


\section{Zusammenfassung}

Komplizierte Harnwegsinfektionen wie Prostatitis, Epididymitis sowie Pyelonephritis sind wichtige und häufige Krankheitsbilder in der Urologie. Für betroffene Patienten steht neben den körperlichen Beschwerden und Einschränkungen vor allem die eingeschränkte Lebensqualität im Vordergrund. Aktuell werden die Patienten leitliniengerecht mittels einer symptomatischen Therapie, bestehend aus Bettruhe, Analgesie und Flüssigkeitsgabe sowie einer kalkulierten Antibiotikatherapie, behandelt. Die Anlage einer Harnableitung wird individuell je nach Gesundheitszustand des Patienten entschieden. Ein einheitliches Konzept zur Indikation einer Katheteranlage besteht derzeit nicht. Um den Patienten eine optimale Versorgung zu gewährleisten und somit ein optimales Therapieregime für die Patienten zu entwickeln, wurde diese klinische Studie konzipiert. Die klinische prospektive Studie wurde für ein Jahr geplant und vollzogen, lieferte aber aufgrund eines zu geringen Patienteneinschlusses kein Ergebnis. Daraufhin wurde die prospektive Studie durch eine retrospektive Betrachtung des Patientenkollektivs der Universitätsmedizin Göttingen aus den Jahren 2013 - 2017 ersetzt. Die aufgestellte Hypothese war, dass die Anlage eines Katheters im Vergleich zu einem Verzicht auf die Katheteranlage zu keinem Unterschied in der Krankenhausaufenthalts-dauer führt. In der retrospektiven Studie wurden 50 männliche Patienten mit Prostatitis und Epididymitis sowie 50 weibliche Patienten mit Pyelonephritis untersucht, die sich in stationärer Behandlung der UMG befanden.

Um die erhobenen Daten auszuwerten, wurden die Statistikprogramme $R$ und RStudio verwendet. Die statistischen Berechnungen beruhen auf der linearen Regression und umfassen univariate sowie multivariable Analysen. Als statistischer Endpunkt wurde der Krankenhausaufenthalt in Tagen angenommen. Das Signifikanzniveau lag bei $0,05 \%$.

Die Analyse der 50 männlichen Patienten mit Prostatitis und Epididymitis zeigte, dass die Anlage eines Katheters zu einem verlängerten Krankenhausaufenthalt von 1,36 Tagen führt (95\% KI 0,06 bis 2,66). Hierbei konnte aufgrund des kleinen Patientenkollektivs nicht auf die Art des Katheters eingegangen werden.

Patienten ohne Katheter waren wesentlich kürzer im Krankenhaus als Patienten mit Harnableitung. Man muss allerdings berücksichtigen, dass bei Aufnahme ge- 
sünder erscheinende Patienten (das heißt Patienten mit niedrigeren Infektwerten, geringerem Krankheitsgefühl etc.) zunächst keinen Katheter bekommen hatten und somit eventuell ein Selektionsbias vorliegt. Allerdings wäre es auch möglich, dass es bei Patienten mit Katheter häufiger zu Komplikationen wie z. B. Infektionen kommt.

In der Subgruppenanalyse der Männer > 63 Jahre konnte kein statistischer Zusammengang zwischen einer Katheteranlage und dem Krankenhausaufenthalt festgestellt werden $(p=0,9)$. Allerdings ließ sich beim männlichen Kollektiv $\leq 63$ Jahre zeigen, dass bei diesen Patienten eine Katheteranlage zu einem um 2,0 Tage (95\% KI 1,81 bis 5,48) verlängerten Krankenhausaufenthalt führt. Mehrere Studien haben gezeigt, dass ein erhöhtes Alter der Patienten den Krankenhausaufenthalt verlängert. In dieser Studie waren die Patienten ohne Katheteranlage nicht wesentlich gesünder oder kränker als Patienten, die einen Katheter bei Aufnahme bekommen hatten. Deshalb kann davon ausgegangen werden, dass die Anlage eines Katheters in diesem Patientenkollektiv direkt im Zusammenhang mit der Krankenhausaufenthaltsdauer der Patienten steht.

In der Analyse der 50 weiblichen Patienten mit Pyelonephritis konnte kein statistischer Zusammenhang ( $p$ - Wert =0,614) zwischen der Katheteranlage und dem Krankenhausaufenthalt festgestellt werden. Möglicherweise kann dieses Phänomen auf das kleine Patientenkollektiv zurückzuführen sein. Auf der anderen Seite steht der bessere Gesundheitszustand bei Patienten ohne Katheter im Vordergrund. Dieses Ergebnis sollte in einem größeren Kollektiv erneut untersucht werden. Auch im Hinblick auf das Alter der Patientinnen in dieser Studie konnte kein Zusammenhang zwischen der Krankenhausaufenthaltsdauer und dem Alter ermittelt werden. Die am häufigsten verwendeten Antibiotika bei Pyelonephritis waren Ciprofloxacin und Cefuroxim. Diese stellen in den neueren Leitlinien zwar keine primäre Therapie mehr dar, waren aber zum Zeitpunkt der retrospektiv erfassten Patienten eine richtige Behandlungsoption.

Die ursprüngliche Fragestellung des Stellenwertes einer Katheteranlage, vor allem der suprapubischen Katheteranlage, konnte abschließend nicht eindeutig geklärt werden. Aufgrund der ausschließlich retrospektiven Betrachtung ist die Aussagekraft der Daten im Vergleich zur prospektiven Analyse eingeschränkt. Deshalb ist es notwendig, eine größer ausgelegte prospektive Studie, eventuell auch 
multizentrisch, durchzuführen, in der durch eine Randomisierung eine optimale Beurteilung der verschiedenen Katheteranlagen, wie ursprünglich geplant, möglich ist.

Es konnten keine vergleichbaren Studien zu dem Thema der Katheterintervention bei Prostatitis/Epididymitis gefunden werden, was zeigt, dass ein wichtiger innovativer Gedanke hinter der Studie steckt und weshalb eine erneut angelegte Studie zu diesem Thema wichtige Erkenntnisse für diese Krankheitsbilder bringen wird. 


\section{Anhang}

\section{Eingereichter Ethikantrag}

\section{Kurzprotokoll}

\section{Zur Einreichung bei der Ethik-Kommission}

Medizinische Fakultät der Georg-August-Universität Göttingen

Von-Siebold-Straße 3

37075 Göttingen

\section{Titel des Projektes:}

„Untersuchung des Stellenwerts von transurethraler und suprapubischer Harnableitung in der Therapie von Prostatitis, Epididymitis und Pyelonephritis“

Kurztitel:

Die Rolle der Harnableitung im therapeutischen Management komplizierter Harnwegsinfektionen

\section{Antragsteller:}

Dr. O. Hahn, PD Dr. S. Ahyai, Prof. Dr. L. Trojan, M. Stäblein

Datum der Antragstellung: 06.04.2016

\section{Antragsteller}

- Dr. med. O. Hahn Klinik für Urologie Universitätsmedizin Göttingen Robert-Koch-Str. 40, 37075 Göttingen Tel.: 0551/39-20748

Email: oliver.hahn@med.uni-goettingen.de 
- PD Dr. med. S. Ahyai

Klinik für Urologie

Universitätsmedizin Göttingen

Robert-Koch-Str. 40, 37075 Göttingen

Tel: 0551/39-22641

Email: sascha.ahyai@med.uni-goettingen.de

- Prof. Dr. med. L. Trojan

Klinik für Urologie

Universitätsmedizin Göttingen

Robert-Koch-Str. 40, 37075 Göttingen

Tel: 0551/39-66166

Email: lutz.trojan@med.uni-goettingen.de

- Marlena Schubert geb. Stäblein

Doktorandin

Tel: $x x x$

Email: marlena.schubert@stud.uni-goettingen.de

Eine Verschwiegenheitserklärung wurde mit der Doktorandenvereinbarung unterschrieben.

\section{Studienziel:}

Prostatitis, Epididymitis und Pyelonephritis sind Folge aufsteigender Infektionen aus dem Urogenitaltrakt und können zusammenfassend als komplizierte Harnwegsinfekte bezeichnet werden.

Das Therapieregime variiert hierbei zwischen abakteriellen und bakteriellen Infektionen.

Neben der vorrangigen symptomatischen Therapie, bestehend aus Bettruhe sowie antipyretischen und analgetischen Maßnahmen, steht bei den bakteriell verursachten Infektionen die antibiotische Therapie im Vordergrund [1].

Bei Harnabflussstörungen (z. B. relevanter Restharnbildung) ist die Anlage eines Katheters obligat [1]. Hierbei unterscheidet man zwischen transurethraler und suprapubischer Harnableitung [2]. Bei Prostatitis und Epididymitis verwendet man, um eine Passage der infizierten Areale zu vermeiden, vorrangig einen suprapubischen Katheter. Im Gegensatz dazu ist die transurethrale Harnableitung die erste Wahl bei Pyelonephritis [2]. Grund für die Anlage eines Katheters ist, dass eine gefüllte Harnblase bei urologischen Infektionen ein Keimreservoir darstellt, welches durch eine konstante Urinableitung beseitigt werden kann. Bei Patienten mit komplizierten Harnwegsinfekten ohne relevante Harnabflussstörung ist die Notwendigkeit einer Harnableitung umstritten, systematische Untersuchungen hierzu wurden bislang nicht durchgeführt. 
In dieser klinisch-randomisierten Studie soll überprüft werden, ob sich durch eine generelle suprapubische Harnableitung bei Männern mit Prostatitis und Epididymitis ein Benefit für die Patienten in Hinblick auf mögliche Komplikationen, Krankheitsverlauf, Genesung und Wiedereingliederung in den Arbeitsalltag ableiten lässt. Bei Frauen soll analog hierzu der Benefit einer transurethralen Katheteranlage bei Pyelonephritis untersucht werden.

Hierzu sollen 50 männliche Patienten mit Epidididymitis/Prostatitis und 50 weibliche Patientinnen mit Pyelonephritis untersucht werden. Patienten mit Harnabflussstörung erhalten hierbei in jedem Falle einen Katheter, Patienten ohne Harnabflussstörung werden in 2 Schenkel randomisiert.

Fernziel der Studie ist eine Einschätzung, ob die Anlage eines suprapubischen Katheters bei jedem Patienten mit Prostatitis und Epididymitis einen therapeutischen Vorteil für die Betroffenen darstellt oder ob auf die invasive Anlage dieser Harnableitung verzichtet werden kann.

\section{Wissenschaftlicher Hintergrund}

\subsection{Prostatitis}

Die akute Prostatitis ist eine schmerzhafte Entzündung der Prostata, die mit starken Schmerzen im Bereich von Skrotum, Penis, Harnblase oder unterem Rücken und einer Harnwegssymptomatik einhergeht [1]. Die Prävalenz der Prostatitis liegt zwischen 5-10\% [3].

Üblicherweise handelt es sich bei der Prostatitis um eine aufsteigende bakterielle Infektion aus der die Prostata durchziehenden Urethra.

Risikofaktoren neben einem Harnwegsinfekt sind Harnröhrenengen, Katheterdauerversorgung, Prostatabiopsien und MSM, bei dem es zu einer Verschleppung von Bakterien kommen kann.

Die häufigsten Erreger der Prostatitis sind gramnegative Enterobakterien wie z. B Escherichia coli und Proteus mirabilis [1] [4]. Zusätzlich müssen sexuell übertragbare Erreger in Erwägung gezogen werden.

Die akute Prostatitis geht in den meisten Fällen mit einer Harnwegssymptomatik im Sinne von Dysurie, Algurie, Pollakisurie, Harnstrahlabschwächung, Testalgien sowie Schmerzen in Damm- und Leistenbereich einher [3].

Die Diagnose lässt sich durch die Laboruntersuchung und die digital-rektale Untersuchung (DRU) erhärten. In der DRU findet sich eine druckschmerzhafte Prostata, im Blut zeigen sich eine Leukozytose sowie ein erhöhtes CRP und BSG [4].

Des Weiteren kann das Prostata-spezifische Antigen (PSA) im Blut bestimmt werden. Dieses kann den Verdacht auf eine Prostatitis unterstützen, ist jedoch wenig spezifisch.

Die Transrektale-Ultraschalluntersuchung (TRUS) kann gefährliche Komplikationen wie Prostataabszesse ausschließen, gehört aber nach EAU-Leitlinie nicht zur Standarddiagnostik [1]. 
Therapeutisch beginnt man mit einer kalkulierten Antibiotikatherapie, bei der vor allem Fluorchinolone eingesetzt werden. Diese weisen ein breites Wirkungsspektrum auf und besitzen eine gute Gewebegängigkeit für die Prostata [5]. Symptomatisch wird zudem eine Analgesie begonnen. Die Notwendigkeit einer suprapubischen Harableitung ist umstritten, eine transurethrale Ableitung ist hier kontraindiziert [1]. In ca. 5-10\% der Fälle kommt es zum Auftreten einer chronischen Prostatitis [6].

\subsection{Epididymitis}

Die Epididymitis ist definiert als akute oder chronische Entzündung des $\mathrm{Ne}$ benhodens [1] [7]. Risikofaktoren und Erregerspektrum entsprechen weitestgehend denen der akuten Prostatitis.

Klinisch zeigen sich hier plötzliche, massive Schmerzen sowie eine Schwellung und Überwärmung des Skrotums. Die Patienten haben ein ausgesprochenes Krankheitsgefühl und können hohes Fieber aufweisen.

Die Diagnose wird anhand der klinischen Untersuchung, der digitalen rektalen Untersuchung (DRU) sowie einer Urinanalyse gestellt, bei der sich massive Leukozyten nachweisen lassen.

Das therapeutische Vorgehen bei Epididymitis besteht ebenfalls in der symptomatischen und antibiotischen Therapie.

An erster Stelle haben die Patienten strikte Bettruhe, sie sollten den Hoden hochlagern und kühlen. Analgetika wie nicht-steroidale Antiphlogistika (Ibuprofen, Diclofenac) können die Schmerzen lindern.

In der antibiotischen Therapie werden ebenfalls laut den Europäischen Leitlinien die Fluorchinolone empfohlen, da sie durch ihr breites Wirkungsspektrum eine große Anzahl an Keimen erfassen und eine gute Gewebegängigkeit aufweisen [1]. Analog zur Prostatitis ist die transurethrale Harnableitung kontraindiziert, eine suprapubische Ableitung kann diskutiert werden [1]. In 15\% der Fälle geht die akute Epididymitis in eine chronische Form über [1] [7].

\subsection{Pyelonephritis}

Die Nierenbeckenentzündung gehört ebenfalls zu den komplizierten Harnwegsinfekten, die häufig aus einer Zystitis hervorgehen. Dabei sind Frauen aufgrund der anatomisch kürzeren Urethra häufiger betroffen als Männer [8].

Die Auslöser sind wie bei Prostatitis und Epididymitis eine Reihe von Darmbakterien wie E. coli, Enterokokken, Proteus mirabilis u.v.m.

Klinisch zeigen sich typischerweise Pollakisurie, Dysurie, Algurie, Flankenschmerzen und Fieber $>38$ Grad. Häufig ist die Entzündung von unspezifischen Symptomen wie Schüttelfrost, Übelkeit, Erbrechen und Tachykardie begleitet [9].

Im Labor zeigen sich erhöhte CRP- und BSG-Werte, eine Leukozytose oder Leukopenie und ggf. einen Anstieg des Kreatinins. Im Urinstatus kann eine erhöhte Aktivität der Granulozyten-Esterase sowie Nitrit-Positivität beobachtet werden. Sonographisch kann die Entzündung mit einer Ektasie des Nierenbe- 
ckenkelchsystems, einer Schwellung des Nierenparenchyms sowie im Doppler mit einer Hyperperfusion der Niere einhergehen [10].

Die allgemeine Therapie besteht aus Symptomlinderung, Bettruhe, Antiphlogistika und Flüssigeitsgabe.

Zusätzlich muss je nach Keimspektrum eine kalkulierte Antibiose begonnen werden. Primär werden Fluorchinolone oder Cephalosporine der Drittgeneration eingesetzt [10].

Aufgrund des erhöhten Risikos einer Urosepsis durch vesikoureteralen Reflux sollte primär die Einlage eines transurethralen Katheters erfolgen [8].

\subsection{Die Harnableitung}

Bei der kontinuierlichen Harnableitung kann zwischen zwei Formen unterschieden werden: dem transurethralen und dem suprapubischen Katheter.

\subsubsection{Der transurethrale Blasenkatheter}

Der transurethrale Katheter ist die erste Wahl bei der Harnableitung und kann in vielen klinischen Situationen gelegt werden. Die Indikationen für solch einen Katheter bestehen z. B. in der Bilanzierung der Urinmenge, bei bettlägerigen Patienten z. B. nach einer Operation oder bei Immobilität.

Bei Nierenerkrankungen (z. B. Pyelonephritis) kann der Urin zuverlässig und konstant abgeleitet werden.

Vorteil dieses Katheters ist die einfache Anlage und die vergleichsweise wenigen Kontraindikationen. Nachteil ist, dass es bei einem lang liegenden Katheter und unzureichender Hygiene zu Infektionen kommen kann [8].

Komplikationen bei der Anlage eines transurethralen Katheters reichen von Verletzungen der Harnröhre bis hin zu Verstopfung des Katheters, Harnröhrenstrikturen, aufsteigende Infektionen, Harnblasensteine oder Via Falsa [2].

Kontraindikationen sind urologische Infektionen der ableitenden Harnwege wie Prostatitis und Epididymitis, Harnröhrenengen sowie Harnröhrenverletzungen. Hierbei muss auf einen suprapubischen Katheter zurückgegriffen werden [2].

\subsubsection{Suprapubischer Blasenkatheter}

Ein suprapubischer Blasenkatheter (SPK) stellt eine Möglichkeit dar, den Urin des Patienten mittels Punktion dauerhaft über die Bauchdecke aus der Harnblase abzuleiten. Somit ist es möglich die physiologische Harnableitung über die Harnröhre zu umgehen.

Indikation für einen suprapubischen Katheter sind z. B. die Harnableitung nach einem Eingriff an den ableitenden Harnwegen, bei frustranem Versuch einer transurethralen Katheteranlage oder bei einer Beckenfraktur.

Ebenfalls wird in den EAU-Leitlinien darauf hingewiesen, dass bei Infektionen der Prostata und des Nebenhoden, die mit einer Harnretention einhergehen, eine suprapubische Harnableitung erforderlich ist [1].

Vorteil des SPK ist die einfachere Hygiene, die bessere Mobilität und Verträglichkeit der Patienten sowie seltener schwerwiegende Komplikationen. AuBerdem reduziert sich durch die Anlage eines suprapubischen Katheters das 
weitere Infektionsrisiko für Prostata und Nebenhoden, durch Verhinderung einer Restharnbildung mit Keimreservoir [1].

Komplikationenen beim SPK können perivesikale oder intravesikale Blutungen, Fehlpunktionen, Verletzungen von Darm oder Gefäßen, Nachblutungen und Infektionen sein [2].

Kontraindikationen sind Tumoren im Urogenitaltrakt oder Unterbauch, Blutgerinnungsstörungen, Hauterkrankungen im Punktionsbereich sowie eine verlagerte oder kleinkapazitäre Harnblase. Bei Patienten mit starker Vernarbung durch z. B vorrangegangene Operationen sollte ebenfalls auf die Anlage verzichtet werden [2].

\section{Studiendesign/-durchführung \\ 2.1 Studiendesign}

Es handelt sich um eine klinisch-randomisierte Studie, die auf ein Jahr ausgelegt ist. Die Patientendaten werden pseudonymisiert.

Insgesamt sollen in der Studie 100 Patienten untersucht werden, die in 2 Arme (Harnableitung vs. Keine Harnableitung) randomisiert werden. Zusätzlich sollen retrospektive Daten von Patienten der letzten Jahre mit eingepflegt werden, um eine bessere Vergleichbarkeit zu erzielen.

Zusätzliche Diagnostik der Studienteilnehmer:

Die Patienten der Studie werden einmal zusätzlich am 3. Tag nach Aufnahme eine Urinprobe abgeben und die Männer erhalten nach dem sie in die Studie eingewilligt haben bei Aufnahme zusätzlich einen Harnröhrenabstrich. Im Laufe der Studie werden die Teilnehmer zur Aufnahme sowie Entlassung einen Quality of Life-Fragebogen sowie eine Analogsklala ausfüllen.

Die weitere Diagnostik entspricht dem klinischen Standard der UMG. Dies beinhaltet ein Ausgangslabor mit Blutbild, Elektrolyten, CRP und Quick/PTT, PCT und PSA, ggf. eine DRU sowie ggf. ein TRUS (bei Männern), eine Temperaturmessung, die Abnahme von Blutkulturen bei Fieber und ggf. einen Urinstatus.

Studienbedingte Maßnahmen werden erst nach dem Einverständnis der Probanden erfolgen.

Therapiert werden alle männlichen Patienten mit dem Fluorchinolon Ciprofloxacin 500 mg 1-0-1 über 14 Tage. Patienten unter 45 Jahren erhalten zusätzlich Doxycyclin 400 mg 1-0-1. Frauen mit Pyelonephritis werden für 14 Tage entweder oral mit Cefpodoxim oder Alternativ mit einer intravenösen Cephalosporintherapie (Ceftriaxon oder Cefotaxim) 1-0-1 behandelt. Falls sich bei Patienten ohne Harnableitung nach 3 Tagen keine Besserung hinsichtlich Klinik oder Labor einstellt, wird den Patienten ebenfalls ein Katheter gelegt. Dem Patienten wird somit in keiner Weise eine mögliche Therapie vorenthalten.

Nachdem die Patientendaten über ein Jahr erfasst worden sind, werden diese anhand der gelaufenen Diagnostik ausgewertet. Um eine bestmögliche Beurteilung der verschiedenen Therapieverfahren zu gewährleisten, werden die männlichen Probanden einem Follow-Up unterzogen. Sie bekommen bei Ent- 
lassung einen Termin zur Wiedervorstellung 5 Tage nach Ende der Antibiotikatherapie. Hier wird nach Durchführung einer Abschlussuntersuchung, Laboruntersuchung, Ausfüllen des SF-12 (s.u.), Uroflow und Restharnbestimmung ggf. der SPK entfernt.

Den Frauen wird nach 48 Std. Fieberfreiheit der transurethrale Katheter gezogen. Vor Entlassung werden eine Laboruntersuchung, körperliche Abschlussuntersuchung, Restharnbestimmung und Erfassung des SF-12 (s.u) durchgeführt.

$\mathrm{Zu}$ einer besseren Vergleichbarkeit sollen zusätzlich retrospektive Daten von Patienten /Patientinnen erfasst werden, die in der UMG unter der Diagnose akute Prostatitis, Epidydimitis und akute Pyelonephritis der Frau in den letzten Jahren behandelt worden sind.

Ziel ist es, bei fortlaufender prospektiver Studie, unser Studienziel von 100 Studienpatienten zu erreichen.

Es werden lediglich Daten erfasst, die bereits während des stattgehabten Krankenhausaufenthaltes erhoben worden sind. Dazu zählen die Aufenthaltsdauer im Krankenhaus, der Temperaturverlauf, das Alter der Patienten, die Laborwerte (BB, CRP, ggf. PCT) bei Aufnahme sowie Entlassung, mögliche Ergebnisse von Urinkontrollen sowie die Information, ob ein Katheter gelegt worden ist oder nicht.

Die Daten werden anonym ausgewertet und zur besseren Vergleichbarkeit mit in die Ergebnisse der Studie eingepflegt. Für die Patienten ergibt sich dadurch kein Nachteil.

\subsection{Studiendurchführung sowie Risiko und Nutzen Abwägung}

Nachdem die Diagnose von dem diensthabenden Arzt gestellt wurde, werden die Patienten über die Studie aufgeklärt und stationär aufgenommen. Die Einverständniserklärung liegt dem Ethikantrag bei.

Im Anschluss werden alle Kontraindikationen und Ausschlusskriterien überprüft und es kommt zur Randomisierung des Patienten. Es folgt bei beiden männlichen Gruppen (Katheter vs kein Katheter) die Abnahme eines studienbedingten Harnröhrenabstriches bevor ggf. der suprapubische Katheter gelegt wird.

Nach dem Abstrich beginnt die kalkulierte Antibiotikagabe wie oben beschrieben.

Am 3. Tag nach Aufnahme werden die Probanden studienbedingt eine zusätzliche Urinprobe abgeben.

Während des Krankenhausaufenthaltes werden die Probanden einmal zu Beginn und zur Entlassung zu ihren Schmerzen anhand einer visuellen Analogskala befragt und werden dazu einen Quality of Life-Fragebogen (SF-12) ausfüllen.

Dieser Fragebogen ist als Kurzform des SF-36-Fragebogens ein „krankheitsübergreifendes Messinstrument zur Erfassung der subjektiven gesundheitsbezogenen Lebensqualität“" [12]. Dieser umfasst die Einschätzung der Körperli- 
chen Funktion, der Schmerzen, der Vitalität, die soziale und emotionale Rolle, das psychische Wohlbefinden und die Allgemeine Gesundheitswahrnehmung [11] [12].

Zur Auswertung der Studie werden neben dem Labor die Ergebnisse des Quality of life Fragebogens (SF-12), die visuelle Analogskala, die Länge des Krankenhausaufenthaltes, der Schmerzmittelverbrauch und eventuelle Komplikationen erfasst.

Entlassen werden die Patienten nach 48 Stunden Fieberfreiheit und CRPWerten $<100 \mathrm{mg} / \mathrm{dl}$.

Wie in den Leitlinien beschrieben werden die Patienten 14 Tage mit Antibiotika behandelt [1] [10].

5 Tage nach Absetzen der Antibiotika erfolgt eine Follow-Up-Untersuchung bei den männlichen Probanden der Kathetergruppe. Hier wird die suprapubische Harnableitung entfernt; zudem werden eine Uroflowmetrie, sowie einen Ulltraschall zur Restharnbestimmung durchgeführt. Hierfür ist es notwendig, dass die Patienten am 4. Tag den SPK abstöpseln und in dem mitgegebenen Miktionsprotokoll die Restharnmengen dokumentieren, da sich so der Restharn genauer bestimmen lässt. Außerdem werden die Patienten beim Follow-Up ein letztes Mal den SF-12-Fragebogen ausfüllen.

\section{Risiko-Nutzen-Abwägung:}

Das Risiko für die Patienten erstreckt sich von Harnröhrenverletzungen, Fehlpunktionen und Nachblutungen bis hin zu Infektionen durch die Katheteranlage.

Der Patient selber hat keinen direkten Nutzen von der zusätzlich abzugebenden Urinprobe, dem Harnröhrenabstrich oder der erhobenen Daten des SF12-Fragebogens.

Von Vorteil für den Patienten ist möglicherweise, dass durch den suprapubischen bzw. transurethralen Katheter sichergestellt werden kann, dass es zu keiner Restharnentstehung in der Blase kommt. Diese stellt eine potenzielle Keimquelle dar, welche zu einem komplizierteren Verlauf der Erkrankung beitragen könnte.

Die im Rahmen der Dokumentation zusammengefassten Ergebnisse können ggf. neue Erkenntnisse für die Therapie zukünftiger Patienten beinhalten und somit die therapeutische Herangehensweise bei Prostatitis/Epididymitis und Pyelonephritis der Frauen verändern.

\subsection{Patienten/Probandenrekrutierung}

\subsubsection{Anzahl der Patienten, Einschlusskriterien und Dauer der Studie.}

In die Studie sollen insgesamt 100 Patienten eingeschlossen werden.

Eingeschlossen werden nur volljährige und einwilligungsfähige Patienten die sich mit Prostatitis, Epididymitis oder Pyelonephritis bei der Frau in stationärer Behandlung in der Urologischen Klinik der Universitätsmedizin Göttingen (UMG) befinden und keine der unten beschriebenen Kontraindikationen für einen Katheter aufweisen. 
Durch Randomisierung über eine standardisierte Randomisierungsliste wird ausgewählt, welche der 100 Patienten einen Katheter bekommen.

Diese Liste ordnet per Zufallsverteilung die Patienten entweder der Kathetergruppe oder der Kontrollgruppe zu, sodass am Ende der Studie zwei gleich große Kohorten entstehen. Die Liste wird dem ärztlichen Personal vorgelegt.

Zusätzlich werden retrospektive Daten von Patienten/Patientinnen erfasst, die sich in stationärer Behandlung in der UMG befanden. Hierdurch soll eine bessere Vergleichbarkeit sowie eine weitere Perspektive mit einbezogen werden.

\subsubsection{Auswahl der Probanden}

Das Ziel ist es jeweils 50 Probanden mit Prostatitis/Epididymitis und 50 Probandinnen mit Pyelonephritis in diese Studie einzuschließen. Es werden nur volljährige und einwilligungsfähige Patienten eingeschlossen. 25 Patienten jeder Gruppe bekommen randomisiert einen Katheter, die anderen jeweils 25 Patienten bilden die Kontrollgruppe.

Die Gesamtzahl von 100 Probanden wird nicht überschritten.

Die Anzahl der Patienten die nach einem Jahr nicht erfasst wurden wird mit retrospektiven Daten der UMG ergänzt.

\subsection{Ausschlusskriterien}

Ausgeschlossen werden Patienten mit jeglicher Kontraindikation für einen Katheter. Hierzu zählen Tumoren im Unterbauch, Blutgerinnungsstörungen (ggf. Antikoagulantientherapie), eine kleinkapazitäre Harnblase oder Hauterkrankungen und starke Vernarbungen im Punktionsbereich. Harnröhrenverletzungen sowie Strikturen sind ein Ausschluss für eine transurethrale Harnableitung.

Bei den Frauen stellt zusätzlich eine Schwangerschaft ein Ausschlusskriterium dar.

Außerdem werden Patienten von der Studie ausgeschlossen, die eine relevante Restharnmenge von > $300 \mathrm{ml}$ aufweisen und somit aufgrund einer Harnabflussstörung in jedem Fall einen Katheter benötigen. Dieses medizinische Vorgehen entspricht dem klinischen Standard der UMG.

Patienten, bei denen aus medizinischer Indikation ein Dauerkatheter gelegt werden muss, können im Rahmen der Studie nicht Randomisiert werden. Hier ist nach Einverständnis der Patienten dennoch eine Erhebung der Daten mit anschließender Auswertung geplant (Siehe beiliegende Einverständniserklärung und Patienteninformation).

Die erhobenen Daten werden in einer Datenbank gespeichert und einer statistischen Analyse mit dem Programm Statistica unterzogen. Bei evtl. Fragen zur statistischen Bewertung der Arbeit ist eine Zusammenarbeit mit der Abteilung Medizinische Statistik der Georg-August-Universität Göttingen vorgesehen.

\section{Archivierung und Datenschutz}

Die Daten werden für 10 Jahre gespeichert und anschließend vernichtet. Eine Weitergabe an dritte Personen wird nicht erfolgen. 
Den Patientendaten sowie den Fragebögen und Urinproben wird eine Nummer zugeordnet, die keinen Rückschluss auf die persönlichen Daten des Patienten erlaubt. Die Zuordnung der Nummern erfolgt in einer Excel-Datei, die in einem passwortgeschützen Computer der Studienleitung hinterlegt wird. Die Rückführung der gewonnen Ergebnisse auf persönliche Daten ist somit nur der Studienleitung möglich. Nach Abschluss der Studie wird diese Datei vernichtet.

\section{Literaturverzeichnis}

[1] „EAU-Guidelines-Urological-Infections-v2.pdf.”; http://uroweb.org/wpcontent/uploads/EAU-Guidelines-Urological-Infections-v2.pdf aufgerufen am 21. Januar 2016, S 42-47.

[2] „Katheter und Drainagen“ http://www.urologielehrbuch.de/transurethraler_dauerkatheter.html aufgerufen am 07.03.2016

[3] H-U.Schmelz, C. Sparwasser, W. Weidner; Facharztwissen Urologie: Differenzierte Diagnostik und Therapie. Springer Verlag, 2011, 105-107.

[4] "Akute Prostatitis: Bakterielle Prostataentzündung." http://www.urologielehrbuch.de/akute_prostatitis.html aufgerufen am 24 Januar 2016.

[5] K. Aktories, U. Förstermann, F.B. Hoffmann; Allgemeine und Spezielle Pharmakologie und Toxikologie. Elservier $\mathrm{GmbH}$ München, Auflage 11, 2013, S.793-795

[6] R.E. Hautmann, H. Hartwig; Urologie. 2. vollst. überarb. Aufl. Berlin Heidelberg New York: Springer Verlag, 2000, S.164-171.

[7] „Epididymitis (Nebenhodenentzündung)." http://www.urologielehrbuch.de/akute_epididymitis.html aufgerufen am 24 Januar 2016

[8] "Nierenbeckenentzündung (Akute Pyelonephritis)." http://www.urologielehrbuch.de/akute_pyelonephritis.html aufgerufen am 24 Januar 2016.

[9] W. Merkle; Urologie. Stuttgart: Hippokrates Verlag GmbH, 1997, S. 133-136.

[10] "EAU-Guidelines-Urological-Infections-v2.pdf." http://uroweb.org/wpcontent/uploads/EAU-Guidelines-Urological-Infections-v2.pdf aufgerufen am 21 Januar 2016, 16-18.

[11] http://www.mlbk.de/web/html/quellen/sf-12.html; aufgerufen am 07.03.2016

[12] http://www.testzentrale.de/programm/sf-36-fragebogen-zumgesundheitszustand.html; aufgerufen am 7.03.2016 


\section{Votum Ethik-Kommission}

\section{UNIVERSITÄTSIMEDIZIN $=1 \mathrm{MAO}$
GOTTINGEN $=0$}

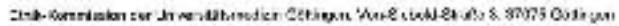

Herm

Dr. med. Oliver Hahn

Klinik für Urologie

Robert-Koch-St. 40

vcrabs per E-Wail: cliver.hahn@imed.unf-goettingen de
Ethik-Konmistion der Universităsmedizin Gottingen Vorsizenker: Pior. Dr. Jurgen Erockmëller Referentin Regierungsratin Doris Wettschereck NEU 0551 : 39-68544 Telefon

Von-Siebold-Straße 3, 37076 Göttingen Adresece NEU 0551 ; 3 H-R.281 Teleton NEU $0551 / 39-E 9536$ Fax ethik@med. uni-goettingen,de E-Mail wnw.ethikkommission med uni-goettingen.de 06.05.2017 br - gö Datum
Antragsnummer:

Studientitel:

Antragsteller:
25/4/16 (bitte stets angeben)

Unterguchung des Stellemvarta won trarsurgth raler und suprapubischer Hamatsleilung in der Therapie won Prosiatilis, Epididymitis und Pyelonephntis"

Kurztitel: Dia Rolle der Harnableitung in therapeulisenen Management kcmplizierter Harnwegsinfeklicnen

Dr. mad. Oliver Hahn, PO Dr. med. Sastra Ahyei, Prol. Dr, med. Lulz Trajan. Marlena Stabein. Klinik fur Urologie, UMC

Sehr geehrter Herr Dr. Hahn, sehr geehrte Damen und Herren.

fưr die oben genannte Studia hat die Ethikkomrnission mit Schreiben vom 17.03.2017 ein pos itves Votum erteilt.

Eezöglich einiger nachträglicher Änderungen Ihrerseits haben Sie mit Schreiben vom 28.03 .17 weitere Erläuterungen und Erganzungen vorgelegt. Es bestehen keine Bedenken gegen diese nachtraglichen Anderungen. Damit bestahen also auch keine Bedenken gegen die Durchfürung der Slucie gemäß Ihrem mit Schrelben vom 28.03.2017 vorgelegten Studenprotokol.

Wir wïnschen weiterhin viel Erfolg bel der Studle.

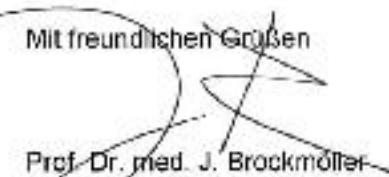

vors itzender der Ethik-Kommission-

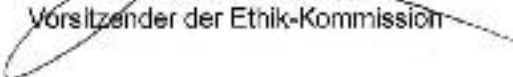




\section{Patienteneinverständniserklärung}

Klinik für Urologie

Direktor: Univ.-Prof. Dr. med. L. Trojan

Robert-Koch-Str. 40, 37075 Göttingen

Klinik für Urologie, Universitätsmedizin Göttingen

\section{Patienteneinverständniserklärung}

zur Studie

Untersuchung des Stellenwerts von transurethraler und suprapubischer Harnableitung in der Therapie von Prostatitis, Epididymitis und Pyelonephritis

Ich, .(Name, Vorname) wurde von meinem Arzt über Wesen, Bedeutung und Tragweite der klinischen Studie mit dem o.g. Titel aufgeklärt.

Ich habe den Aufklärungstext gelesen und verstanden. Ich hatte die Möglichkeit, Fragen zu stellen, habe die Antworten verstanden und akzeptiere sie. Mein Arzt hat mich über die mit der Teilnahme an der Studie verbundenen Risiken und den möglichen Nutzen aufgeklärt.

Ich hatte genug Zeit über die Teilnahme an der Studie nachzudenken und weiß, dass diese Teilnahme freiwillig ist.

Ich weiß auch, dass ich zu jeder Zeit und ohne Angabe von Gründen diese Einwilligung zur Studie wiederrufen kann, ohne dass sich diese Entscheidung auf die spätere medizinische Behandlung durch meinen Arzt auswirkt.

Mir ist bekannt, dass meine persönlichen Daten für 10 Jahre in verschlüsselter Form gespeichert werden. 
Mit meinem Einverständnis zur Teilnahme erkläre ich gleichzeitig, dass ich mit der im Rahmen dieser klinischen Studie erfolgenden Aufzeichnung von Krankheitsdaten einverstanden bin.

Mir ist bekannt, dass im Rahmen dieses Forschungsvorhabend personenbezogene Daten erhoben und in pseudonymisierter (verschlüsselter) Form aufgezeichnet und gespeichert werden.

Die Datenspeicherzeit beträgt 10 Jahre. Die Personenbezogenen Daten werden nicht an Dritte weitergegeben. Ich weiß, dass ich mein Einverständnis zur Speicherung der personenbezogenen Daten jederzeit widerrufen kann. Im Falle des Widerrufs werden alle personenbezogenen Daten gelöscht.

Ich habe eine Kopie der Patienteninformation und dieser Einwilligung erhalten. Ich erkläre hiermit meine freiwillige Teilnahme an dieser klinischen Studie. 


\title{
4. Patienteninformation
}

\section{Patienteninformation}

\author{
zur Studie:
}

\section{Untersuchung des Stellenwerts von transurethraler und suprapubischer Harnableitung in der Therapie von Prostatitis, Epididymitis und Pyelonephritis}

Sehr geehrter Patient,

diese Patienteninformation soll Sie über eine wissenschaftliche Studie aufklären, die wir in der Urologischen Abteilung der Universitätsmedizin Göttingen durchführen. Hierbei wird durch eine Zufallsverteilung die Hälfte der Patienten mit einer Prostataentzündung oder Nebenhodenentzündung mit einem suprapubischen Blasenkatheter versorgt. Dieser Katheter wird direkt über die Bauchdecke in die Harnblase geführt und gewährleistet eine konstante Harnableitung. Im Falle einer Prostata-/ Nebenhodenentzündung ist es wichtig eine Restharnbildung in der Blase zu verhindern, um ein aufsteigen von weiteren Keimen zu unterbinden. Grundsätzlich kann es bei einer Katheteranlage zu Infektionen kommen, im schlimmsten Fall zu einer Verletzung der Harnblase. Diese Komplikationen sind allerdings sehr selten. Ein mittels der Studie zu überprüfender Vorteil dieses Eingriffs ist eine Verringerung der Komplikationen während der Erkrankung, eine schnellere Genesung und somit ein schnelleres Wiedereingliedern in den Alltag, dadurch das der Urin nicht an der infizierten und schmerzhaften Stelle vorbeiläuft. Die Risiken für die Katheteranlage entnehmen sie dem standadisierten Aufklärungsbogen.

Im Verlauf Ihres Krankenhausaufenthaltes kommt es zur routinemäßigen Diagnostik und Therapie ihrer Erkrankung. Zusätzlich benötigen wir nach der Aufnahme einen Harnröhrenabstrich und am 3. Tag eine zusätzliche Urinprobe, die wird über den gelegten Katheter abnehmen und die von uns im Anschluss analysiert wird. Auf diese Weise ist es für uns möglich, das Vorhandensein von Erregern bzw. das Ansprechen der Therapie zu überprüfen. Im Verlauf ihrer Behandlung werden sie gebeten einen „Quality of Life“-Fragebogen auszufüllen, damit wir ihr allgemeines Befinden dokumentieren und vergleichen können.

Nach ihrer Entlassung bekommen sie einen Wiedervorstellungstermin, an dem der Katheter gezogen wird und wir eine abschließende Untersuchung machen. So können wir den Verlauf der Behandlung exakt dokumentieren.

Insgesamt ist unsere Studie über 1 Jahr Dauer ausgelegt.

Ihre personenbezogenen medizinischen Daten verbleiben ausschließlich in der Hand ihrer betreuenden Ärzte. Für die Auswertung der Studie werden ihre Daten ausschließlich pseudonymisiert verwendet, d.h. ihr Name wird darin nicht auftauchen. 
Ihre Daten werden mit einer für die durchführenden Ärzte erkennbare Nummer gekennzeichnet, die nicht auf Sie zurückschließen lässt.

Ihre Daten werden im Rahmen unserer Studie für 10 Jahre gespeichert und im Anschluss vernichtet. Es erfolgt zu keinem Zeitpunkt eine Weitergabe an dritte Personen.

Durch Ihre Teilnahme an dieser Studie ändert sich nichts an ihrer medizinischen Behandlung. Bevor Sie sich zur Studienteilnahme entschließen, müssen Sie die Einverständniserklärung „Untersuchung des Stellenwerts von transurethraler und suprapubischer Harnableitung in der Therapie von Prostatitis, Epididymitis und Pyelonephritis der Frau“ gelesen und mit ihrem Arzt besprochen haben.

Ihre Teilnahme ist freiwillig. Sie können jederzeit und ohne Angabe von Gründen Ihre Teilnahme wiederrufen, ohne dass Ihnen Nachteile in der ärztlichen Versorgung entstehen. Im Falle eines Wiederrufes werden alle gespeicherten Daten vernichtet.

Sie würden mit ihrer Bereitschaft zur Teilnahme an dieser Studie zur Weiterentwicklung des therapeutischen Vorgehens bei Prostata- und Nebenhodenentzündung beitragen, so dass in Zukunft möglicherweise andere Patienten von der veränderten Therapie profitieren.

Als Prüfärzte sind für Sie ansprechbar:

- Prof. Dr. Lutz Trojan

- PD Dr. Sascha Ahyai

- Dr. Arne Strauß

- Dr. Florian Pütsch

- Dr. lonel Valentin Popeneciu

- Fr. Simone Braun

- Hr. Lucas Fischer

- Fr. Maria Gerling

- Dr. Oliver Hahn

- Dr. Felix Kessel

- Hr. Markus Krohm

- Hr. Conrad Leitsmann

- Hr. Florian Meier

- Hr. Johannes Neumann

- Dr. Marianne Schmid

- Fr. Justina Traceviciute

- Hr. Jan Lüdecke

- Dr. Annemarie Uhlig

Kontakt über das Sekretariat der Klinik für Urologie 


\section{Fließschema Männer- Patientenmappe}

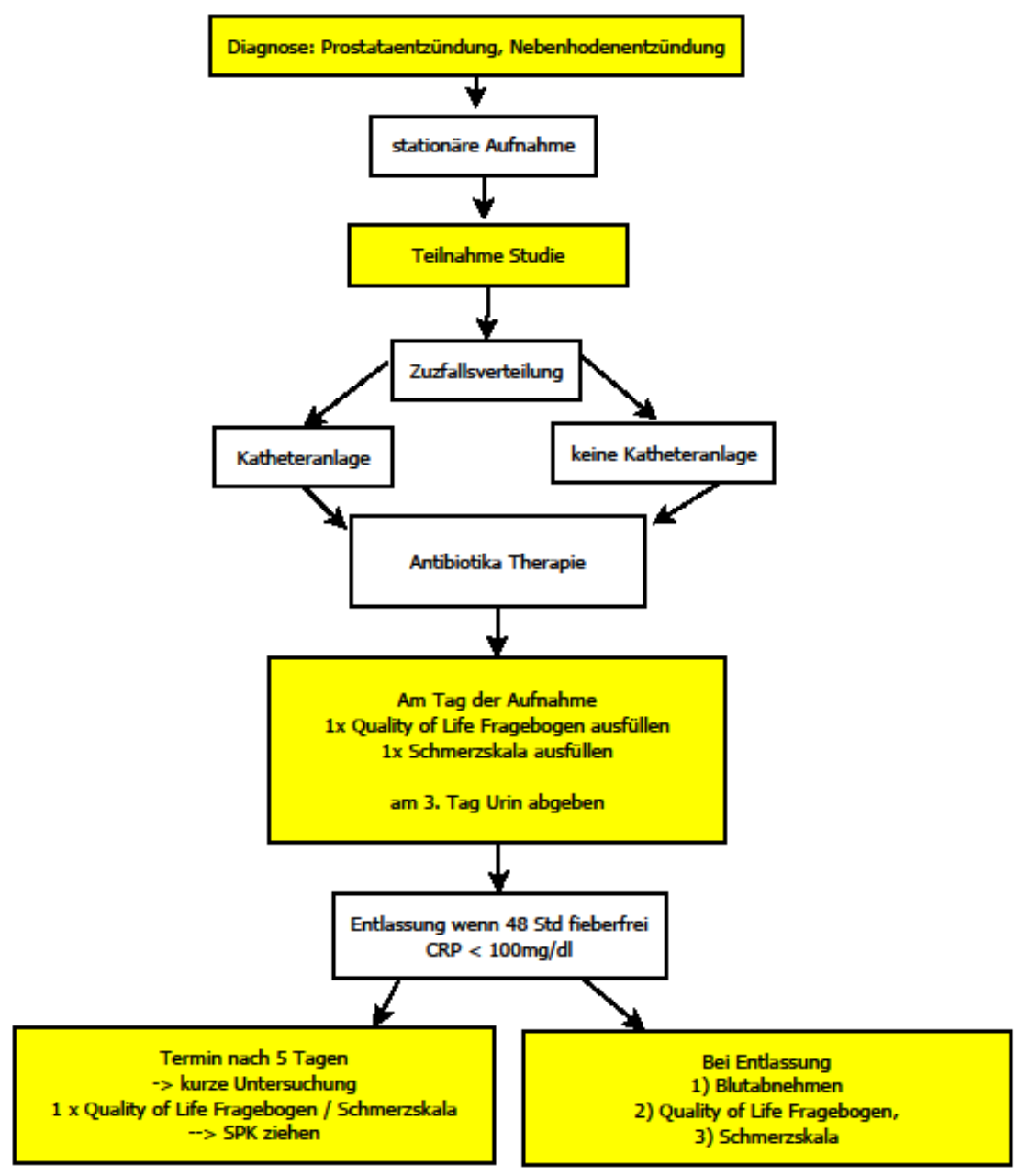




\section{Fließschema Frauen-Patientenmappe}

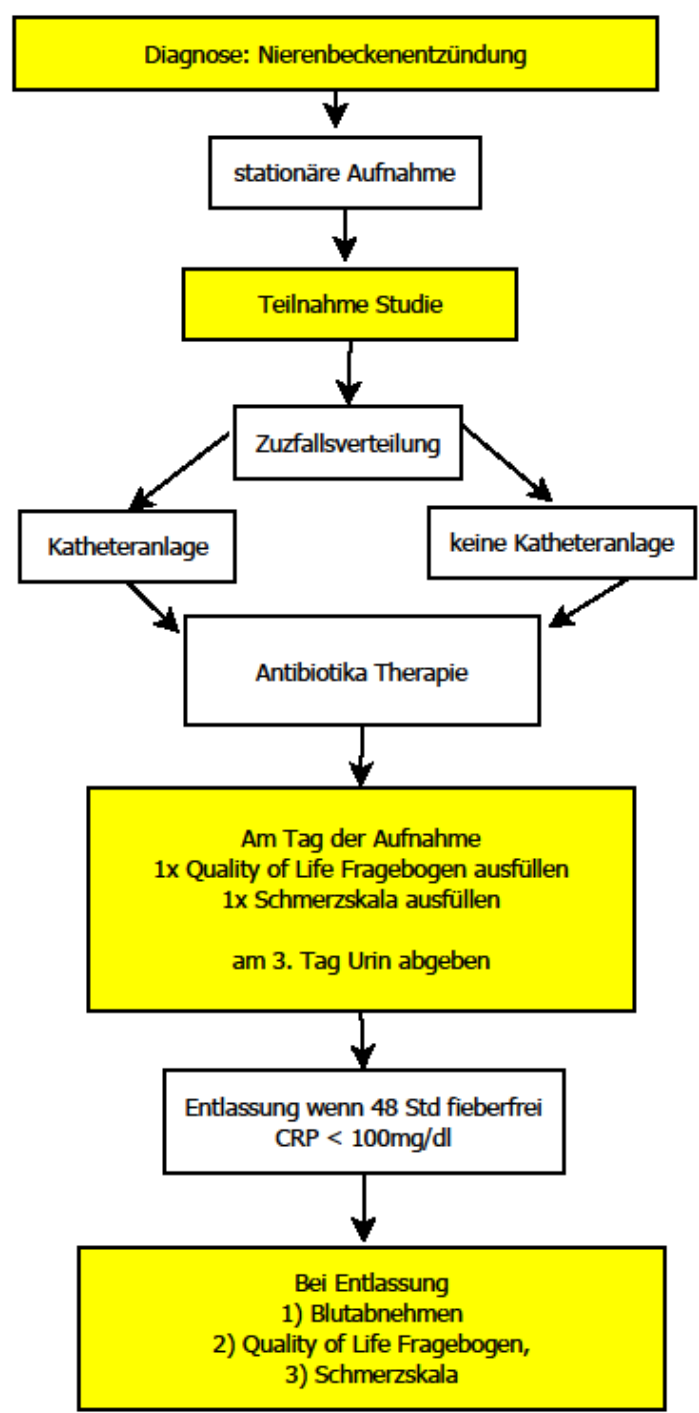




\section{SF-12-Fragebogen}

\section{UNIVERSITÄTSMEDIZIN $=1 / \mathbf{G}$
GOTTINGLN $=\mathbf{W}$}

Gesundheitsfragebogen SF-12 (short form)

Klinik für Urologie

Fragebogen zur gesundheitsbezogenen Lebensqualität Direktor. Univ.-Prof. Dr. med. L. Trojan

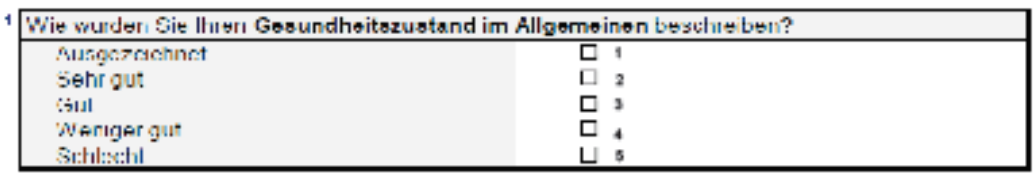

\begin{tabular}{|c|c|c|c|}
\hline \multirow{4}{*}{ 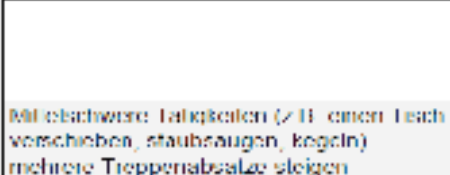 } & $\begin{array}{l}\text { Jo, } \\
\text { ilialk. } \\
\text { singand.hran }\end{array}$ & $\begin{array}{l}\text { IA. } \\
\text { clws:; } \\
\text { congenthrankt }\end{array}$ & $\begin{array}{l}\text { Nain, } \\
\text { ubisthaug: ruk:hl } \\
\text { cingceshorarikt }\end{array}$ \\
\hline & , & 2 & , \\
\hline & $\square$ & 口 & ㅁ \\
\hline & п & 峛 & П \\
\hline
\end{tabular}

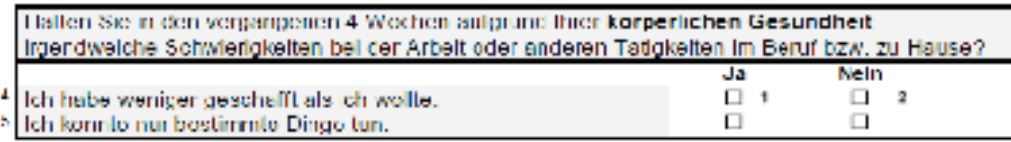

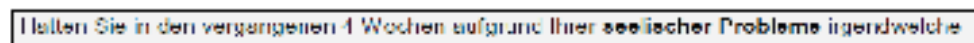

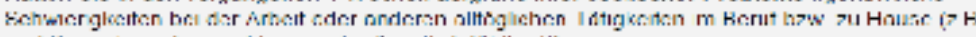

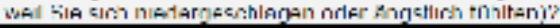

Ich heba wenlser jeschefft als ch wolte.

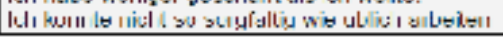

ㅁ $\square$

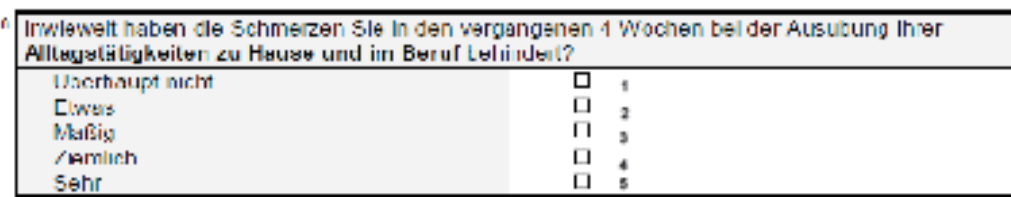

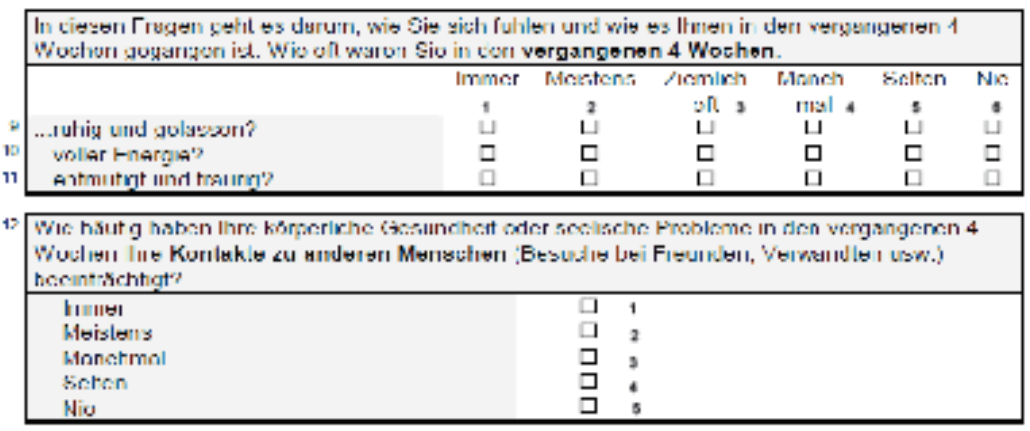




\section{Literaturverzeichnis}

Aktories K, Forth W: Allgemeine und spezielle Pharmakologie und Toxikologie. 11. überarb. Auflage; Elsevier Urban \& Fischer, München 2013

Albrecht K, Oelke M, Schultheiss D, Tröger HD (2004): Die Relevanz der Harnblasenfüllung bei der suprapubischen Blasenkatheterisierung. Urologe A $\underline{43}, 178-184$

Altman DG, Gore SM, Gardner MJ, Pocock SJ (1983): Statistical guidelines for contributors to medical journals. Br Med J (Clin Res Ed) 286(6376), 1489-1493

AWMF-Leitlinie unkomplizierte Harnwegsinfektionen 2017: Interdisziplinäre S3Leitlinie: Epidemiologie, Diagnostik, Therapie, Prävention und Management unkomplizierter bakterieller, ambulant erworbener Harnwegsinfektionen bei erwachsenen Patienten. https://www.awmf.org/uploads/tx_szleitlinien/043-

044k_S3_Harnwegsinfektionen_2017-05.pdf Zugriff am: 1.03.2018

AWMF-Leitlinie Harndrainage 2015,https://www.awmf.org/uploads/tx szleitlinien/029007I S1 Harndrainage 2015-02 01.pdf Zugriff am 9.11.2017

Bergner M, Bobbitt RA, Carter WB, Gilson BS (1981): The Sickness Impact Profile: Development and final revision of a health status measure. Med Care 19(8), 787-805

Berth H, Balck F: Psychologische Tests für Mediziner. 1. Auflage; Springer, Berlin Heidelberg 2003

Bestmann B (2015): Messung von Lebensqualität. https://www.tk.de/centaurus/servlet/contentblob/749464/Datei/2746/Summer-School2015.pdf Zugriff am 12.11.2017

Blacklock NJ (1991): The anatomy of the prostate: Relationship with prostatic infection. Infection $\underline{19}$ Suppl 3, 111-114

Bleidorn J, Gágyor I, Kochen MM, Wegscheider K, Hummers-Pradier E (2010): Symptomatic treatment (ibuprofen) or antibiotics (ciprofloxacin) for uncomplicated urinary tract infection? - Results of a randomized controlled pilot trial. BMC Med $\underline{8}, 30$

Bonkat G, Priller JM, Müller G, Subotic S, Bachmann A (2011): Suprapubische Harnableitung. https://www.tellmed.ch/include php/previewdoc.php?file id=7300, Zugriff am 21.12.2017

Bubeck J: Urologische Implantate. In: Jocham D, Miller K (Hrsg.): Praxis der Urologie Band 1; Thieme, Stuttgart New York 1994, 199-209

Çek M, Sturdza L, Pilatz A (2017): Acute and Chronic Epididymitis. Eur Urol Suppl $\underline{16}$ (4), 124-131

Cella D, Riley W, Stone A, Rothrock N, Reeve B, Yount S, Amtmann D, Bode R, Buysse D, Choi S (2010): The Patient-Reported Outcomes Measurement Information 
System (PROMIS) developed and tested its first wave of adult self-reported health outcome item banks. J Clin Epidemiol 63 (11), 1179-1194

Collins MM, Meigs JB, Barry MJ, Corkery EW, Giovannucci E, Kawachi I (2002): Prevalence and correlates of prostatitis in the health professionals follow-up study cohort. J Urol 167, 1363-1366

Davis NG, Silberman M. Bacterial acute Prostatitis. [Updated 2019 Feb 28] In: StatPearls [Internet]. Treasure Island (FL). https://www.ncbi.nlm.nih.gov/books/NBK459257/\#article-27857.s3 Zugriff am 20.02.2018

Dietz O (1960): The change in the degree of fertility during the course of acute nonspecific epididymitis. (Contribution to the pathogenesis of primary inhibition of spermiogenesis). Arch Med Infant 211, 160-166

Draper NR, Smith H: Applied regression analysis (Wiley series in probability and statistics Texts and references section). 3. Auflage; Wiley, New York NY u.a. 1998

Du Prel J-B, Röhrig B, Hommel G, Blettner M (2010): Choosing statistical tests: Part 12 of a series on evaluation of scientific publications. Dtsch Arztebl Int 107, 343-348

Duller C: Einführung in die nichtparametrische Statistik mit SAS und R: Ein anwendungsorientiertes Lehr- und Arbeitsbuch. Physica-Verlag, Heidelberg 2008

EAU-Guidelines Urological Infections-Prostatitis/Epididymitis 2015, 42-47 http://uroweb.org/wp-content/uploads/EAU-Guidelines-Urological-Infections-v2.pdf; Zugriff am 15.01.2018

EAU-Guidelines Urological Infections-Pyelonephritis 2015, 16-18 http://uroweb.org/wp-content/uploads/EAU-Guidelines-Urological-Infections-v2.pdf; Zugriff am 15.01.2018

Efstathiou SP, Pefanis AV, Tsioulos DI, Zacharos ID, Tsiakou AG, Mitromaras AG, Mastorantonakis SE, Kanavaki SN, Mountokalakis TD (2003): Acute pyelonephritis in adults: Prediction of mortality and failure of treatment. Arch Intern Med 163, 12061212

Esposito S, Noviello S, Leone S (2008): [catheter-associated urinary tract infections: epidemiology and prevention]. Infez Med 16, 130-143

Etienne M, Chavanet P, Sibert L, Michel F, Levesque H, Lorcerie B, Doucet J, Pfitzenmeyer P, Caron F. (2008): Acute bacterial prostatitis: Heterogeneity in diagnostic criteria and management. Retrospective multicentric analysis of 371 patients diagnosed with acute prostatitis. BMC Infect Dis $\underline{8}, 12$

Etienne M, van Elslande H, Choplin-Renard J, Pestel-Caron M, Caron F (2014): Antibiotic resistance in adult female patients hospitalized for acute pyelonephritis: Rates and predicting factors. Med Mal Infect $\underline{44}, 217-222$ 
Fahrmeir L, Kneib T, Lang S: Regression: Modelle, Methoden und Anwendungen (Statistik und ihre Anwendungen). 2. Auflage; Springer-Verlag, Berlin Heidelberg 2009

Foxman B, Frerichs RR (1985): Epidemiology of urinary tract infection: I. Diaphragm use and sexual intercourse. Am J Public Health $\underline{75}$, 1308-1313

Füssle R, Sziegoleit A: Praxis der Infektiologie: Organbezogene Diagnostik und Therapie. Springer, Berlin Heidelberg 2001

Gágyor I, Hummers-Pradier E, Kochen MM, Schmiemann G, Wegscheider K, Bleidorn J (2012): Immediate versus conditional treatment of uncomplicated urinary tract infection - a randomized-controlled comparative effectiveness study in general practices. BMC Infect Dis $\underline{12}, 146$

Gágyor I, Bleidorn J, Kochen MM, Schmiemann G, Wegscheider K, HummersPradier E (2015): Ibuprofen versus fosfomycin for uncomplicated urinary tract infection in women: Randomised controlled trial. BMJ 351, 1-11

Gujadhur R, Aning J (2015): Careful assessment key in managing prostatitis. Practitioner $\underline{259}, 15-19$

Harper A, Power M (1998): Development of the World Health Organization WHOQOL-BREF quality of life assessment. The WHOQOL Group. Psychol Med $\underline{28}$, $551-558$

Hautmann R: Urologie (Springer-Lehrbuch). 4., überarbeitete und aktualisierte Auflage; Springer-Verlag, Berlin Heidelberg 2010

Hengesbach S, Hinkelbein J, Genzwürker H, Neuhaus C, Kollrack YMB, Knauß A: Checkliste Medical Skills: 34 Tabellen (Checklisten der aktuellen Medizin). Georg Thieme-Verlag, Stuttgart New York 2013

Holling H: Handbuch Statistik, Methoden und Evaluation (Handbuch der Psychologie 13). Hogrefe, Göttingen 2010

Horgan AF, Prasad B, Waldron DJ, O'Sullivan (1992): Acute Urinary Retention. Comparison of Suprapubic and Urethral Catheterisation. Br J Urol 므, 149-151

Ichsan J, Hunt DR (1987): Suprapubic catheters: A comparison of suprapubic versus urethral catheters in the treatment of acute urinary retention. Aust N Z J Surg $\underline{57,33-}$ 36

Krieger JN, Nyberg L, Nickel JC (1999): NIH consensus definition and classification of prostatitis. JAMA $\underline{281(3)}, 236-237$

Librero J, Peiró S, Ordiñana R (1999): Chronic comorbidity and outcomes of hospital care: Length of stay, mortality, and readmission at 30 and 365 days. J Clin Epidemiol 52, $171-179$ 
Lippert H: Lehrbuch Anatomie. 8. Auflage; Elsevier Urban \& Fischer, München 2011

Ludwig G, Haselberger J (1977): Epididymitis und Fertilität. Behandlungsergebnisse bei akuter unspezifischer Epididymitis. Fortschr Med $\underline{95}$, 397-399

Ludwig G: Infektionen des äußeren Genitale. In: Schmelz HU, Sparwasser C, Weidner W (Hrsg.): Facharztwissen Urologie. 2. Auflage; Springer, Heidelberg 2010a, 91 102

Ludwig G: Prostatitissyndrom und Vesikulitis. In: Schmelz HU, Sparwasser C, Weidner W (Hrsg.): Facharztwissen Urologie. 2. Auflage; Springer, Heidelberg 2010b,103112

Margreiter M, Shariat S: Prostatitis. In: Michel MS, Thüroff JW, Janetschek G, Wirth M (Hrsg.): Die Urologie. Band 2; Springer, Berlin Heidelberg 2016, 1295-1300

Merkle W: Entzündungen. In: Merkle W (Hrsg.): Urologie. Hippokrates-Verlag, Stuttgart 1997,116-148

Miettinen O (1974): Confounding and effect-modification. Am J Epidemiol 100, 350353

Muneishi R, Tanimoto R, Wada K, Hsiao P, Eguchi J, Araki M, Watanabe T, Nasu Y, Akebi N (2016): The gender specific risk factors for prolonged hospitalization due to acute pyelonephritis in a Japanese tertiary emergency center. $\mathrm{J}$ Infect Chemother $\underline{22}$, 108-111

Niedersächsisches Landesgesundheitsamt: Antibiotika-Resistenz-Monitoring in Niedersachsen.

https://www.nlga.niedersachsen.de/infektionsschutz/armin resistenzentwicklung/anti biotika-resistenz-monitoring-in-niedersachsen-armin-19418.html Zugriff am 2. Juli 2018

Osegbe DN (1991): Testicular function after unilateral bacterial epididymo-orchitis. Eur Urol $\underline{19}$, 204-208

Pearson K (2009): X. On the criterion that a given system of deviations from the probable in the case of a correlated system of variables is such that it can be reasonably supposed to have arisen from random sampling. Philos Mag $\underline{50}, 157-175$

Petry SJ, Gillitzer R: Fieber durch pathologische Befunde des Harntraktes. In: Thüroff JW (Hrsg.): Urologische Differenzialdiagnosen. 2. Auflage; Thieme, Stuttgart New York 2009, 77-86

Röhrig B, Du Prel J-B, Blettner M (2009): Studiendesign in der medizinischen Forschung-Teil 2 der Serie zur Berwertung wissenschaftlicher Publikationen. Dtsch Arztebl Int 106 (11) , 184-189 
Rupp TJ, Leslie SW. Epididymitis. [Updated 2018 Oct 27]. In: StatPearls [Internet]. Treasure Island (FL). https://www.ncbi.nlm.nih.gov/books/NBK430814/\#article21220.s1 Zugriff am 19.01.2018

Saint S, Chenoweth CE (2003): Biofilms and catheter-associated urinary tract infections. Infect Dis Clin North Am 17 (2), 411-432

Schaeffer AJ, Datta NS, Fowler JE, Krieger JN, Litwin MS, Nadler RB, Nickel JC, Pontari MA, Shoskes DA, Zeitlin SI (2002): Overview summary statement. Diagnosis and management of chronic prostatitis/chronic pelvic pain syndrome (CP/CPPS). Urology $\underline{60}(6), 1-4$

Schuppe H-C, Pilatz A, Hossain H, Diemer T, Wagenlehner F, Weidner W (2017): Urogenital Infection as a Risk Factor for Male Infertility. Dtsch Arztebl Int 114, 339346

Schünke M, Schulte E, Schumacher U, Voll M, Wesker K: Innere Organe (Prometheus LernAtlas der Anatomie). 3. Auflage; Thieme, Stuttgart 2012

Schwarz J, Bruderer Enzler H: Wilxocon-Rangsummentest-

Methodenberatung_Zürich 2016a.

http://www.methodenberatung.uzh.ch/de/datenanalyse/unterschiede/zentral/wilkoxon .html Zugriff am 3.03.2018

Schwarz J, Bruderer Enzler H: Pearson Chi-Quadrat-Test-Methodenberatung Zürich 2016b.

https://www.methodenberatung.uzh.ch/de/datenanalyse spss/unterschiede/proportio nen/pearsonuntersch.html Zugriff am 03.03.2018

Secchi A, Ziegenfuß T: Checkliste Notfallmedizin. 4. vollst. überarb. Auflage; Thieme, Stuttgart 2009

SF-12-Fragebogen-Kurzversion des SF-36. http://www.methodenlehrebaukasten.de/web/html/quellen/sf-12.html, Zugriff am 12.12.17

SF-36-Fragebogen. https://www.testzentrale.de/shop/fragebogen-zumgesundheitszustand.html, abgerufen am: 12.12.17

Shapiro SS, Wilk MB (1965): An Analysis of Variance Test for Normality (Complete Samples). Biometrika $\underline{52}$ (3/4), 591-611

Sökeland J, Brühl P, Hertle L, Piechota H (2000): Katheterdrainage der Harnblase heute. Dtsch Arztebl Int 97 (4), 2000, 168-174

Spitzer WO, Dobson AJ, Hall J, Chesterman E, Levi J, Shepherd R, Battista RN, Catchlove BR (1981): Measuring the quality of life of cancer patients. J Chron Dis $\underline{34}$, 585-597 
Talan DA, Stamm WE, Hooton TM, Moran GJ, Burke T, Iravani A, Reuning-Scherer J, Church DA (2000): Comparison of ciprofloxacin (7 days) and trimethoprimsulfamethoxazole (14 days) for acute uncomplicated pyelonephritis pyelonephritis in women: A randomized trial. JAMA $\underline{283}$ (12), 1583-1590

Tarlov AR, Ware JE, Greenfield S, Nelson EC, Perrin E, Zubkoff M (1989): The Medical Outcomes Study. An application of methods for monitoring the results of medical care. JAMA 262 (7), 925-930

van der Linden MW, Westert GP, Bakker DH de, Schellevis FG (2004): Second National Study on diseases and operations in general practice. Complaints and disorders in the population and in general practice https://www.nivel.nl/sites/default/files/bestanden/ns2_rapport1.pdf Zugriff am 13.12.2017

Vahlensieck W: Pyelonephritis und Nierenabszess. In: Schmelz HU, Sparwasser C, Weidner W (Hrsg.): Facharztwissen Urologie. 2. Auflage; Springer, Heidelberg 2010,17-50

Valente MJ, Pelham WE, Smyth H, MacKinnon DP (2017): Confounding in statistical mediation analysis: What it is and how to address it. J Couns Psychol $\underline{64}$ (6), 659671

Ware J, Kosinski M, Keller SD (1996): A 12-Item Short-Form Health Survey: Construction of scales and preliminary tests of reliability and validity. Med Care $\underline{34}$ (3), 220-233

Warren JW (2001): Catheter-associated urinary tract infections. Int J Antimicrob Ag 17 (4), 299-303

Weidner W, Schiefer HG, Garbe C (1987): Acute nongonococcal epididymitis. Aetiological and therapeutic aspects. Drugs $\underline{34}(1), 111-117$

Weidner W, Jantos C, Schiefer HG, Haidl G, Friedrich HJ (1991a): Semen parameters in men with and without proven chronic prostatitis. Arch Androl 26 (3), 173-183

Weidner W, Schiefer HG, Jantos C, Krauss H, Friedrich HJ, Altmannsberger M (1991b): Chronic prostatitis: A thorough search for etiologically involved microorganisms in 1,461 patients. Infection 19 (3), 119-125

Wetherill GB: Regression analysis with applications (Monographs on statistics and applied probability 27). Chapman and Hall, London 1986

Wilbert DM: Genitale Schmerzen. In: Thüroff JW (Hrsg.) :Urologische Differenzialdiagnose. 2. Auflage; Thieme, Stuttgart New York 2009, 35-52

Wilcoxon F (1945): Individual Comparisons by Ranking Methods. Biometrics Bull1 (6), $80-83$ 
Yoon BI, Kim S, Han D-S, Ha U-S, Lee S-J, Kim HW, Han C-H, Cho Y-H (2012):

Acute bacterial prostatitis: How to prevent and manage chronic infection? J Infect Chemother 18(4), 444-450 


\section{Danksagung}

Mein ganz besonderer Dank gilt meinem Doktorvater Prof. Dr. med. Sascha Ahyai für die hervorragende Betreuung meiner Doktorarbeit. Seine Idee für die Fragestellung der Katheterintervention bildet die Grundlage für meine Dissertation. Dafür sowie für die stetige Ansprechbarkeit bin ich sehr dankbar.

Ein weiterer großer Dank geht an meinen Betreuer Dr. med. Oliver Hahn für die fantastische Betreuung meiner Arbeit. Vielen Dank für die zahlreichen spontanen Treffen trotz der vielen Dienste, für das große Engagement sowie für die Korrekturen meiner Arbeit.

Außerdem danke ich Dr. med. Annemarie Uhlig für ihre Unterstützung und Hilfestellungen bei der statistischen Auswertung dieser Arbeit. 